\title{
The Progress of Cobalt-Based Anode Materials for Lithium Ion Batteries and Sodium Ion Batteries
}

\author{
Yaohui Zhang ${ }^{1, *}$, Nana Wang ${ }^{2}$ and Zhongchao Bai ${ }^{2, *}$ \\ 1 School of Energy and Power Engineering, North University of China, Taiyuan 030051, China \\ 2 College of Sciences, Henan Agricultural University, Zhengzhou 450002, China \\ * Correspondence: zhangyaohui@nuc.edu.cn (Y.Z.); baizhongchao@tyut.edu.cn (Z.B.)
}

Received: 26 March 2020; Accepted: 20 April 2020; Published: 29 April 2020

\begin{abstract}
Limited by the development of energy storage technology, the utilization ratio of renewable energy is still at a low level. Lithium/sodium ion batteries (LIBs/SIBs) with high-performance electrochemical performances, such as large-scale energy storage, low costs and high security, are expected to improve the above situation. Currently, developing anode materials with better electrochemical performances is the main obstacle to the development of LIBs/SIBs. Recently, a variety of studies have focused on cobalt-based anode materials applied for LIBs/SIBs, owing to their high theoretical specific capacity. This review systematically summarizes the recent status of cobalt-based anode materials in LIBs/SIBs, including $\mathrm{Li}^{+} / \mathrm{Na}^{+}$storage mechanisms, preparation methods, applications and strategies to improve the electrochemical performance of cobalt-based anode materials. Furthermore, the current challenges and prospects are also discussed in this review. Benefitting from these results, cobalt-based materials can be the next-generation anode for LIBs/SIBs.
\end{abstract}

Keywords: cobalt-based materials; anode; lithium ion battery; sodium ion battery

\section{Introduction}

With the increasing consumption of fossil energy, more and more attention has been paid to the development and utilization of renewable energy. However, new energy resources like wind, solar and tidal power are not sustainable by virtue of their uncertainty over time. In this scenario, the development of high-performance energy storage devices is highly necessary. To this end, rechargeable batteries (RBs) are of particular interest due to the multiple advantages of rational working voltage, high capacities and long cycling life [1-4]. Lithium ion batteries (LIBs), as one of the dominant RBs, have gradually penetrated into many aspects of our lives, such as portable electronic devices [5], consumable electronics [6] and electric vehicles [7]. However, the large-scale application of LIBs is hobbled by the rareness of lithium resources in the earth's crust [8,9]. Sodium ion batteries (SIBs) have captured great concerns as a complementary technology for LIBs by virtue of abundant raw materials. Furthermore, many electrode materials of LIBs can be applied as a drop-in replacement for SIBs because of a similar conversion reaction mechanism with LIBs.

There is a growing demand for the energy density of RBs. For LIBs, the mainstream anode materials-graphite materials are unable to meet the ever-increasing requirement of energy density because of the relatively lower theoretical specific capacity $\left(\sim 372 \mathrm{~mA} \mathrm{~h} \mathrm{~g}^{-1}\right)$. As for SIBs, the larger radius of $\mathrm{Na}^{+}\left(1.02 \AA\right.$ vs. $\left.0.76 \AA \mathrm{Af}^{+}\right)$gives rise to severe volume fluctuations of electrode materials and sluggish diffusion kinetics, finally yielding poor electrochemical performances of SIBs. Up to now, there have been no right anode materials of SIBs for practical applications [10]. Therefore, a key requirement for both LIBs and SIBs is the development of anode materials with applicable working voltage, marvelous cycle performance and high energy density. 
Up to now, unceasing efforts have been devoted to exploring appropriate anode materials, like metal chalcogenides, alloying metals, metal phosphide and carbon materials. Among these materials, cobalt-based composites (oxides, sulfides, phosphides and alloys) have recently drawn enormous attention owing to their outstanding electrochemical performances. For example, Zhang et al. synthesized a porous fiber structured $\mathrm{Co}_{3} \mathrm{O}_{4} @$ carbon for LIBs that delivered a capacity of $558 \mathrm{~mA} \mathrm{~h}$ $\mathrm{g}^{-1}$ after 500 cycles at a current density of $5 \mathrm{~A} \mathrm{~g}^{-1}$ [11]. Bai's group fabricated a spongy $\mathrm{CoS}_{2} /$ carbon composite that showed marvelous electrochemical performances as anode materials for both LIBs and SIBs. It retained a capacity of $610 \mathrm{~mA} \mathrm{~h} \mathrm{~g}^{-1}$ after 120 cycles at $0.5 \mathrm{~A} \mathrm{~g}^{-1}$ in LIBs and $330 \mathrm{~mA}$ $\mathrm{h} \mathrm{g}^{-1}$ after 60 cycles at $0.5 \mathrm{~A} \mathrm{~g}^{-1}$ in SIBs, respectively [12]. Zhang and his colleagues synthesized $\mathrm{CoP}$ nanoparticles embedded in $\mathrm{N}$-doped carbon nanosheets that displayed a Na-storage capacity of $598 \mathrm{~mA} \mathrm{~h} \mathrm{~g}^{-1}$ at $0.1 \mathrm{~A} \mathrm{~g}^{-1}$ [13].

Nonetheless, a large proportion of cobalt-based electrode materials are subjected to severe volume fluctuations caused by the uptaking and releasing of $\mathrm{Li}^{+}$or $\mathrm{Na}^{+}$, which eventually yield a pulverization of electrode materials and detachment from the current collector, finally giving rise to a decaying of capacity [14-16]. To circumvent these issues, plenty of cobalt-based materials with special structures have been reported [17-20]. In general, active materials with a nanometer scale can be conducive to the transfer of $\mathrm{Li}^{+}$or $\mathrm{Na}^{+}$owing to shorter diffusion lengths and tolerate the strain caused by volume expansion during periodical cycle processes, consequently enhancing electrochemical performances [21,22]. Zhang et al. fabricated $\mathrm{Co}_{9} \mathrm{~S}_{8} @$ carbon nanospheres with an average size of $60 \mathrm{~nm}$, which delivers an astonishing rate capability of $305 \mathrm{~mA} \mathrm{~h} \mathrm{~g}^{-1}$ after 1000 cycles at $5 \mathrm{~A} \mathrm{~g}^{-1}$ for anode materials of SIBs [23]. $\mathrm{CoSe}_{2} / \mathrm{N}$-doped carbon nanofibers synthesized by Li's group shows excellent structure retention. Even cycled for 1000 cycles, the sample well maintained its original structure without pulverization or amorphization [24].

Furthermore, the electrochemical properties of cobalt-based materials can also be improved by combining with carbonaceous buffers $[19,25,26]$. Beneficially, the carbonaceous buffer can enhance the electrical conductivity of the composite, as well as mitigate pulverization and aggregation during periodical cycles. Ge et al. synthesized unique CoP@C-reduced graphene oxide-nickel foam composite as the anode for SIBs that delivered a splendid capacity of $473.1 \mathrm{~mA} \mathrm{~h} \mathrm{~g}^{-1}$ at $0.1 \mathrm{~A} \mathrm{~g}^{-1}$ after 100 cycles [27]. $\mathrm{Co}_{9} \mathrm{~S}_{8}$ embedded in N-rich carbon hollow spheres fabricated by Fang's group exhibited a discharge capacity of $518 \mathrm{~mA} \mathrm{~h} \mathrm{~g}^{-1}$ at a current density of $2.176 \mathrm{~A} \mathrm{~g}^{-1}$ for LIBs [28]. To carefully study the origin behind the marvelous storage properties, many advanced testing technologies like in-situ X-ray diffraction, in-situ Nyquist plots, in-situ transmission electron microscopy and first-principles calculations are applied to discover the $\mathrm{Li} / \mathrm{Na}$-ion storage mechanism in cobalt-based electrode materials containing morphological evolution, phase transformation, kinetics, etc. These findings will construct a theoretical basis for a more applicable design of cobalt-based materials as an anode for LIBs and SIBs [29-33].

Until now, a lot of reports on cobalt-based electrode materials have been published. However, there is no review focused on a cobalt-based anode for LIBs/SIBs. In this review, we summarized the recent development of cobalt-based anode materials for LIBs and SIBs, including electrochemical mechanisms, synthetic strategies and common applications. Moreover, we also remarked on the future prospects of cobalt-based anode materials within the rapidly developing energy storage field.

\section{2. $\mathrm{Li}^{+} / \mathrm{Na}^{+}$Storage Mechanism}

$\mathrm{Li}^{+} / \mathrm{Na}^{+}$storage mechanisms for different Co-based materials are shown in Figure 1. Co-based electrode materials can be roughly divided into three types: Co-based alloys [19,34]; compounds of cobalt and nonmetals $\left(\mathrm{Co}_{x} \mathrm{~A}_{y}, \mathrm{~A}=\mathrm{O}, \mathrm{P}\right.$ and $\mathrm{S} \ldots$... $)$ [18,35-38] and other composites fabricated by cobalt and metal nonmetals [39]. Unlike graphite-based materials, $\mathrm{Li}^{+} / \mathrm{Na}^{+}$storage mechanisms of cobalt-based electrode materials consist mainly of alloying and conversion reactions. During the processes of alloying and conversion reactions, atomic rearrangements occur along with structural evolution and phase transformation, thereby yielding severe volume fluctuations during battery cycles. 


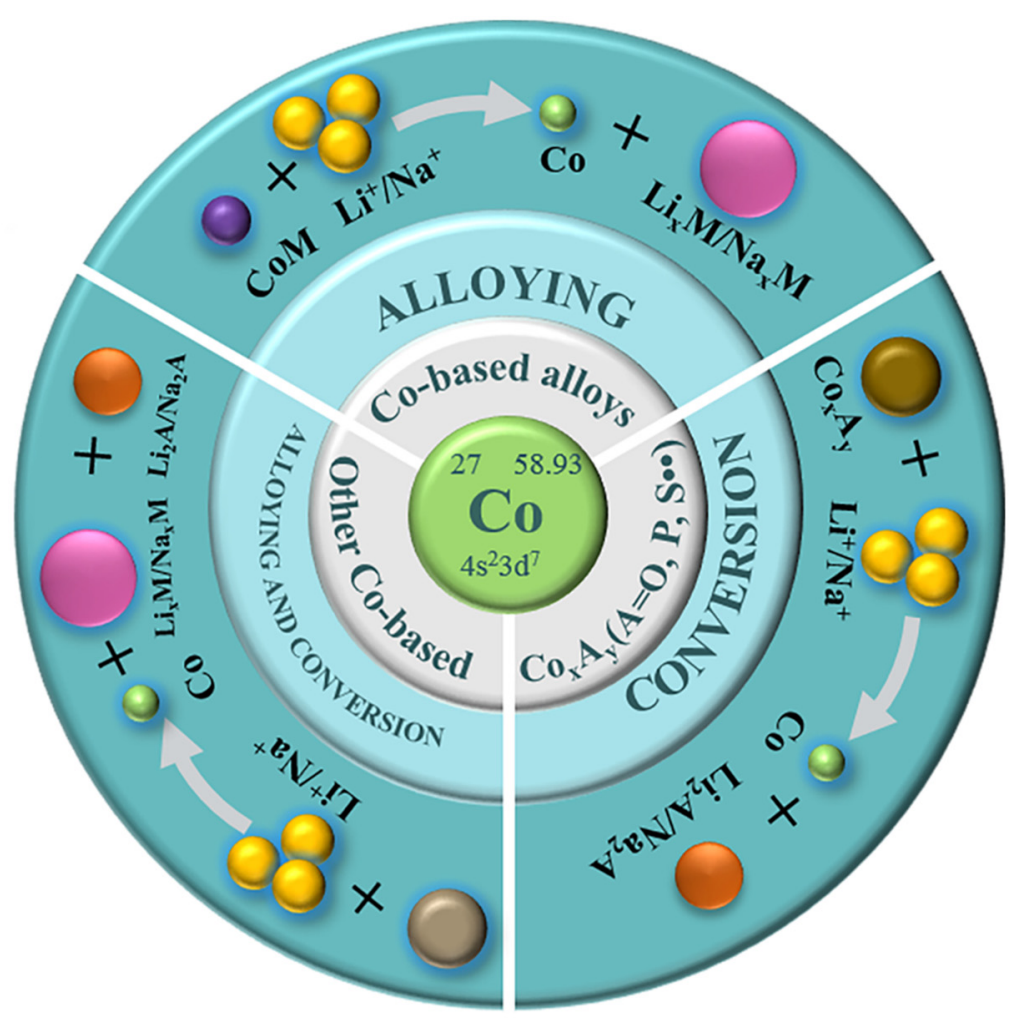

Figure 1. $\mathrm{Li}^{+} / \mathrm{Na}^{+}$storage mechanisms for different Co-based materials.

\section{1. $\mathrm{Li}^{+} / \mathrm{Na}^{+}$Storage Mechanism in Co-Based Alloys}

For cobalt-based alloys, lithium/sodium ion storage properties are decided by metal components that make up the alloy with cobalt [40-43]. Even so, the presence of cobalt components in the alloys is essential, which disperses into the alloys to improve the conductivity of alloys, as well as the retard volume expansion during cycles [44,45]. Many types of research on alloying storage mechanisms of cobalt-based materials have been published. For example, Yu et al. briefly pointed out the lithium ion storage mechanisms of Sn-Co@polymethylmethacrylate (PMMA) nanospheres [40]. In Figure 2a, an obvious slope between 1.2 and $0.4 \mathrm{~V}$ during the first discharge can be ascribed to the formation of SEI film. Then, a plat plateau at $0.3 \mathrm{~V}$ is attributed to the initial lithiation of the Sn component of the alloy, including the isolation of $\mathrm{Co}$ and the formation of $\mathrm{Li}_{\mathrm{x}} \mathrm{Sn}$. Subsequently, the platform centered at about $0.5 \mathrm{~V}$ during the charge can be ascribed to the delithiation of $\mathrm{Li}_{x} \mathrm{Sn}$ and the formation of $\mathrm{Sn}$. The entire lithium storage process of the alloy can be described by following equations:

$$
\begin{aligned}
\mathrm{Co}_{y} \mathrm{Sn}+\mathrm{xLi}^{+}+\mathrm{xe}^{-} & \rightarrow \mathrm{Li}_{x} \mathrm{Sn}+\mathrm{yCo}(0<\mathrm{x}<4.4) \text { Discharge } \\
\mathrm{Li}_{x} \mathrm{Sn}+\mathrm{yCo} & \rightarrow \mathrm{Sn}+\mathrm{yCo}+\mathrm{xLi}^{+}+\mathrm{xe}^{-} \text {Charge }
\end{aligned}
$$



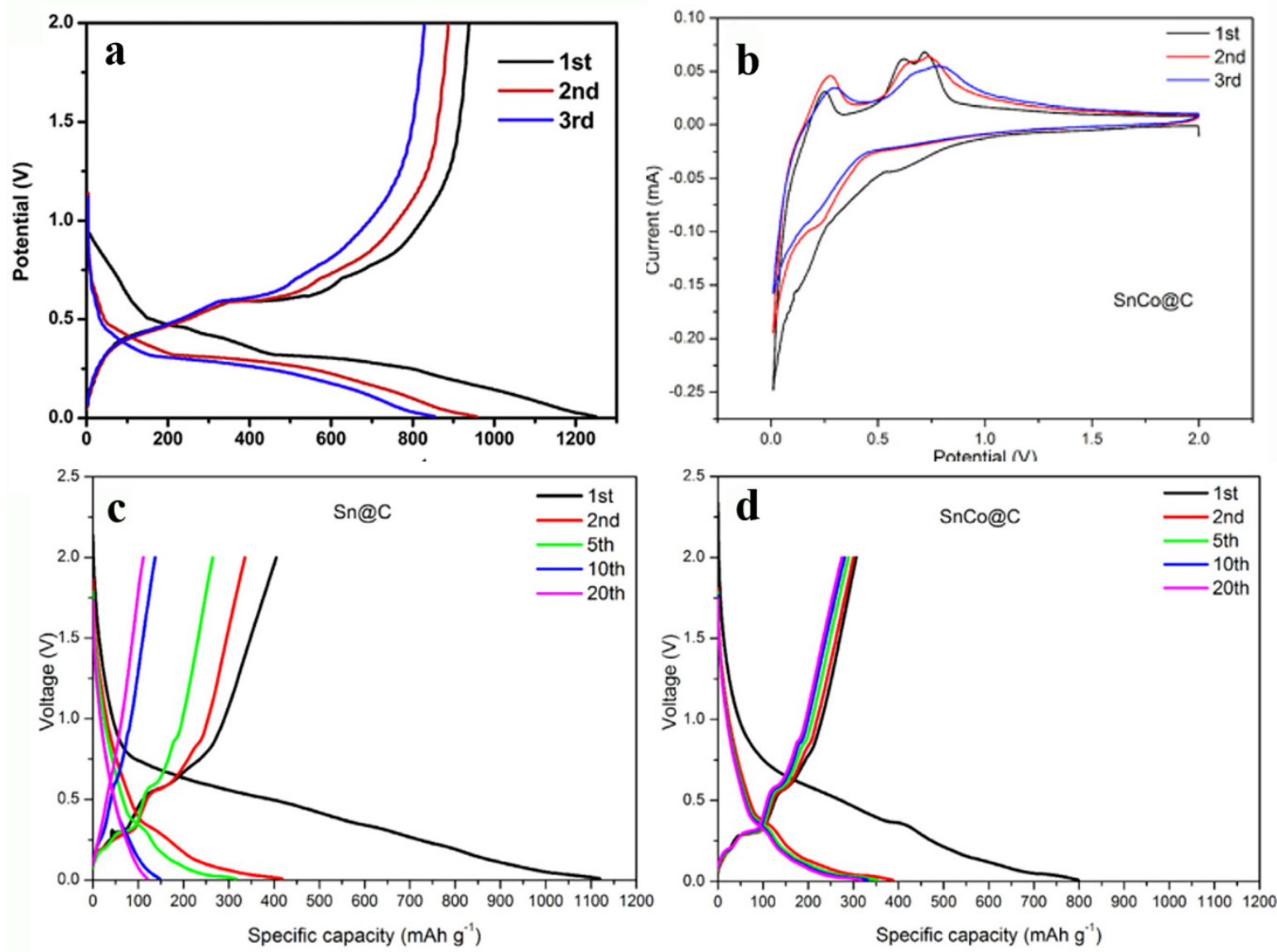

Figure 2. $\mathrm{Li}^{+}$and $\mathrm{Na}^{+}$storage in the Sn-Co alloy anode. (a) Charge-discharge profiles of Sn-Co@polymethyl methacrylate (PMMA) for the initial three cycles in lithium ion batteries (LIBs) [40]. (b) Cyclic voltammetry (CV) curves of SnCo@C in sodium ion batteries (SIBs) for the initial three cycles [41]. (c) and (d) Charge-discharge curves of Sn@C and SnCo@C at different cycles in SIBs, respectively [41].

As displayed in Figure $2 b$, the sodium storage mechanism of cobalt alloy can be simply demonstrated by cyclic voltammetry (CV) curves of SnCo@C in SIBs for the initial three cycles [41]. During the first discharge process, peaks centered at around 0.75 and $0.15 \mathrm{~V}$ can be ascribed to the first sodiation of the alloy, which indicated the formation of $\mathrm{Na}_{x} \mathrm{Sn}$ and Co. The peaks at 0.3, 0.6 and $0.7 \mathrm{~V}$ upon the charge process represented a desodiation process from $\mathrm{Na}_{x} \mathrm{Sn}$ to $\mathrm{Sn}$. Similar to the lithium storage mechanism, the sodium storage mechanism of SnCo@C can be concluded by following equations:

$$
\begin{gathered}
\mathrm{SnCo}+\mathrm{xNa}^{+}+\mathrm{xe}^{-} \rightarrow \mathrm{Na}_{x} \mathrm{Sn}+\mathrm{Co}(0<\mathrm{x}<4.4) \text { Discharge } \\
\mathrm{Na}_{x} \mathrm{Sn}+\mathrm{Co} \rightarrow \mathrm{Sn}+\mathrm{Co}+\mathrm{xNa}^{+}+\mathrm{xe}^{-} \text {Charge }
\end{gathered}
$$

In Figure 2c,d, the charge-discharge profiles of Sn@C and SnCo@C displayed similar voltage platforms at different cycles, indicating the decisive role of the metal component alloyed with cobalt. Furthermore, the presence of cobalt improves the capacity retention ratio during cycles, suggesting the importance of cobalt.

\section{2. $\mathrm{Li}^{+} / \mathrm{Na}^{+}$Storage Mechanism in $\mathrm{Co}_{x} A_{y}(A=\mathrm{O}, \mathrm{S}, \mathrm{P}$ and $\mathrm{Se})$}

$\mathrm{Co}_{\mathrm{x}} \mathrm{A}_{\mathrm{y}}$ electrode materials consist of cobaltous oxides, cobaltous sulfides, cobaltous phosphide and cobaltous selenide. $\mathrm{Li}^{+} / \mathrm{Na}^{+}$storage mechanism in $\mathrm{Co}_{x} \mathrm{~A}_{y}$ is the conversion reaction that eventually 
forms $\mathrm{Co}$ and $\mathrm{Li}_{x} \mathrm{~A}$ or $\mathrm{Na}_{x} \mathrm{~A}[14,15,20,27,35,46-48]$. The typical reactions of $\mathrm{Co}_{x} \mathrm{~A}_{\mathrm{y}}$ with $\mathrm{Li}^{+}$is displayed in Table 1. It should be noted that some temporary intermediate reactions may occur during the discharge process of $\mathrm{Co}_{\mathrm{x}} \mathrm{A}_{\mathrm{y}}$, as depicted in the following equations:

$$
\mathrm{Co}_{x} \mathrm{~A}_{y}+\mathrm{Li}^{+}+\mathrm{e}^{-} \rightarrow \mathrm{LiCo}_{x} \mathrm{~A}_{y}
$$

or

$$
\mathrm{Co}_{x} \mathrm{~A}_{\mathrm{y}}+\mathrm{Li}^{+}+\mathrm{e}^{-} \rightarrow \mathrm{Co}_{\mathrm{x}} \mathrm{A}_{\mathrm{y}-1}+\mathrm{LiA}
$$

Table 1. $\mathrm{Li}^{+}$storage mechanism of some $\mathrm{Co}_{x} \mathrm{~A}_{y}$-active materials.

\begin{tabular}{ccc}
\hline Active Materials & $\mathrm{Li}^{+}$Storage Mechanism & Reference \\
\hline $\mathrm{Co}_{3} \mathrm{O}_{4}$ & $\mathrm{Co}_{3} \mathrm{O}_{4}+8 \mathrm{Li}^{+}+8 \mathrm{e}^{-} \rightarrow 3 \mathrm{Co}+4 \mathrm{Li}_{2} \mathrm{O}$ & {$[15]$} \\
$\mathrm{Co}_{2} \mathrm{P}$ & $\mathrm{Co}_{2} \mathrm{P}+3 \mathrm{Li}^{+}+3 \mathrm{e}^{-} \rightarrow 2 \mathrm{Co}+\mathrm{Li}_{3} \mathrm{P}$ & {$[46]$} \\
$\mathrm{CoS}_{2}$ & $\mathrm{CoS}_{2}+4 \mathrm{Li}^{+}+4 \mathrm{e}^{-} \rightarrow \mathrm{Co}+2 \mathrm{Li}_{2} \mathrm{~S}$ & {$[14]$} \\
$\mathrm{CoSe}_{2}$ & $\mathrm{CoSe}_{2}+4 \mathrm{Li}^{+}+4 \mathrm{e}^{-} \rightarrow \mathrm{Co}+2 \mathrm{Li}_{2} \mathrm{Se}$ & {$[20]$} \\
\hline
\end{tabular}

Furthermore, the sodium storage reactions are similar to lithium in terms of the conversion reaction accompanied by temporary intermediate reactions. Zhang's group (Figure 3a) investigated the sodium storage behavior of shale-like $\mathrm{Co}_{3} \mathrm{O}_{4}$ by cyclic voltammetry (CV) from 0.005 to $2.9 \mathrm{~V}$ at $0.1 \mathrm{mV} / \mathrm{s}$ [35]. During the first discharge process, they found an intermediate reaction initially happened at $0.76 \mathrm{~V}$, with $\mathrm{Co}_{3} \mathrm{O}_{4}$ reduced to $\mathrm{CoO}$ and $\mathrm{Na}_{2} \mathrm{O}$, provisionally. Subsequently, the resulting $\mathrm{CoO}$ intermediate phase was further converted $(0.39 \mathrm{~V})$ to metallic $\mathrm{Co}$ and $\mathrm{Na}_{2} \mathrm{O}$. Upon oxidation, metallic Co was step-by-step oxidized from $\mathrm{CoO}$ to $\mathrm{Co}_{3} \mathrm{O}_{4}$ with the extraction of $\mathrm{Na}^{+}$from $\mathrm{Na}_{2} \mathrm{O}$. Two main anodic peaks centered at 0.88 and $1.65 \mathrm{~V}$ corresponding to the reversible formation of $\mathrm{CoO}$ and $\mathrm{Co}_{3} \mathrm{O}_{4}$, respectively. The sodium storage reactions of the shale-like $\mathrm{Co}_{3} \mathrm{O}_{4}$ are as follows:

$$
\begin{gathered}
\mathrm{Co}_{3} \mathrm{O}_{4}+2 \mathrm{Na}^{+}+2 \mathrm{e}^{-} \rightarrow 3 \mathrm{CoO}+\mathrm{Na}_{2} \mathrm{O} \\
3 \mathrm{CoO}+6 \mathrm{Na}^{+}+6 \mathrm{e}^{-} \rightarrow 3 \mathrm{Co}+3 \mathrm{Na}_{2} \mathrm{O}
\end{gathered}
$$

Ge et al. investigated the $\mathrm{Na}^{+}$storage mechanism of CoP by using ex-situ TEM and CV tests [27]. In Figure 3b, two peaks centered at 0.2 and $0.05 \mathrm{~V}$ can be attributed to the stepwise formation of $\mathrm{Na}_{x} \mathrm{P}$ and $\mathrm{Na}_{3} \mathrm{P}$ during the first cathodic scan. During the subsequent charge process, a stepwise extraction of $\mathrm{Na}^{+}$occurred along with the decomposition of $\mathrm{Na}_{3} \mathrm{P}$, characterized by two anodic peaks located at 0.5 and 1.5 V. It should be noted that a partial $\mathrm{Na}_{3} \mathrm{P}$ transformed to $\mathrm{P}$ during the decomposition of $\mathrm{Na}_{3} \mathrm{P}$. The corresponding sodiation/desodiation reactions are as follows:

$$
\begin{gathered}
\mathrm{CoP}+3 \mathrm{Na}^{+}+3 \mathrm{e}^{-} \rightarrow \mathrm{Co}+\mathrm{Na}_{3} \mathrm{P} \\
\mathrm{Na}_{3} \mathrm{P} \rightarrow \mathrm{P}+3 \mathrm{Na}^{+}+3 \mathrm{e}^{-} \\
\mathrm{P}+3 \mathrm{Na}^{+}+3 \mathrm{e}^{-} \leftrightarrow \mathrm{Na}_{3} \mathrm{P}
\end{gathered}
$$

Fei and coworkers investigated the electrochemical reactions of $\mathrm{CoS}_{2} / \mathrm{rGO}$ by $\mathrm{CV}$ curves of the initial two cycles (Figure 3c) [49]. The peak located at $1.4 \mathrm{~V}$ can be ascribed to the intercalation of $\mathrm{Na}^{+}$into the $\mathrm{CoS}_{2}$ crystals in the first discharge process. The resulting intermediate $\mathrm{Na}_{\mathrm{x}} \mathrm{CoS}_{2}$ suffered a further conversion reaction to form $\mathrm{Na}_{2} \mathrm{~S}$ and metallic $\mathrm{Co}$ at $0.8 \mathrm{~V}$. Upon desodiation, two main oxidation peaks at 1.6 and $2.2 \mathrm{~V}$ can be due to the reversible formation of $\mathrm{Na}_{\mathrm{x}} \mathrm{CoS}_{2}$ and $\mathrm{CoS}_{2}$, respectively. The corresponding electrochemical reactions are as follows: 


$$
\begin{gathered}
\mathrm{CoS}_{2}+\mathrm{xNa}^{+}+\mathrm{xe}^{-} \leftrightarrow \mathrm{Na}_{\mathrm{x}} \mathrm{CoS}_{2} \\
\mathrm{Na}_{\mathrm{x}} \mathrm{CoS}_{2}+(4-\mathrm{x}) \mathrm{Na}^{+}+(4-\mathrm{x}) \mathrm{e}^{-} \leftrightarrow \mathrm{Co}+2 \mathrm{Na}_{2} \mathrm{~S}
\end{gathered}
$$
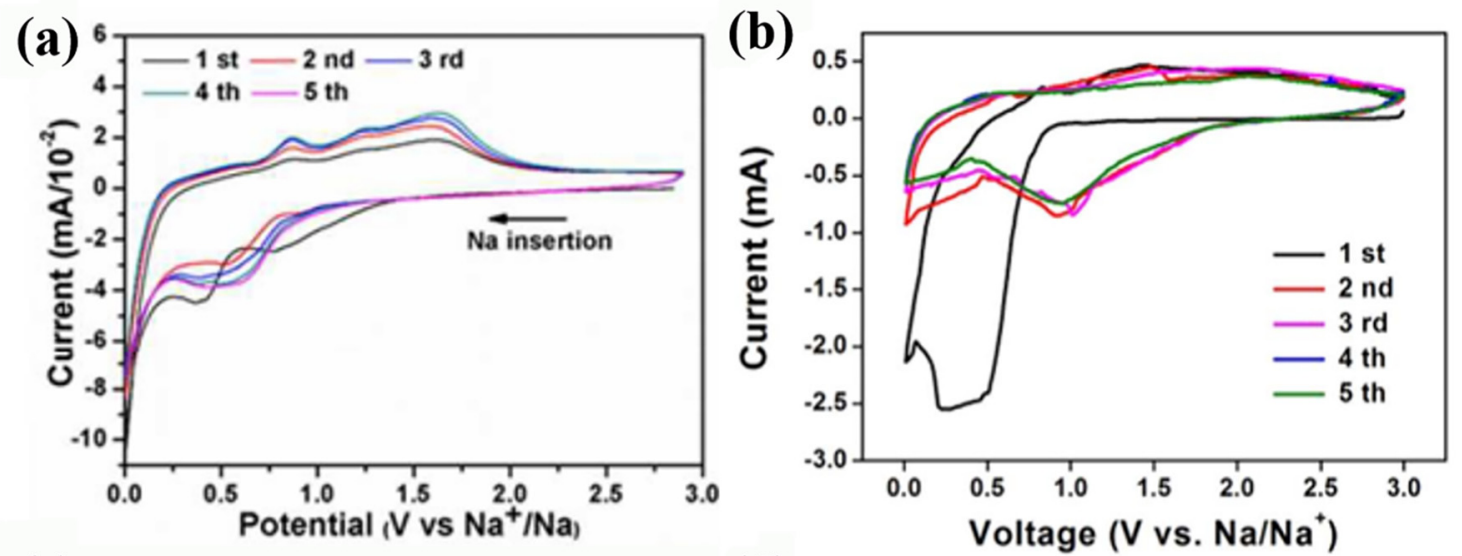

(c)

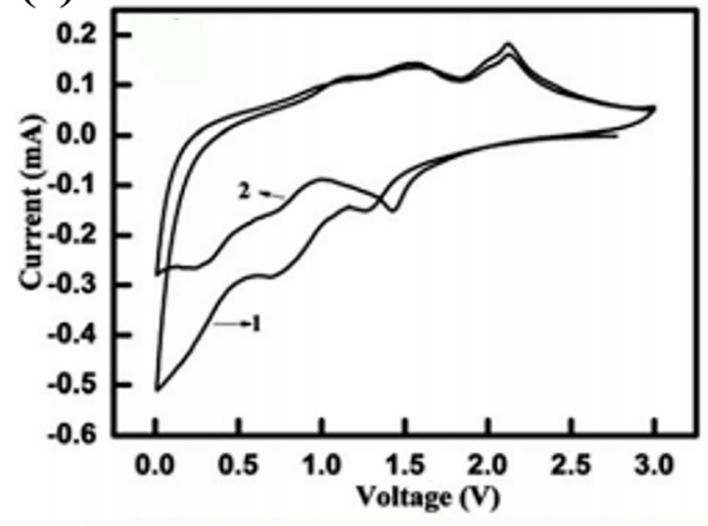

(d)

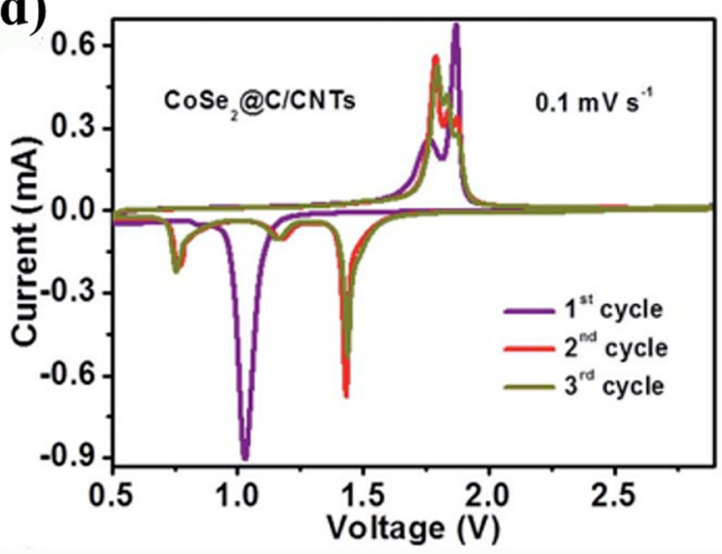

Figure 3. $\mathrm{CV}$ curves of (a) shale-like $\mathrm{Co}_{3} \mathrm{O}_{4}$ tested at $0.1 \mathrm{mV} / \mathrm{s}$ from 0.005 to $2.9 \mathrm{~V}$ vs. Na/Na+ [35]. (b) CoP@C-RGO-NF tested at $0.1 \mathrm{mV} / \mathrm{s}$ in the voltage range of $0.01-3 \mathrm{~V}$ vs. $\mathrm{Na} / \mathrm{Na}^{+}$[27]. (c) $\mathrm{CoS}_{2} / \mathrm{rGO}$ at a scan rate of $0.5 \mathrm{mV} / \mathrm{s}$ from $0.01-3 \mathrm{~V}$ vs. Na/Na ${ }^{+}$[49]. (d) $\mathrm{CoSe}_{2} @ \mathrm{C} /$ carbon nanotubes (CNTs) tested at $0.1 \mathrm{mV} / \mathrm{s}$ from $0.5-2.9 \mathrm{~V}$ [48].

The sodium storage reaction mechanism of $\mathrm{CoSe}_{2}$ was investigated by Qiu's group with a CV test, as shown in Figure 3d [48]. During the first anodic scan, two adjacent peaks at 1.7 and $1.87 \mathrm{~V}$ were attributed to stepwise conversion reactions from $\mathrm{Co}$ and $\mathrm{Na}_{2} \mathrm{Se}$ to an intermediate $\mathrm{Na}_{x} \mathrm{CoSe}$ and a final $\mathrm{CoSe}_{2}$, respectively. In the second anodic scan, three reduction peaks appeared at $1.4,1.1$ and $0.68 \mathrm{~V}$, corresponding to the uptake of $\mathrm{Na}^{+}$and a stepwise sodiation of $\mathrm{CoSe}_{2}$. The corresponding reactions can be seen as follows:

Discharge process:

$$
\begin{gathered}
\mathrm{CoSe}_{2}+\mathrm{xNa}^{+}+\mathrm{xe}^{-} \rightarrow \mathrm{Na}_{\mathrm{x}} \mathrm{CoSe}_{2} \\
\mathrm{Na}_{\mathrm{x}} \mathrm{CoSe}_{2}+(2-\mathrm{x}) \mathrm{Na}^{+}+(2-\mathrm{x}) \mathrm{e}^{-} \rightarrow \mathrm{CoSe}+\mathrm{Na}_{2} \mathrm{Se} \\
\mathrm{CoSe}+2 \mathrm{Na}^{+}+2 \mathrm{e}^{-} \rightarrow \mathrm{Co}+\mathrm{Na}_{2} \mathrm{Se}
\end{gathered}
$$

Charge process:

$$
\begin{gathered}
\mathrm{Co}+2 \mathrm{Na}_{2} \mathrm{Se} \rightarrow \mathrm{Na}_{x} \mathrm{CoSe}_{2}+(4-\mathrm{x}) \mathrm{Na}^{+}+(4-\mathrm{x}) \mathrm{e}^{-} \\
\mathrm{Na}_{x} \mathrm{CoSe}_{2} \rightarrow \mathrm{CoSe}_{2}+\mathrm{xNa}^{+}+\mathrm{xe}^{-}
\end{gathered}
$$


The $\mathrm{Li}^{+} / \mathrm{Na}^{+}$storage mechanism is decided by components that composited with cobalt. Similarly, other cobalt-based composites like $\mathrm{Co}_{2} \mathrm{SnO}_{4}, \mathrm{CoMn}_{2} \mathrm{O}_{4}$ and Sb-Co-P have more complex $\mathrm{Li}^{+} / \mathrm{Na}^{+}$ storage mechanisms, due to more components in composites [39,50-52]. Nevertheless, the $\mathrm{Li}^{+} / \mathrm{Na}^{+}$ storage mechanisms are still composed of basic alloying and conversion reactions.

\section{Preparation Methods of Synthesizing Cobalt-based Active Materials}

Up until now, many reports have been published for different synthetic strategies of various cobalt-based anode materials. In this section, we review some common methods.

\subsection{Hydrothermal/Solvothermal Methods}

As a typical synthetic method, hydrothermal/solvothermal reactions are mainly used to prepare materials by providing appropriate temperatures and times [53]. Under the circumstances of moderate temperatures and high pressures, various structured cobalt-based materials have been synthesized with high-phase purity and crystallinity. Moreover, hydrothermal/solvothermal methods are environmentally benign and cost-efficient due to their own characteristics [21,54]. Many cobalt-based anode materials with various morphologies have been synthesized by these methods, like nano-sized Co-Sn alloy, single-crystal intermetallic Co-Sn nanospheres and single-crystalline $\mathrm{Co}_{3} \mathrm{O}_{4}$ nanobelts [55-57].

Zhang's group synthesized a facile structure with $\mathrm{CoS}_{2}$ confined in the graphitic carbon walls of porous $\mathrm{N}$-doped carbon spheres ( $\mathrm{CoS}_{2}$-in-wall-NCSs) by the hydrothermal method associated with carbonization and sulfurization, as shown in Figure 4a-c [58]. During the process of hydrothermal, Co-containing melamine-phenolic resin spheres formed under the condition of high temperatures and high pressures. Followed with carbonization and sulfurization, $\mathrm{CoS}_{2}$ nanodots were evenly distributed throughout the carbon sphere. Figure $4 \mathrm{~d}$,e reveals the worm-like sample with $\mathrm{CuCo}_{2} \mathrm{~S}_{4}$ nanocrystalline (5-20 nm) evenly anchored on the carbon nanotubes (CNTs) synthesized by Jin et al. through solvothermal treatment [59]. CoSe@ carbon spheres (denoted as CoSe@CSs) were prepared through a facile solvothermal treatment by adding $\mathrm{Co}\left(\mathrm{NO}_{3}\right)_{2} \cdot 6 \mathrm{H}_{2} \mathrm{O}$ and $\mathrm{H}_{3} \mathrm{O}_{2} \mathrm{Se}$ to a moderate isopropanol solution [60]. The TEM image (Figure $4 \mathrm{f}$ ) reveals that CoSe nanoparticles uniformly embedded in amorphous carbon spheres $(100 \mathrm{~nm})$. Zhu's group synthesized a pure orthorhombic CoP film of nanorod arrays through a hydrothermal method [61]. As displayed in Figure 4g, these CoP nanorods were evenly distributed and interconnected with others to form a 3D interwoven. $\mathrm{Li}$ and coworkers reported a facile solvothermal reaction to synthesize $\mathrm{Co}_{3} \mathrm{O}_{4} / \mathrm{CNT}$ nanocomposites [62]. In Figure $4 \mathrm{~h}$, the cyboidal $\mathrm{Co}_{3} \mathrm{O}_{4}$ nanoparticles were evenly anchored on the surface of the CNT. The elemental mapping of the sample reveals the distribution of $\mathrm{C}, \mathrm{Co}$ and $\mathrm{O}$. 

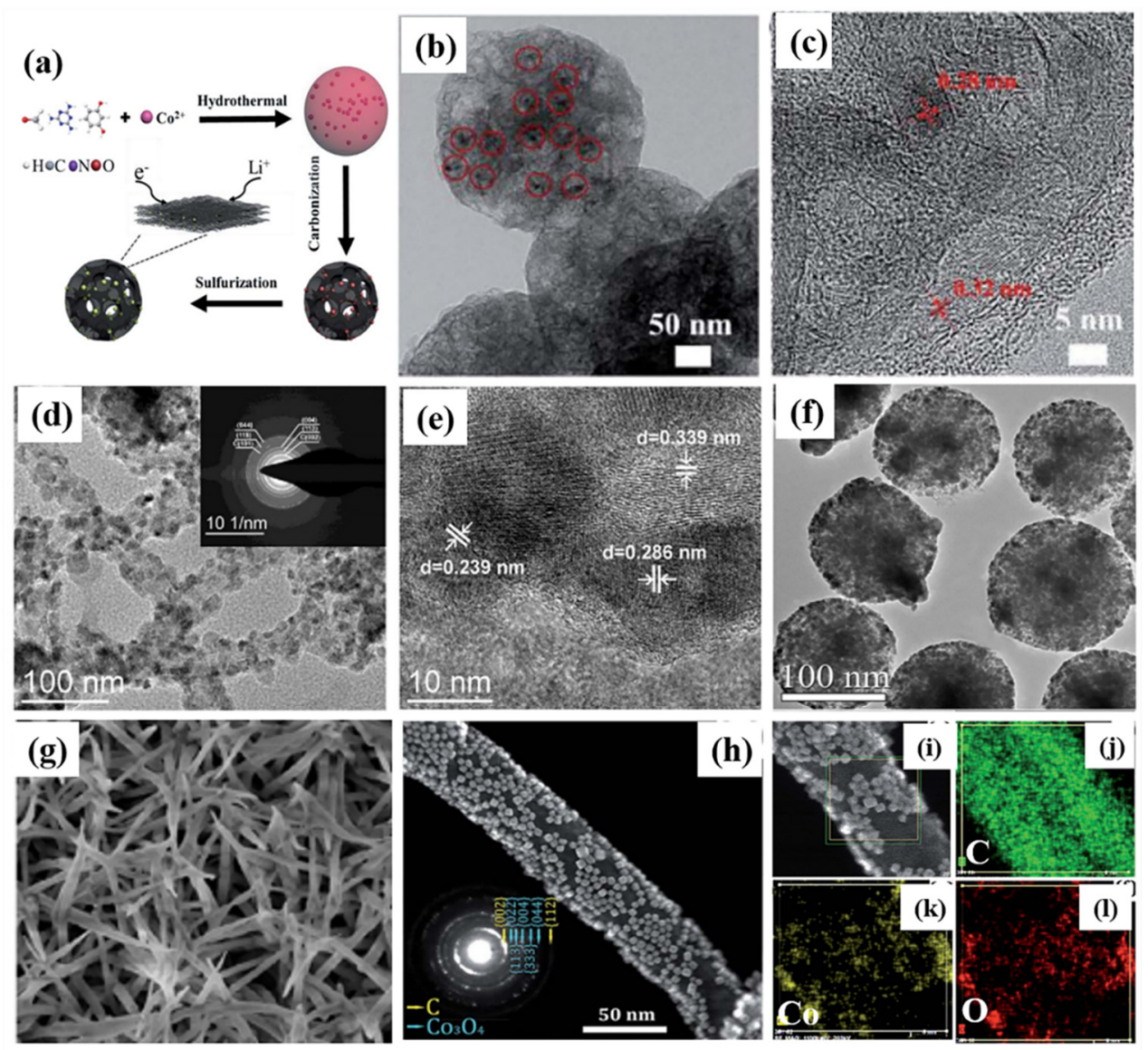

Figure 4. Preparation methods and morphology characterization of cobalt-based composites. (a) Schematic illustration of the preparation of (b) TEM and (c) HRTEM images of the $\mathrm{CoS}_{2}$-in-wall-N-doped carbon spheres (NCSs) [58]. (d) TEM image, SAED pattern (inset) and (e) HRTEM image of CNTs@CuCo $\mathrm{S}_{4}$ [59]. (f) Low-magnification TEM image of CoSe@100CSs [60]. (g) SEM image of CoP nanorod arrays [61]. (h) Low and (i) high-magnification SEM images and (i-k) elemental mapping images of $\mathrm{Co}_{3} \mathrm{O}_{4} / \mathrm{CNT}$ nanocomposites [62].

\subsection{Galvanic Replacement}

Galvanic replacement has been widely applied to synthesize nanostructured electrode materials due to its facile and low-temperature synthesis method. Zhou and coworkers synthesized a broccoli-like $\mathrm{Co}_{3} \mathrm{O}_{4} @ \mathrm{CNTs}$ anode material for SIBs through a galvanic replacement route [63]. Firstly, the acidizing operation of carbon nanotubes (CNTs) can create numerous functional groups with a negative charge on the surface of CNTs. Owing to electrostatic attraction, lots of $\mathrm{Co}^{2+}$ ions with a positive charge will attach to the surface of CNTs with carboxylic groups and serve as nucleation precursors. During the subsequent galvanic replacement reaction, a $\mathrm{Co}^{2+}$ ion was oxidized to $\mathrm{Co}_{3} \mathrm{O}_{4}$ at $140{ }^{\circ} \mathrm{C}$. After that, $\mathrm{Co}_{3} \mathrm{O}_{4}$ crystalline particles aggregated on the surface of CNTs owing to the electrostatic attraction and Van der Waals forces; thus, broccoli-like $\mathrm{Co}_{3} \mathrm{O}_{4} @ \mathrm{CNTs}$ were finally obtained (Figure 5a). The TEM and HRTEM images displayed in Figure 5b-d clearly confirmed that the well-crystallized $\mathrm{Co}_{3} \mathrm{O}_{4}$ nanocrystals were assembled on the surface of the CNTs. Ma and collogues successfully synthesized a novel Sn-Co@C alloy through galvanic replacement with metal-organic framework ZIF-67 as both the template and carbon source [64]. Figure 5e-g reveal that the Sn-Co nano-alloy particles $(\sim 10 \mathrm{~nm})$ were evenly embedded in porous $\mathrm{N}$-doped carbon, forming a novel hierarchical structure. In Figure $5 \mathrm{~h}-1$, the distribution of $\mathrm{C}, \mathrm{O}, \mathrm{N}, \mathrm{Co}$ and Sn elements clearly confirms the uniform structure of the Sn-Co@C alloy. 

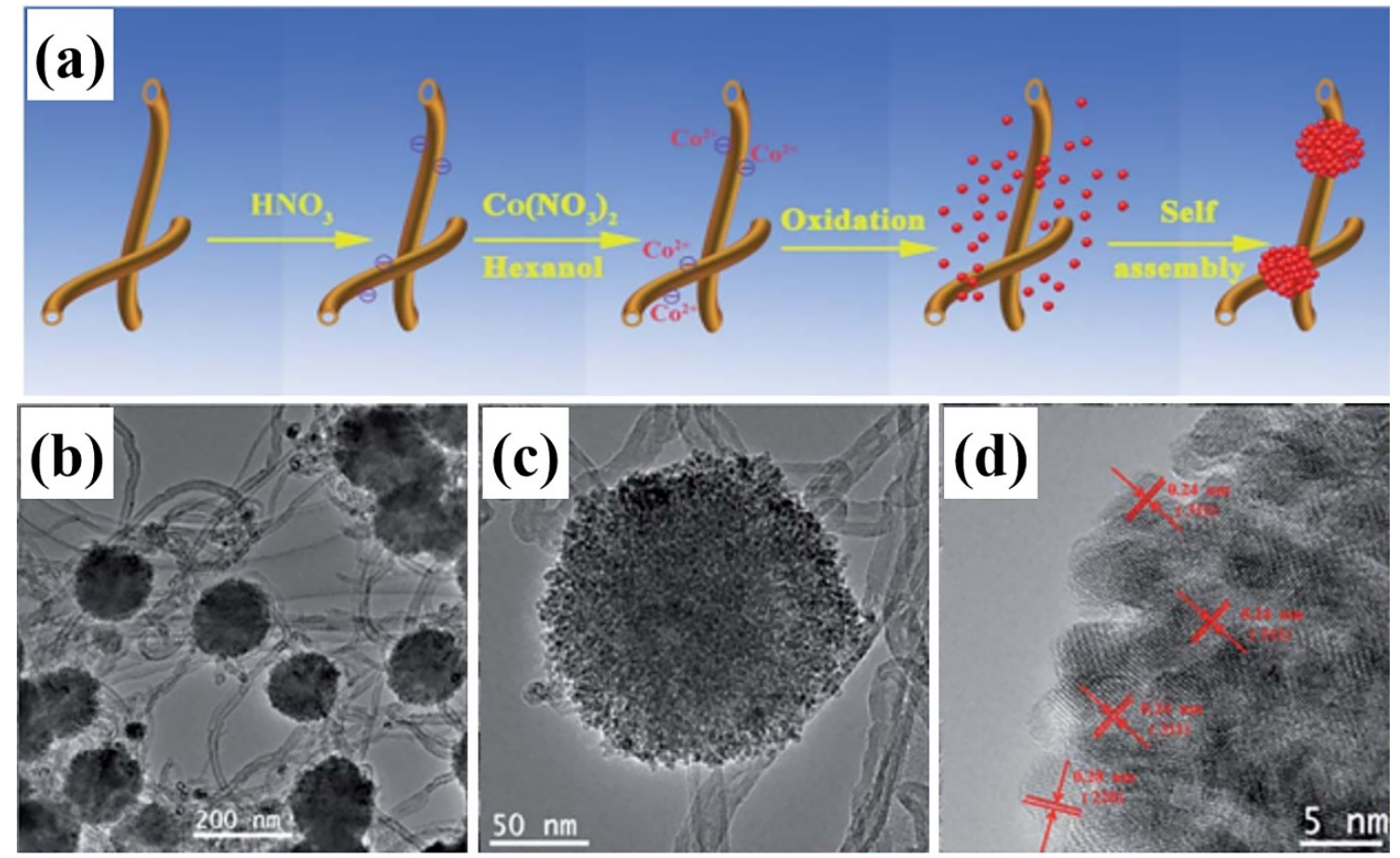
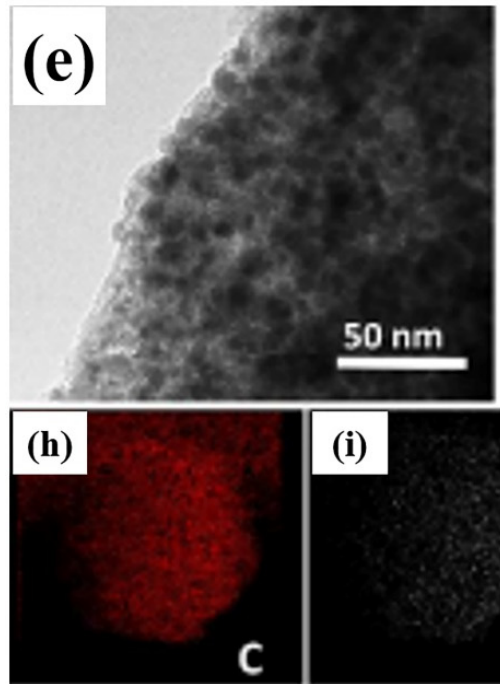

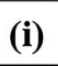

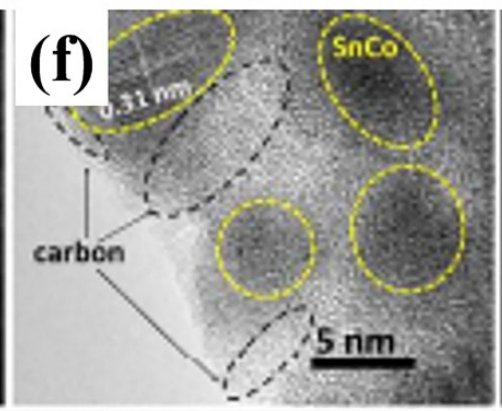

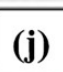

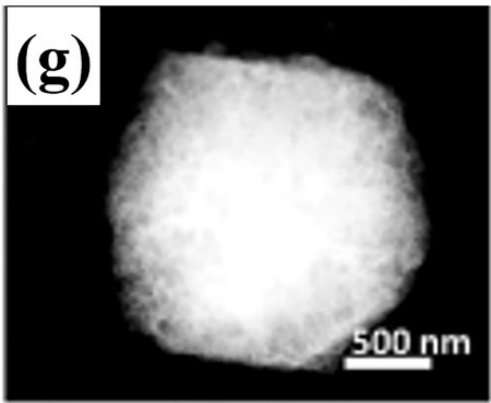

(I)

Figure 5. Morphology characterization of cobalt-based composites. (a) Schematic evolution of the $\mathrm{Co}_{3} \mathrm{O}_{4} @ \mathrm{CNT}$ composites. TEM images $(\mathbf{b}, \mathbf{c})$ and HRTEM image $(\mathbf{d})$ of the $\mathrm{Co}_{3} \mathrm{O}_{4} @ C N T$ composites [63]. TEM (e) and HRTEM (f) images of Sn-Co@C-2; STEM image (h) and corresponding elemental mapping images (h-1) of Sn-Co@C-2 [64].

Mesoporous $\mathrm{Co}_{3} \mathrm{O}_{4}$ nanowire arrays, needlelike $\mathrm{Co}_{3} \mathrm{O}_{4}$ nanotubes [65], porous carbon/ $\mathrm{CoS}_{2}$ [66] and some other cobalt-based anode materials with various morphologies and structures have also been synthesized by galvanic replacement [67].

\subsection{Heat Treatment}

As a high-temperature treatment, heat treatments can be used independently and combined with other synthesis methods, such as ball milling, electrostatic spinning and hydrothermal/solvothermal, to provide a special reaction condition for intermediate processes like sulfuration and carbonization. Lots of cobalt-based electrode materials with high performances have been successfully synthesized through heat treatments $[15,22,68-79]$. 
Pan's group successfully distributed CoSe nanoparticles into the porous carbon polyhedral (CoSe@PCP) through a two-step heat treatment [68]. During the first heat treatment, the precursor ZIF-67 was firstly converted to Co@PCP, including the carbonization of the organic linkers and reduction of the Co ion. Subsequently, CoSe@PCP was finally synthesized by selenization of Co@PCP at a high temperature (Figure 6a). As shown in Figure 6b, the resulting CoSe powders were uniformly embedded into porous carbon without aggregation. Zhang and coworkers prepared a 3D spongy $\mathrm{CoS}_{2} / \mathrm{C}$ anode material through a heating precursor accompanied by a hydrothermal process [12]. Figure $6 \mathrm{c}$ delivers a TEM image of the synthesized spongy $\mathrm{CoS}_{2} / \mathrm{C}$ with $\mathrm{CoS}_{2}$ nanoparticles $(\sim 20 \mathrm{~nm})$ homogeneously distributed in the porous carbon. Wang and colleagues realized accurate control over the synthesis of multi-shelled $\mathrm{Co}_{3} \mathrm{O}_{4}$ hollow microspheres by adjusting the solution and subsequent heating in the air [69]. The TEM and HRTEM images displayed in Figure 6d,e clearly reveal the morphology of triple-shelled $\mathrm{Co}_{3} \mathrm{O}_{4}$ hollow microspheres. Yin's group firstly synthesized a unique core-shell porous FeP@CoP phosphide microcube interconnected by reduced graphene oxide nanosheets (RGO@CoP@FeP) through a facile phosphorization process [70]. In Figure 6f, the SEM image of RGO@CoP@FeP reveals a typical hierarchical structure of wrinkled RGO interconnected microcubes. Qiu's group synthesized micro-scaled spherical $\mathrm{CoSn}_{2} / \mathrm{Sn}$ alloy through a sintering process, employing a stoichiometric amount of $\mathrm{SnO}_{2}, \mathrm{Co}_{3} \mathrm{O}_{4}$ and $\mathrm{C}$ as raw materials [22]. SEM observation of $\mathrm{CoSn}_{2} / \mathrm{Sn}$ alloy reveals that the particles were composed of lots of small grains, as shown in the inset of Figure $6 \mathrm{~g}$. Zeng et al. developed a facile heat treatment, composed of pyrolysis and sulfurization, to synthesize hollow $\mathrm{Co}_{9} \mathrm{~S}_{8} / \mathrm{N}-\mathrm{C}$ composites [28]. The SEM image of hollow $\mathrm{Co}_{9} \mathrm{~S}_{8} / \mathrm{N}-\mathrm{C}$ composites displayed in Figure $6 \mathrm{~h}$ reveals the thickness of the wall was approximately $70 \mathrm{~nm}$. $\mathrm{Co} /(\mathrm{NiCo}) \mathrm{Se}_{2}$ box-in-box structures were firstly fabricated by Kang's group via a selenization process at $\mathrm{Ar} / \mathrm{H}_{2}$, applying well-known ZIF-67 as a template [71]. As shown in Figure 6i, the thickness of the outer Ni-Co selenide shell is about $60 \mathrm{~nm}$, while the inner $\mathrm{CoSe}_{2}$ shell shows a very uneven thickness. 

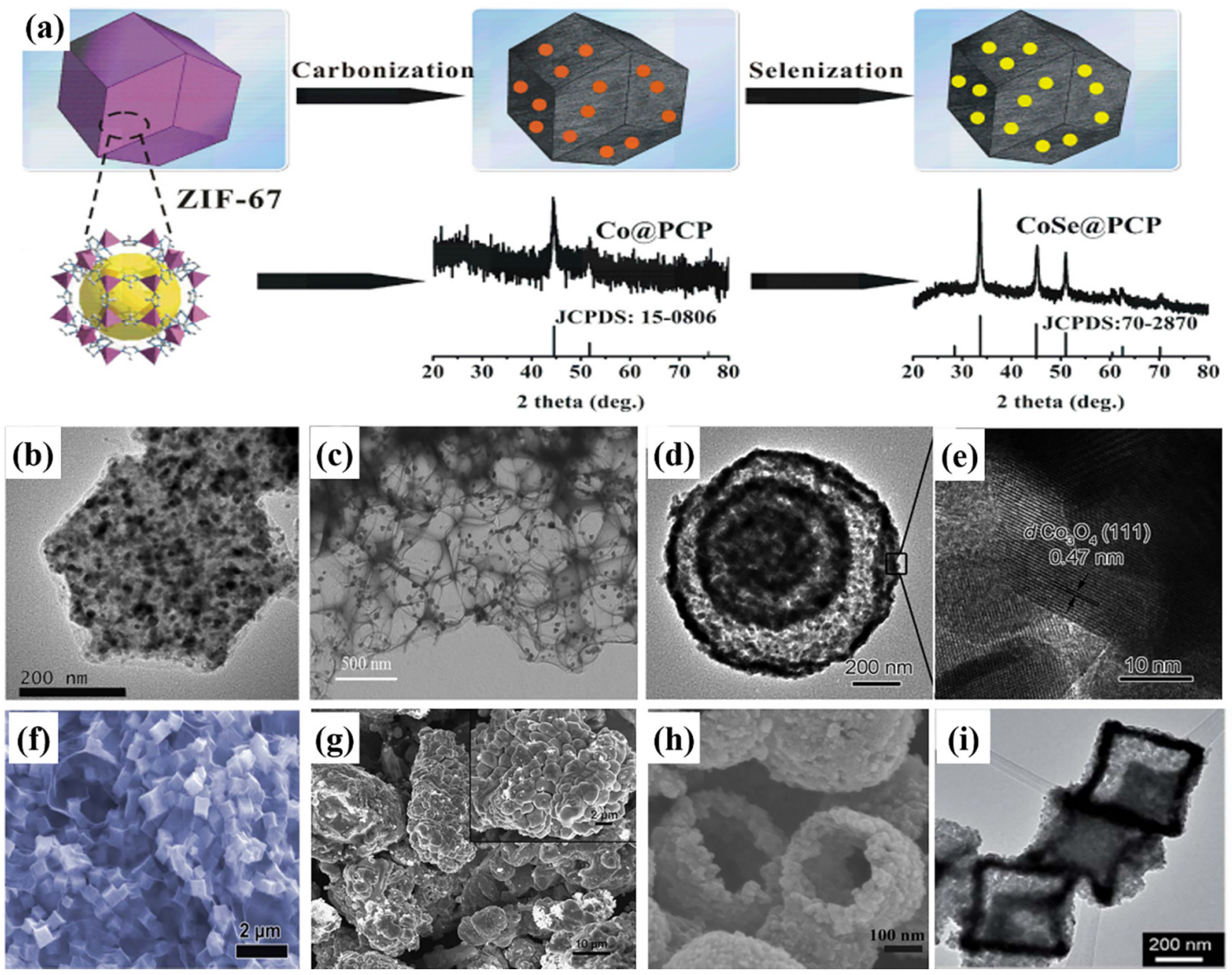

Figure 6. Preparation methods and morphology characterizations of cobalt-based composites. (a) Schematic illustration of the preparation of CoSe@porous carbon polyhedral (PCP). (b) TEM image of CoSe@PCP [68]. (c) TEM image of 3D spongy $\mathrm{CoS}_{2} / \mathrm{C}$ [12]. (d) TEM and (e) HRTEM images of triple-shelled $\mathrm{Co}_{3} \mathrm{O}_{4}$ hollow microspheres [69]. (f) SEM image of the reduced graphene oxide nanosheet (RGO@CoP@FeP) composite [70]. (g) SEM images of the $\mathrm{CoSn}_{2} / \mathrm{Sn}$ alloy [22]. (h) SEM image of the $\mathrm{Co}_{9} \mathrm{~S}_{8} / \mathrm{N}-\mathrm{C}$ hollow spheres [28]. (i) TEM image of the $\mathrm{Co} /(\mathrm{NiCo}) \mathrm{Se}_{2}$ nanocubes with a box-in-box structure [71].

\subsection{High-Energy Mechanical Milling}

As a facile preparation technology, high-energy mechanical milling (denoted as HEMM) has also been commonly applied to the fabrication of cobalt-based materials owing to its large-scale production $[34,42,45,80-83]$. For HEMM, the products are synthesized under high temperatures and pressures generated by highly energetic collisions of tiny balls.

Carbon-coated $\mathrm{CoP}_{3}\left(\mathrm{CoP}_{3} @ \mathrm{C}\right)$ nanocomposites were synthesized by HEMM methods with a certain proportion of phosphorus, cobalt powders and carbon black [80]. The synthesis of the schematic illustration is displayed in Figure 7a. HRTEM images shown in Figure $7 \mathrm{~b}$ reveal the presence of $\mathrm{CoP}_{3}$ nanoparticles and a carbon matrix. B. Scrosati's group successfully transformed a certain proportion of Sn, Co and graphite into a nanosized SnCoC-2 alloy through HEMM [34]. Figure 7c shows the SEM image of the obtained SnCoC-2 alloy. Li et al. prepared $\mathrm{Sn}_{30} \mathrm{Co}_{30} \mathrm{C}_{40}$ electrode materials by mechanical attrition with $\mathrm{CoSn}_{2}$, Co and graphite as the precursors [81]. SEM image of the obtained $\mathrm{Sn}_{30} \mathrm{Co}_{30} \mathrm{C}_{40}$ (Figure $7 \mathrm{~d}$ ) reveals that the average size of $\mathrm{Sn}_{30} \mathrm{Co}_{30} \mathrm{C}_{40}$ particles is about $1 \mu \mathrm{m}$. 

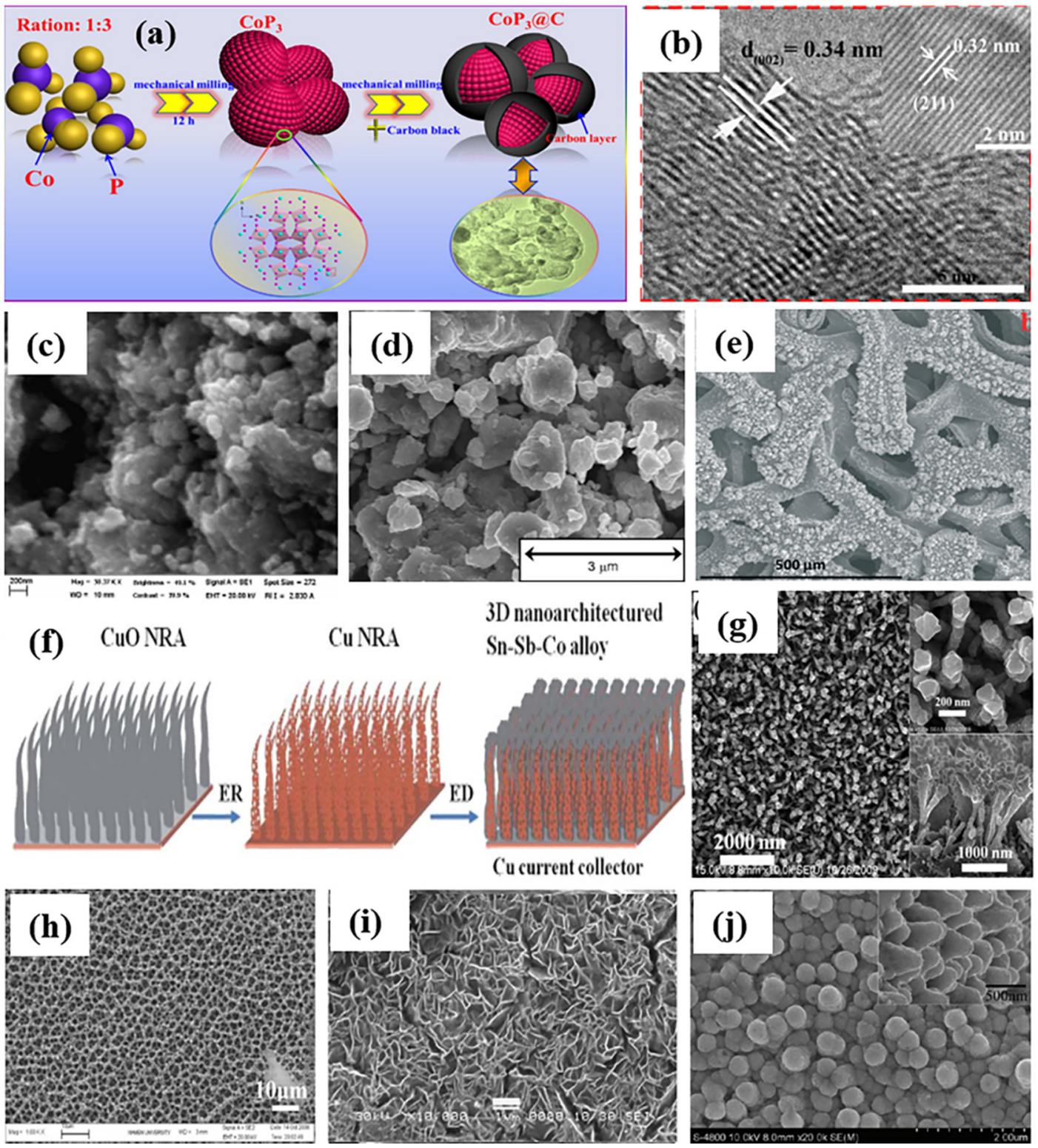

Figure 7. Preparation methods and morphology characterizations of cobalt-based composites. (a) Schematic illustration of the preparation of $\mathrm{CoP}_{3} @ \mathrm{C}$ microspheres. (b) HRTEM image of the $\mathrm{CoP}_{3} @ \mathrm{C}$ composite [80]. (c) SEM image of the SnCoC-2 sample [34]. (d) SEM image of the $\mathrm{Sn}_{30} \mathrm{Co}_{30} \mathrm{C}_{40}$ material [81]. (e) SEM image of the Co-Sn alloy after electrodeposition [84]. (f) Schematic illustration of the preparation of the Sn-Sb-Co alloy. (g) SEM images of the SnSbCo-3 electrode; the insets show a corresponding high-magnification image (top-right) and cross-sectional photos of the electrode (bottom-right) [85]. (h) SEM image of the porous SnCo alloy [86]. (i) SEM image of $\mathrm{Co}_{3} \mathrm{O}_{4}$ thin films synthesized by electrochemical deposition [87]. (j) SEM image of the Sn-Co alloy deposited on a Ni nanocon-array [38].

\subsection{Electrodeposition}

Electrodeposition, as a method for separating metals or alloys from their compounds, has been used in the preparation of cobalt-based electrode materials [18,38,43,84-89]. Ricardo's group prepared the Co-Sn alloy by depositing Sn and Co atoms through the co-electroplating method, according to moderate time and current density [84]. Figure 7e indicates that the deposited Co-Sn alloy covered the nickel substrate with certain roughness and protuberances. Sun's group reported a novel nanoarchitectured Sn-Sb-Co alloy through direct electrodeposition on a Cu nanoribbon array [85]. 
A schematic diagram of the preparation of 3D nanoarchitectured Sn-Sb-Co alloy is shown in Figure 7f. SEM images (Figure $7 \mathrm{~g}$ ) clearly reveal the 3D array nanostructure and thickness $(1500 \mathrm{~nm})$ of the Sn-Sb-Co alloy.

A porous Sn-Co alloy was fabricated by Sun's group via electrodepositing the Sn-Co ally on the porous $\mathrm{Cu}$ film prepared by electroless plating [86]. SEM image (Figure $7 \mathrm{~h}$ ) indicates that the Sn-Co alloy was uniformly covered on the porous $\mathrm{Cu}$ film. Porous $\mathrm{Co}_{3} \mathrm{O}_{4}$ nanostructured thin films were synthesized via a facile electrodepositing method (Figure 7i) [87]. SEM image reveals that the sample was composed of small nanoflakes of about $30-40 \mathrm{~nm}$ in thickness. Du et al. successfully a synthesized nanoarchitectured Sn-Co alloy electrode through a two-step electrodeposition [38]. The SEM image in Figure 7j evidently indicates that there is sufficient room between the Sn-Co cylinders

\subsection{Other Methods}

With the exception of these methods, many other practical approaches, such as electrospinning, magnetron sputtering, chemical vapor deposition (CVD) and electric arc melting, have been applied to synthesize cobalt-based electrode materials with novel nanostructures [19,44,50,90-96].

Electrospinning is a method to synthesize 1D nanomaterials by the breakdown of a high-polymer solution by a high-voltage electrostatic field $[24,93,97]$. Li's group distributed $\mathrm{CoSe}_{2}$ nanoparticles uniformly into nitrogen-doped carbon nanofibers through two steps, including the electrospinning approach and subsequent selenization process (Figure 8a) [24]. TEM image, HRTEM image and corresponding elements mapping clearly reveal that $\mathrm{CoSe}_{2}$ particles were well-wrapped in the N-doped nanofibers without aggregation, as shown in Figure 8b-d, respectively. Co-Sn/CNF composites were fabricated by Lee's group through electrospinning, followed with heat treatments [90]. SEM image of the $\mathrm{Co}-\mathrm{Sn} / \mathrm{CNF}$ surface shows that the average diameter of the fibers was $180 \mathrm{~nm}$ (Figure 8e). Wang et al. prepared carbon-encapsulated wire-in-tube $\mathrm{Co}_{3} \mathrm{O}_{4} / \mathrm{MnO}_{2}$ heterostructure nanofibers through electrospinning, followed by calcination [91]. The TEM image in Figure $8 \mathrm{f}$ shows a tubular structure with three layers, including an $\mathrm{MnO}_{2}$ wire, $\mathrm{Co}_{3} \mathrm{O}_{4}$ tube and carbon layer, subsequently. X-ray EDS in Figure $8 \mathrm{~g}$ clearly indicates the presence of $\mathrm{Mn}, \mathrm{Co}, \mathrm{O}, \mathrm{N}$ and $\mathrm{C}$ elements. Kim's group prepared 1D carbon nanofibers embedded with uniformly SnCo nanoparticles via electrospinning, followed by calcination [92]. SEM image in Figure $8 \mathrm{~h}$ shows that SnCo alloys with size distributions of 5-10 nm were homogeneously encapsulated in carbon nanofibers. Niu's group successfully synthesized a novel urchin-like sample composed of $\mathrm{CoSe}_{2}$ nanofibers rooted into the carbon nanofibers (CoSe @ $@ \mathrm{CNFs}$ ) [93]. In the preparation process, Niu's group firstly synthesized the precursor (Co@CNF) via electrospinning, followed by calcination, and subsequently prepared $\mathrm{CoSe}_{2} @ \mathrm{CNFs}$ through a hydrothermal selenation. TEM image and corresponding HRTEM image (inset) in Figure 8i obviously show that urchin-like $\mathrm{CoSe}_{2}$ nanorods with lengths of 50-100 nm and diameters of 20-30 nm are rooted into the electrospun CNFs. Qiu et al. employed the electrospinning method to synthesize $\mathrm{Co} / \mathrm{Co}_{3} \mathrm{O}_{4}$-carbon nanofibers with porous structures [94]. The $\mathrm{Co} / \mathrm{Co}_{3} \mathrm{O}_{4}$ nanoparticles were distributed throughout the carbon nanofiber, as shown in Figure 8j. 

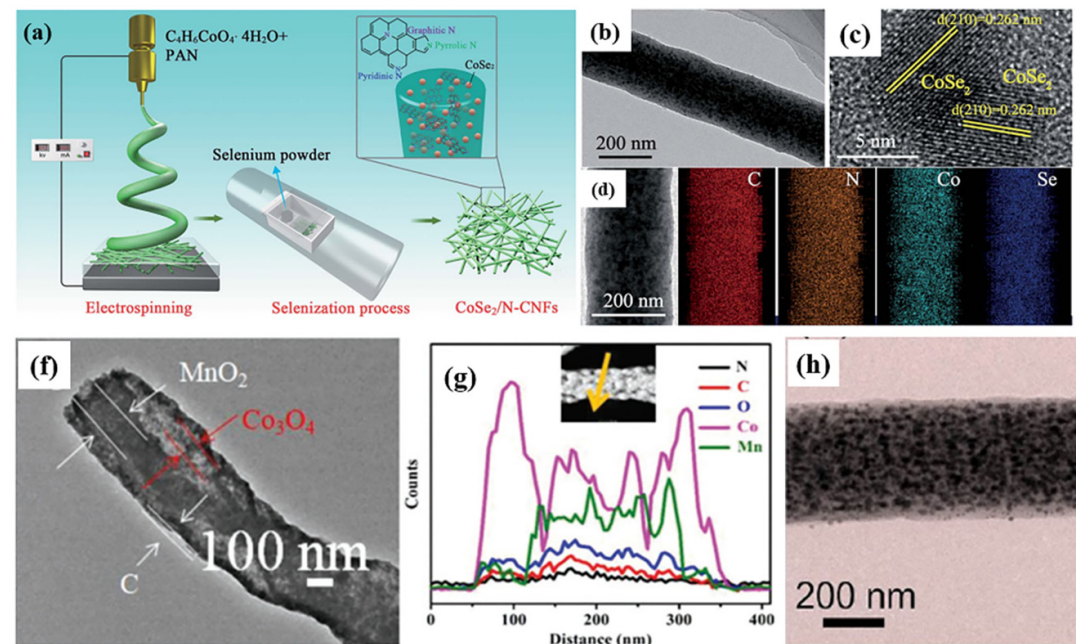

(h)
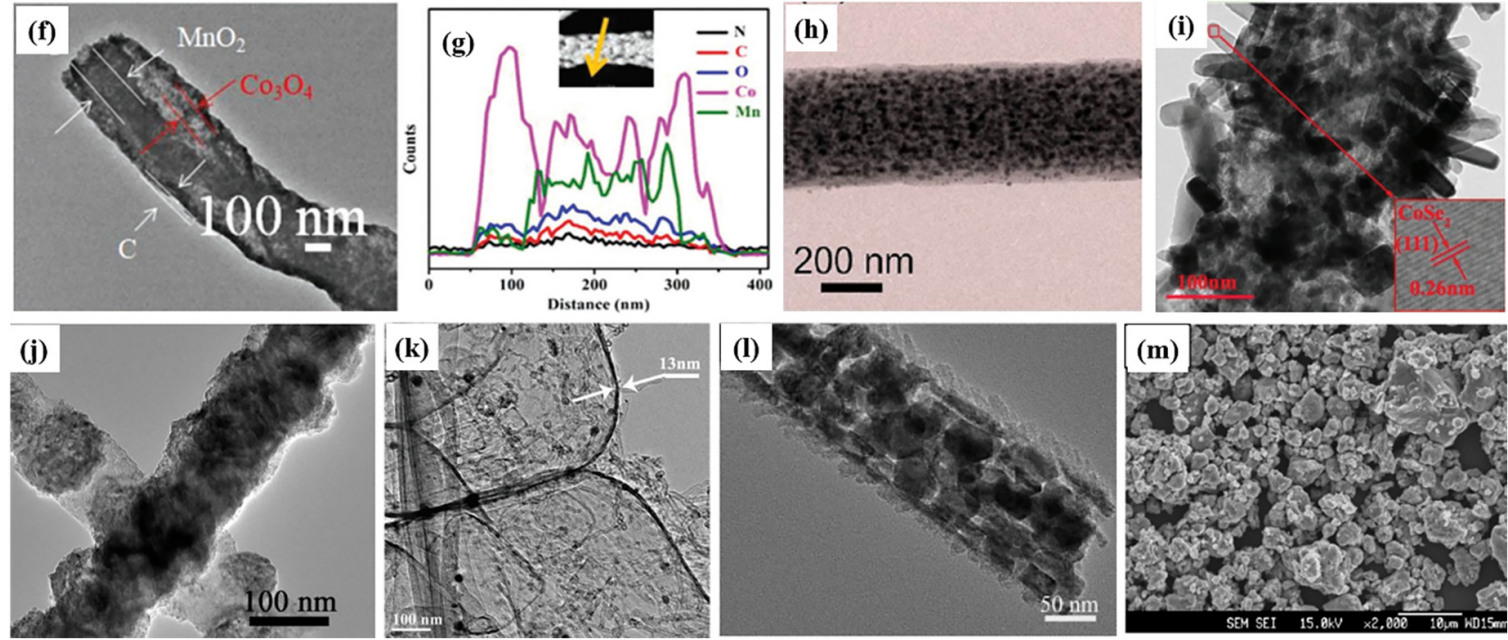

Figure 8. Preparation methods and morphology characterizations of cobalt-based composites. (a) Schematic illustration of the preparation of $\mathrm{CoSe}_{2} / \mathrm{N}$-carbon nanofibers (CNFs). (b) TEM image, (c) HRTEM image and (d) elemental mapping images of $\mathrm{CoSe}_{2} / \mathrm{N}-\mathrm{CNFs}$ [24]. (e) SEM image of Co-Sn/CNFs at $800{ }^{\circ} \mathrm{C}$ [90]. (f) TEM image and (g) HAADF-STEM-EDS line-scanning elemental mapping of $\mathrm{Co}_{3} \mathrm{O}_{4} / \mathrm{MnO}_{2} @ \mathrm{C}$ [91]. (h) TEM image of SnCo/PAN-CNFs [92]. (i) HRTEM image is taken from the selected zone [93]. (j) TEM image of $\mathrm{Co} / \mathrm{Co}_{3} \mathrm{O}_{4}-\mathrm{CNF}$-700 [94]. (k) TEM image of the SnCo@CNT-3DC composite [44]. (l) SEM image of $\mathrm{Co}_{3} \mathrm{O}_{4}$ nanowire arrays [95]. (m) SEM image of the $\mathrm{Co}_{2} \mathrm{MnSi}$ powder [96].

Chemical vapor deposition (CVD) processes have also been applied to synthesize cobalt-based electrode materials with multifarious structures [44,98-100]. He's group successfully prepared a novel structure via the CVD process [44]. The TEM image in Figure 8k clearly indicates a typical 0D-1D-3D hierarchical structure.

The magnetron sputtering method is also developed to prepare cobalt-based materials on substrates $[19,101]$. Chen et al. synthesized a $\mathrm{Co}_{3} \mathrm{O}_{4}-\mathrm{C}$ core-shell nanowire array via hydrothermal and magnetron sputtering. SEM image in Figure 81 shows that the $\mathrm{Co}_{3} \mathrm{O}_{4}$ nanowires were well-coated by carbon layers with a thickness of $18 \mathrm{~nm}$.

Furthermore, Yoon's group reported an electric arc-melting method to produce a $\mathrm{Co}_{2} \mathrm{MnSi}$ ingot [96]. Figure $8 \mathrm{~m}$ indicates that the synthesized materials exhibited a certain degree of aggregation.

Various strategies for the preparation of cobalt-based electrode materials are summarized in Table 2. Obviously, hydrothermal/solvothermal, heat treatment, galvanic replacement and HEMM can be used to prepare different types of cobalt-based materials due to their economy and simplicity naturally. Other methods like electrospinning, electrodeposition and magnetron sputtering are more likely to synthesize cobalt-based materials with specific morphologies and structures, such as nanofibers and films. The diversity of the preparation process ensures the variety of synthesized cobalt-based electrode materials, thereby increasing the opportunities of cobalt-based materials for real batteries. 
Table 2. Summary of the synthetic methods for cobalt-based electrode materials.

\begin{tabular}{|c|c|c|c|}
\hline Types of Materials & Synthetic Method & Co Source & Reference \\
\hline $\mathrm{Co}_{3} \mathrm{Sn}_{2} @ \mathrm{Co}-\mathrm{NG}$ & Hydrothermal $\left(250^{\circ} \mathrm{C}, 24 \mathrm{~h}\right)$ & $\mathrm{CoCl}_{2} \bullet 6 \mathrm{H}_{2} \mathrm{O}$ & [53] \\
\hline Special $\mathrm{CoSn}_{2} / \mathrm{Sn}$ alloy & Heat treatment $\left(800^{\circ} \mathrm{C}, \mathrm{Ar}\right)$ & $\mathrm{Co}_{3} \mathrm{O}_{4}$ & [22] \\
\hline Sn-Co-C ternary alloy & HEMM & Co powder & [34] \\
\hline $\mathrm{Sn}_{36} \mathrm{Co}_{41} \mathrm{C}_{23}$ alloy & Magnetron sputtering & Co powder & [19] \\
\hline $\mathrm{Sn}_{40} \mathrm{Co}_{40} \mathrm{C}_{20}$ & HEMM & Co powder & [45] \\
\hline Sn-Co-C composite & HEMM & $\mathrm{Co}\left(\mathrm{C}_{5} \mathrm{H}_{7} \mathrm{O}_{2}\right)_{3}$ & [42] \\
\hline Sn-Co alloy & Electrodepositing & $\mathrm{CoCl}_{2} \bullet 6 \mathrm{H}_{2} \mathrm{O}$ & [43] \\
\hline Sn-Co/CNTs & Galvanic replacement & $\mathrm{CoCl}_{2} \bullet 6 \mathrm{H}_{2} \mathrm{O}$ & [102] \\
\hline Sn-Co-C composite & HEMM & $\mathrm{CoO}$ & [83] \\
\hline Nano-sized Co-Sn alloy & Hydrothermal $\left(160^{\circ} \mathrm{C}, 48 \mathrm{~h}\right)$ & $\mathrm{CoCl}_{2} \bullet 6 \mathrm{H}_{2} \mathrm{O}$ & [55] \\
\hline $\mathrm{Sn}_{30} \mathrm{Co}_{30} \mathrm{C}_{40}$ alloy & Ball-milling & Co powder & [81] \\
\hline $\mathrm{CoSn}_{3}$ & Hydrothermal $\left(195^{\circ} \mathrm{C}, 1.5 \mathrm{~h}\right)$ & $\mathrm{CoCl}_{2} \bullet 6 \mathrm{H}_{2} \mathrm{O}$ & [56] \\
\hline Sn-Co alloy & Electrodepositing & $\mathrm{CoCl}_{2} \bullet 6 \mathrm{H}_{2} \mathrm{O}$ & [38] \\
\hline $\mathrm{CoSn}_{2}$ & Electrodepositing & Co electrode & [89] \\
\hline Nanostructure $\mathrm{Sn}-\mathrm{Co}-\mathrm{C}$ composites & Galvanic replacement & $\mathrm{Co}\left(\mathrm{CH}_{3} \mathrm{COO}\right)_{2} \bullet 4 \mathrm{H}_{2} \mathrm{O}$ & [103] \\
\hline porous Sn-Co alloy & Electrodepositing & $\mathrm{CoCl}_{2} \bullet 6 \mathrm{H}_{2} \mathrm{O}$ & [86] \\
\hline $\mathrm{CoSn}_{3}$-MWCNTs & Galvanic replacement & $\mathrm{CoCl}_{2} \bullet 6 \mathrm{H}_{2} \mathrm{O}$ & [104] \\
\hline $\mathrm{Sn}_{54} \mathrm{Sb}_{41} \mathrm{Co}_{5}$ alloy & Electrodepositing & $\mathrm{CoCl}_{2} \bullet 6 \mathrm{H}_{2} \mathrm{O}$ & [85] \\
\hline $\mathrm{CoSn}_{5}$ nanospheres & Galvanic replacement & $\mathrm{CoCl}_{2} \bullet 6 \mathrm{H}_{2} \mathrm{O}$ & [105] \\
\hline $\begin{array}{c}\text { Grapheme wrapped-SnCo } \\
\text { nanoparticles }\end{array}$ & Galvanic replacement & $\mathrm{CoCl}_{2} \bullet 6 \mathrm{H}_{2} \mathrm{O}$ & [106] \\
\hline $\mathrm{CoSn}_{2}$ alloy & Electrodepositing & $\mathrm{CoCl}_{2} \bullet 6 \mathrm{H}_{2} \mathrm{O}$ & [84] \\
\hline Sn-Co-CNT@CNT & Galvanic replacement & $\mathrm{CoCl}_{2} \bullet 6 \mathrm{H}_{2} \mathrm{O}$ & [67] \\
\hline Co-Sn/C nanofiber & Electrodepositing & $\mathrm{C}_{4} \mathrm{H}_{6} \mathrm{CoO}_{4}$ & [90] \\
\hline $\mathrm{SnCo} / \mathrm{C}$ nanofibers & Electrospinning & $\mathrm{C}_{4} \mathrm{H}_{6} \mathrm{CoO}_{4}$ & [92] \\
\hline Sn-Co@PMMA nanospheres & Galvanic replacement & $\mathrm{C}_{4} \mathrm{H}_{6} \mathrm{CoO}_{4}$ & [40] \\
\hline Amorphous $\mathrm{Co}_{3} \mathrm{Sn}_{2}$ & Hydrothermal $\left(180^{\circ} \mathrm{C}, 24 \mathrm{~h}\right)$ & $\mathrm{CoCl}_{2} \bullet 6 \mathrm{H}_{2} \mathrm{O}$ & [107] \\
\hline SnCo@CNT-3DC & CVD & $\mathrm{CoCl}_{2} \bullet 6 \mathrm{H}_{2} \mathrm{O}$ & [44] \\
\hline Sn-Co@C & Galvanic replacement & $\mathrm{Co}\left(\mathrm{NO}_{3}\right)_{2} \bullet 6 \mathrm{H}_{2} \mathrm{O}$ & [64] \\
\hline carbon encapsulated Sn-Co alloy & Heat treatment $\left(800^{\circ} \mathrm{C}, \mathrm{Ar} / \mathrm{H}_{2}\right)$ & $\mathrm{Co}_{3} \mathrm{O}_{4}$ & [41] \\
\hline $\mathrm{NiCo}_{2} \mathrm{O}_{4}$ powders & Heat treatment $\left(320^{\circ} \mathrm{C}\right.$, air $)$ & $\mathrm{CoC}_{2} \mathrm{O}_{4}$ & [72] \\
\hline $\mathrm{Co}_{2} \mathrm{MnSi}$ & Arc-melting & Co powder & [96] \\
\hline $\mathrm{Sb}-\mathrm{Co}-\mathrm{P}$ & Electroplating & $\mathrm{CoCl}_{2} \bullet 6 \mathrm{H}_{2} \mathrm{O}$ & [50] \\
\hline CoSnC alloy & Galvanic replacement & $\mathrm{CoSO}_{4} \bullet 6 \mathrm{H}_{2} \mathrm{O}$ & [108] \\
\hline $\mathrm{Co}_{2} \mathrm{SnO}_{4} \mathrm{HC} @ \mathrm{rGO}$ & Heat treatment $\left(900^{\circ} \mathrm{C}, \mathrm{Ar}\right)$ & $\mathrm{CoCl}_{2} \bullet 6 \mathrm{H}_{2} \mathrm{O}$ & [39] \\
\hline $\mathrm{CoSnO}_{3} / \mathrm{GN} / \mathrm{CNTs}$ & Galvanic replacement & $\mathrm{CoCl}_{2} \bullet 6 \mathrm{H}_{2} \mathrm{O}$ & [109] \\
\hline $\mathrm{CoMn}_{2} \mathrm{O}_{4}$ & Hydrothermal $\left(180^{\circ} \mathrm{C}, 10 \mathrm{~h}\right)$ & $\begin{array}{c}\mathrm{Co}\left(\mathrm{CH}_{3} \mathrm{COO}\right)_{2} \\
\bullet 4 \mathrm{H}_{2} \mathrm{O}\end{array}$ & [51] \\
\hline $\mathrm{CoSnO}_{3}$ & Galvanic replacement & $\mathrm{CoCl}_{2} \bullet 6 \mathrm{H}_{2} \mathrm{O}$ & [52] \\
\hline $\mathrm{Co}_{3} \mathrm{O}_{4}$ & Heat treatment $\left(800^{\circ} \mathrm{C}\right.$, Air) & $\mathrm{CoCO}_{3}$ & [15] \\
\hline
\end{tabular}


Table 2. Cont.

\begin{tabular}{|c|c|c|c|}
\hline Types of Materials & Synthetic Method & Co Source & Reference \\
\hline $\mathrm{Co}_{3} \mathrm{O}_{4}$ nanotubes & Heat treatment $\left(500{ }^{\circ} \mathrm{C}\right.$, Oxygen $)$ & $\mathrm{Co}_{4}(\mathrm{CO})_{12}$ & [73] \\
\hline $\mathrm{Co}_{3} \mathrm{O}_{4}$ thin films & Electrodepositing & $\mathrm{Co}\left(\mathrm{NO}_{3}\right)_{2}$ & [88] \\
\hline porous $\mathrm{Co}_{3} \mathrm{O}_{4}$ thin films & Electrodepositing & $\mathrm{Co}\left(\mathrm{NO}_{3}\right)_{2}$ & [87] \\
\hline $\mathrm{Co}_{3} \mathrm{O}_{4}$ microspheres & Hydrothermal $\left(200^{\circ} \mathrm{C}, 3 \mathrm{~h}\right)$ & $\mathrm{Co}\left(\mathrm{NO}_{3}\right)_{2} \bullet 6 \mathrm{H}_{2} \mathrm{O}$ & {$[21]$} \\
\hline needlelike $\mathrm{Co}_{3} \mathrm{O}_{4}$ nanotubes & Galvanic replacement & $\mathrm{Co}\left(\mathrm{NO}_{3}\right)_{2} \bullet 6 \mathrm{H}_{2} \mathrm{O}$ & {$[65]$} \\
\hline $\mathrm{Co}_{3} \mathrm{O}_{4}$ nanosheets & Hydrothermal $\left(140^{\circ} \mathrm{C}, 20 \mathrm{~h}\right)$ & $\mathrm{CoCl}_{2} \bullet 6 \mathrm{H}_{2} \mathrm{O}$ & {$[54]$} \\
\hline agglomerated $\mathrm{Co}_{3} \mathrm{O}_{4}$ & Heat treatment $\left(600{ }^{\circ} \mathrm{C}\right.$, Air $)$ & $\mathrm{Co}_{3}(\mathrm{NDC})_{3}(\mathrm{DMF})_{4}$ & {$[74]$} \\
\hline macroporous $\mathrm{Co}_{3} \mathrm{O}_{4}$ platelets & Heat treatment $\left(450{ }^{\circ} \mathrm{C}\right.$, Air $)$ & $\mathrm{CoCl}_{2} \bullet 6 \mathrm{H}_{2} \mathrm{O}$ & [75] \\
\hline $\mathrm{Co}_{3} \mathrm{O}_{4} /$ graphene & Galvanic replacement & $\begin{array}{c}\left(\mathrm{C}_{2} \mathrm{H}_{3} \mathrm{O}_{2}\right)_{2} \mathrm{Co} \\
\bullet 4 \mathrm{H}_{2} \mathrm{O}\end{array}$ & [110] \\
\hline $\mathrm{Co}_{3} \mathrm{O}_{4} /$ graphene & Galvanic replacement & $\mathrm{CoCl}_{2} \bullet 6 \mathrm{H}_{2} \mathrm{O}$ & [111] \\
\hline $\mathrm{Co}_{3} \mathrm{O}_{4}$ porous nanocages & Heat treatment $\left(400^{\circ} \mathrm{C}\right.$, Air $)$ & $\begin{array}{c}\left(\mathrm{C}_{2} \mathrm{H}_{3} \mathrm{O}_{2}\right)_{2} \mathrm{Co} \\
\bullet 4 \mathrm{H}_{2} \mathrm{O}\end{array}$ & [76] \\
\hline single-crystalline $\mathrm{Co}_{3} \mathrm{O}_{4}$ nanobelts & Hydrothermal $\left(120^{\circ} \mathrm{C}, 24 \mathrm{~h}\right)$ & $\mathrm{Co}\left(\mathrm{NO}_{3}\right)_{2} \bullet 6 \mathrm{H}_{2} \mathrm{O}$ & [57] \\
\hline $\mathrm{C} @ \mathrm{Co}_{3} \mathrm{O}_{4}$ & Hydrothermal $\left(180^{\circ} \mathrm{C}, 8 \mathrm{~h}\right)$ & $\mathrm{Co}\left(\mathrm{NO}_{3}\right)_{2} \bullet 6 \mathrm{H}_{2} \mathrm{O}$ & [112] \\
\hline $\begin{array}{c}\mathrm{Co}_{3} \mathrm{O}_{4} \text { nanorods/graphene } \\
\text { nanosheets }\end{array}$ & Hydrothermal $\left(120{ }^{\circ} \mathrm{C}, 12 \mathrm{~h}\right)$ & $\mathrm{CoSO}_{4} \bullet 7 \mathrm{H}_{2} \mathrm{O}$ & [113] \\
\hline mesoporous $\mathrm{Co}_{3} \mathrm{O}_{4}$ & Galvanic replacement & $\mathrm{Co}\left(\mathrm{NO}_{3}\right)_{2} \bullet 6 \mathrm{H}_{2} \mathrm{O}$ & [114] \\
\hline $\mathrm{Co}_{3} \mathrm{O}_{4}$ nanocages & Heat treatment $\left(550{ }^{\circ} \mathrm{C}\right.$, Air $)$ & $\left(\mathrm{C}_{2} \mathrm{H}_{3} \mathrm{O}_{2}\right)_{2} \mathrm{Co} \bullet \mathrm{nH}_{2} \mathrm{O}$ & [77] \\
\hline graphene $/ \mathrm{Co}_{3} \mathrm{O}_{4}$ & Hydrothermal $\left(80^{\circ} \mathrm{C}, 4 \mathrm{~h}\right)$ & $\mathrm{CoCl}_{2} \bullet 6 \mathrm{H}_{2} \mathrm{O}$ & [115] \\
\hline CoO/graphene & Heat treatment $\left(350{ }^{\circ} \mathrm{C}, \mathrm{Ar} / \mathrm{H}_{2}\right)$ & $\mathrm{Co}\left(\mathrm{NO}_{3}\right)_{2} \bullet 6 \mathrm{H}_{2} \mathrm{O}$ & [78] \\
\hline $\mathrm{Co}_{3} \mathrm{O}_{4} /$ graphene & Galvanic replacement & $\left(\mathrm{C}_{2} \mathrm{H}_{3} \mathrm{O}_{2}\right)_{2} \mathrm{Co} \bullet 4 \mathrm{H}_{2} \mathrm{O}$ & [116] \\
\hline $\begin{array}{l}\text { multi-shelled } \mathrm{Co}_{3} \mathrm{O}_{4} \text { hollow } \\
\text { microspheres }\end{array}$ & Heat treatment $\left(500^{\circ} \mathrm{C}\right.$, Air) & $\mathrm{Co}(\mathrm{Ac})_{2} \bullet 4 \mathrm{H}_{2} \mathrm{O}$ & [69] \\
\hline shale-like $\mathrm{Co}_{3} \mathrm{O}_{4}$ & Heat treatment $\left(400{ }^{\circ} \mathrm{C}\right.$, Air $)$ & $\left(\mathrm{C}_{2} \mathrm{H}_{3} \mathrm{O}_{2}\right)_{2} \mathrm{Co} \bullet 4 \mathrm{H}_{2} \mathrm{O}$ & [35] \\
\hline hierarchical $\mathrm{Co}_{3} \mathrm{O}_{4} / \mathrm{CNTs}$ & Galvanic replacement & $\mathrm{Co}\left(\mathrm{NO}_{3}\right)_{2} \bullet 6 \mathrm{H}_{2} \mathrm{O}$ & [63] \\
\hline $\begin{array}{l}\text { bowl-like hollow } \mathrm{Co}_{3} \mathrm{O}_{4} \\
\text { microspheres }\end{array}$ & Heat treatment $\left(400^{\circ} \mathrm{C}\right.$, Air $)$ & $\left(\mathrm{C}_{2} \mathrm{H}_{3} \mathrm{O}_{2}\right)_{2} \mathrm{Co} \bullet 4 \mathrm{H}_{2} \mathrm{O}$ & [117] \\
\hline mesoporous $\mathrm{Co}_{3} \mathrm{O}_{4}$ nanoflakes & Heat treatment $\left(250^{\circ} \mathrm{C}\right.$, Air $)$ & $\mathrm{CoCO}_{3}$ & [118] \\
\hline $\begin{array}{c}\text { hollow structured } \mathrm{Co}_{3} \mathrm{O}_{4} \\
\text { nanoparticles }\end{array}$ & Heat treatment $\left(400^{\circ} \mathrm{C}\right.$, Air $)$ & $\mathrm{CoCl}_{2} \bullet 6 \mathrm{H}_{2} \mathrm{O}$ & [119] \\
\hline $\mathrm{Co}_{3} \mathrm{O}_{4} / \mathrm{MCNTs}$ & Heat treatment $\left(500{ }^{\circ} \mathrm{C}\right.$, Air) & $\mathrm{CoCO}_{3}$ & [120] \\
\hline $\begin{array}{c}\text { peapod-like } \mathrm{Co}_{3} \mathrm{O}_{4} @ \text { carbon } \\
\text { nanotube }\end{array}$ & Heat treatment $\left(450^{\circ} \mathrm{C}, \mathrm{Ar}\right)$ & $\mathrm{Co}\left(\mathrm{NO}_{3}\right)_{2} \bullet 6 \mathrm{H}_{2} \mathrm{O}$ & [121] \\
\hline $\mathrm{Co}_{3} \mathrm{O}_{4} / \mathrm{CNT}$ nanocomposites & Heat treatment $\left(300^{\circ} \mathrm{C}, \mathrm{Air}\right)$ & $\mathrm{CoCl}_{2} \bullet 6 \mathrm{H}_{2} \mathrm{O}$ & {$[122]$} \\
\hline layer-by-layer $\mathrm{Co}_{3} \mathrm{O}_{4}$ /graphene & Hydrothermal $\left(170^{\circ} \mathrm{C}, 15 \mathrm{~h}\right)$ & $\left(\mathrm{C}_{2} \mathrm{H}_{3} \mathrm{O}_{2}\right)_{2} \mathrm{Co} \bullet 4 \mathrm{H}_{2} \mathrm{O}$ & {$[123]$} \\
\hline $\mathrm{Co}_{3} \mathrm{O}_{4} / \mathrm{CNTs}$ nanotubes & Hydrothermal $\left(120^{\circ} \mathrm{C}, 2 \mathrm{~h}\right)$ & $\left(\mathrm{C}_{2} \mathrm{H}_{3} \mathrm{O}_{2}\right)_{2} \mathrm{Co} \bullet 4 \mathrm{H}_{2} \mathrm{O}$ & [62] \\
\hline $\mathrm{Co} / \mathrm{Co}_{3} \mathrm{O}_{4}$ nanoparticles & Electrospinning & $\mathrm{CoCl}_{2} \bullet 6 \mathrm{H}_{2} \mathrm{O}$ & [94] \\
\hline $\mathrm{Co}_{3} \mathrm{O}_{4} @ \mathrm{NC}$ & Heat treatment $\left(550^{\circ} \mathrm{C}, \mathrm{Ar}\right)$ & ZIF-67 & [124] \\
\hline $\begin{array}{c}\text { carbon doped } \mathrm{Co}_{3} \mathrm{O}_{4} \text { hollow } \\
\text { nanofibers }\end{array}$ & Hydrothermal $\left(180^{\circ} \mathrm{C}, 12 \mathrm{~h}\right)$ & $\mathrm{Co}\left(\mathrm{NO}_{3}\right)_{2} \bullet 6 \mathrm{H}_{2} \mathrm{O}$ & [125] \\
\hline Ni-doped $\mathrm{Co} / \mathrm{CoO} / \mathrm{NC}$ hybrid & Heat treatment $\left(500{ }^{\circ} \mathrm{C}, \mathrm{Ar}\right)$ & $\mathrm{Co}\left(\mathrm{NO}_{3}\right)_{2} \bullet 6 \mathrm{H}_{2} \mathrm{O}$ & [126] \\
\hline
\end{tabular}


Table 2. Cont.

\begin{tabular}{|c|c|c|c|}
\hline Types of Materials & Synthetic Method & Co Source & Reference \\
\hline hollow $\mathrm{Co}_{3} \mathrm{O}_{4} / \mathrm{NGC}$ & Heat treatment & $\mathrm{Co}\left(\mathrm{NO}_{3}\right)_{2} \bullet 6 \mathrm{H}_{2} \mathrm{O}$ & {$[127]$} \\
\hline $\begin{array}{c}\text { starfish-like } \mathrm{Co}_{3} \mathrm{O}_{4} @ \text { nitrogen-doped } \\
\text { carbon }\end{array}$ & Heat treatment & $\mathrm{Co}\left(\mathrm{NO}_{3}\right)_{2} \bullet 6 \mathrm{H}_{2} \mathrm{O}$ & [128] \\
\hline yolk-shell $\mathrm{Co}_{3} \mathrm{O}_{4} / \mathrm{C}$ dodecahedrons & Heat treatment $\left(350^{\circ} \mathrm{C}\right.$, Air $)$ & $\mathrm{Co}\left(\mathrm{NO}_{3}\right)_{2} \bullet 6 \mathrm{H}_{2} \mathrm{O}$ & [129] \\
\hline hexagonal $\mathrm{Co}_{3} \mathrm{O}_{4}$ nanosheets & Hydrothermal $\left(120^{\circ} \mathrm{C}, 10 \mathrm{~h}\right)$ & $\mathrm{Co}\left(\mathrm{NO}_{3}\right)_{2} \bullet 6 \mathrm{H}_{2} \mathrm{O}$ & [130] \\
\hline $\begin{array}{l}\text { ultrathin mesoporous } \mathrm{Co}_{3} \mathrm{O}_{4} \\
\text { nanosheet }\end{array}$ & Heat treatment $\left(450^{\circ} \mathrm{C}\right.$, Air $)$ & $\mathrm{Co}\left(\mathrm{NO}_{3}\right)_{2} \bullet 6 \mathrm{H}_{2} \mathrm{O}$ & [131] \\
\hline flower-like $\mathrm{Co}_{3} \mathrm{O}_{4} / \mathrm{C}$ nanosheets & Heat treatment $\left(500{ }^{\circ} \mathrm{C}\right.$, Air) & $\mathrm{Co}\left(\mathrm{NO}_{3}\right)_{2} \bullet 6 \mathrm{H}_{2} \mathrm{O}$ & [132] \\
\hline $\mathrm{Co}_{3} \mathrm{O}_{4} / \mathrm{GF}$ & Heat treatment $\left(300^{\circ} \mathrm{C}\right.$, Air $)$ & $\mathrm{Co}\left(\mathrm{NO}_{3}\right)_{2} \bullet 6 \mathrm{H}_{2} \mathrm{O}$ & [133] \\
\hline ES- $\mathrm{CNCO}_{3} \mathrm{O}_{4}$ fibers & Heat treatment $\left(800^{\circ} \mathrm{C}, \mathrm{Ar} / \mathrm{H}_{2}\right)$ & $\mathrm{Co}\left(\mathrm{NO}_{3}\right)_{2} \bullet 6 \mathrm{H}_{2} \mathrm{O}$ & {$[11]$} \\
\hline $\mathrm{Co}_{3} \mathrm{O}_{4} / \mathrm{MnO}_{2} @ \mathrm{C}$ & Electrospinning & $\left(\mathrm{C}_{2} \mathrm{H}_{3} \mathrm{O}_{2}\right)_{2} \mathrm{Co} \bullet 4 \mathrm{H}_{2} \mathrm{O}$ & {$[91]$} \\
\hline $\mathrm{CoP}_{3}$ & Electrodeposition & $\mathrm{CoO}$ & [18] \\
\hline $\mathrm{CoP}_{\mathrm{x}}$ & Ball milling & Co powder & [82] \\
\hline $\mathrm{Co}_{2} \mathrm{P}$ & Electrodeposition & $\mathrm{CoCl}_{2} \bullet 6 \mathrm{H}_{2} \mathrm{O}$ & [46] \\
\hline $\mathrm{Co}_{x} \mathrm{P}$ & Heat treatment $\left(320^{\circ} \mathrm{C}, \mathrm{Ar}\right)$ & $\left(\mathrm{C}_{2} \mathrm{H}_{3} \mathrm{O}_{2}\right)_{2} \mathrm{Co} \bullet 4 \mathrm{H}_{2} \mathrm{O}$ & {$[79]$} \\
\hline $\mathrm{Co}_{x} \mathrm{P}$ & HEMM & Co powder & [134] \\
\hline $\mathrm{CoP} / \mathrm{RGO}$ & Hydrothermal $\left(180^{\circ} \mathrm{C}, 16 \mathrm{~h}\right)$ & $\mathrm{CoCl}_{2} \bullet 6 \mathrm{H}_{2} \mathrm{O}$ & [135] \\
\hline CoP microflake & Heat treatment $\left(350^{\circ} \mathrm{C}, \mathrm{Ar}\right)$ & $\mathrm{Co}\left(\mathrm{NO}_{3}\right)_{2} \bullet 6 \mathrm{H}_{2} \mathrm{O}$ & {$[136]$} \\
\hline CoP nanorod & Hydrothermal $\left(100^{\circ} \mathrm{C}, 12 \mathrm{~h}\right)$ & $\mathrm{Co}\left(\mathrm{NO}_{3}\right)_{2} \bullet 6 \mathrm{H}_{2} \mathrm{O}$ & [61] \\
\hline honeycomb-like $\mathrm{CoP} / \mathrm{Co}_{2} \mathrm{P}$ & Heat treatment $\left(600^{\circ} \mathrm{C}, \mathrm{Ar}\right)$ & $\mathrm{CoCl}_{2} \bullet 6 \mathrm{H}_{2} \mathrm{O}$ & {$[137]$} \\
\hline $\begin{array}{l}\text { core-shell CoP/FeP porous } \\
\text { microcubes }\end{array}$ & Heat treatment $\left(300^{\circ} \mathrm{C}, \mathrm{Ar}\right)$ & $\left(\mathrm{C}_{2} \mathrm{H}_{3} \mathrm{O}_{2}\right)_{2} \mathrm{Co} \bullet 4 \mathrm{H}_{2} \mathrm{O}$ & [70] \\
\hline mesoporous CoP nanorods & Hydrothermal $\left(120^{\circ} \mathrm{C}, 6 \mathrm{~h}\right)$ & $\mathrm{Co}\left(\mathrm{NO}_{3}\right)_{2} \bullet 6 \mathrm{H}_{2} \mathrm{O}$ & {$[138]$} \\
\hline $\mathrm{Co}_{2} \mathrm{P}-\mathrm{Co} /$ graphene & Galvanic replacement & $\left(\mathrm{C}_{2} \mathrm{H}_{3} \mathrm{O}_{2}\right)_{2} \mathrm{Co} \bullet 4 \mathrm{H}_{2} \mathrm{O}$ & [139] \\
\hline $\mathrm{A}-\mathrm{Co}_{2} \mathrm{P} / \mathrm{C}_{\mathrm{x}} \mathrm{N}_{\mathrm{y}} \mathrm{B}_{\mathrm{z}}-650$ & Heat treatment $\left(650^{\circ} \mathrm{C}, \mathrm{Ar}\right)$ & $\mathrm{Co}\left(\mathrm{NO}_{3}\right)_{2} \bullet 6 \mathrm{H}_{2} \mathrm{O}$ & [140] \\
\hline $\mathrm{CoP} / \mathrm{CNS}$ & Hydrothermal $\left(120^{\circ} \mathrm{C}, 6 \mathrm{~h}\right)$ & $\mathrm{Co}\left(\mathrm{NO}_{3}\right)_{2} \bullet 6 \mathrm{H}_{2} \mathrm{O}$ & [13] \\
\hline CoP@NPPCS & Heat treatment $\left(900^{\circ} \mathrm{C}, \mathrm{Ar}\right)$ & $\left(\mathrm{C}_{2} \mathrm{H}_{3} \mathrm{O}_{2}\right)_{2} \mathrm{Co} \bullet 4 \mathrm{H}_{2} \mathrm{O}$ & [141] \\
\hline CoP hollow nanorods/graphene & Hydrothermal $\left(140^{\circ} \mathrm{C}, 10 \mathrm{~h}\right)$ & $\mathrm{CoCl}_{2} \bullet 6 \mathrm{H}_{2} \mathrm{O}$ & [25] \\
\hline $\begin{array}{c}\text { carbon-encapsulated CoP } \\
\text { nanoparticles }\end{array}$ & Heat treatment $\left(850^{\circ} \mathrm{C}, \mathrm{Ar}\right)$ & $\mathrm{Co}\left(\mathrm{NO}_{3}\right)_{2} \bullet 6 \mathrm{H}_{2} \mathrm{O}$ & [142] \\
\hline $\mathrm{Co}_{2} \mathrm{P} @ \mathrm{~N}-\mathrm{C} @ \mathrm{rGO}$ & Heat treatment $\left(900^{\circ} \mathrm{C}, \mathrm{Ar}\right)$ & $\mathrm{Co}\left(\mathrm{NO}_{3}\right)_{2} \bullet 6 \mathrm{H}_{2} \mathrm{O}$ & {$[143]$} \\
\hline $\mathrm{CoP}_{3} @$ Ppy microcubes & Coprecipitation\& Parkerizing & $\left(\mathrm{C}_{2} \mathrm{H}_{3} \mathrm{O}_{2}\right)_{2} \mathrm{Co} \bullet 4 \mathrm{H}_{2} \mathrm{O}$ & [144] \\
\hline carbon coated $\mathrm{CoP}_{3}$ & HEMM & Co powder & {$[80]$} \\
\hline $\mathrm{Co}_{9} \mathrm{~S}_{8} @ \mathrm{C}$ nanoparticles & Hydrothermal \& heat treatment & $\mathrm{Co}\left(\mathrm{NO}_{3}\right)_{2} \bullet 6 \mathrm{H}_{2} \mathrm{O}$ & [23] \\
\hline $\begin{array}{c}\text { 3D spongy } \mathrm{CoS}_{2} \\
\text { nanoparticles/carbon }\end{array}$ & $\begin{array}{l}\text { Freeze-dry \&heat treatment } \\
\text { \&hydrothermal }\end{array}$ & $\mathrm{Co}\left(\mathrm{NO}_{3}\right)_{2} \bullet 6 \mathrm{H}_{2} \mathrm{O}$ & [12] \\
\hline hollow $\mathrm{Co}_{9} \mathrm{~S}_{8} @ \mathrm{C}$ & Heat treatment \& sulfuration & $\left(\mathrm{C}_{2} \mathrm{H}_{3} \mathrm{O}_{2}\right)_{2} \mathrm{Co} \bullet 4 \mathrm{H}_{2} \mathrm{O}$ & {$[145]$} \\
\hline CoS@S-doped OLC & Hydrothermal \& heat treatment & $\mathrm{Co}\left(\mathrm{NO}_{3}\right)_{2} \bullet 6 \mathrm{H}_{2} \mathrm{O}$ & [146] \\
\hline $\mathrm{SnS}_{2} @ \mathrm{CoS}_{2}-\mathrm{rGO}$ & Hydrothermal $\left(180^{\circ} \mathrm{C}, 24 \mathrm{~h}\right)$ & $\mathrm{CoCl}_{2} \bullet 6 \mathrm{H}_{2} \mathrm{O}$ & {$[147]$} \\
\hline $\mathrm{CoS}_{2} @ \mathrm{NCH}$ & Solvothermal \& heat treatment & $\mathrm{Co}\left(\mathrm{NO}_{3}\right)_{2} \bullet 6 \mathrm{H}_{2} \mathrm{O}$ & [148] \\
\hline $\mathrm{CoS}_{2} / \mathrm{C}$ micropolyhedron & Heat treatment $\left(900^{\circ} \mathrm{C}, \mathrm{N}_{2}\right)$ & $\mathrm{Co}\left(\mathrm{NO}_{3}\right)_{2} \bullet 6 \mathrm{H}_{2} \mathrm{O}$ & [33] \\
\hline
\end{tabular}


Table 2. Cont.

\begin{tabular}{|c|c|c|c|}
\hline Types of Materials & Synthetic Method & Co Source & Reference \\
\hline CoS-24 & Solvothermal $\left(180^{\circ} \mathrm{C} 24 \mathrm{~h}\right)$ & $\mathrm{CoCl}_{2} \bullet 6 \mathrm{H}_{2} \mathrm{O}$ & [149] \\
\hline CNT@NC@CuCo $\mathrm{S}_{4}$ & Solvothermal $\left(200^{\circ} \mathrm{C} 12 \mathrm{~h}\right)$ & $\left(\mathrm{C}_{2} \mathrm{H}_{3} \mathrm{O}_{2}\right)_{2} \mathrm{Co} \bullet 4 \mathrm{H}_{2} \mathrm{O}$ & [59] \\
\hline $\mathrm{Cog}_{8} \mathrm{~S}_{8}-\mathrm{QDs} @ \mathrm{NC}$ & Heat treatment $\left(750^{\circ} \mathrm{C}, \mathrm{Ar}\right)$ & $\mathrm{CoCl}_{2} \bullet 6 \mathrm{H}_{2} \mathrm{O}$ & [30] \\
\hline $\mathrm{TiO}_{2}$ nanobelts@Cog $\mathrm{S}_{8}$ & Hydrothermal \& heat treatment & $\left(\mathrm{C}_{2} \mathrm{H}_{3} \mathrm{O}_{2}\right)_{2} \mathrm{Co} \bullet 4 \mathrm{H}_{2} \mathrm{O}$ & {$[150]$} \\
\hline $7-\operatorname{CoS} / \mathrm{C}$ & Heat treatment \& sulfur & $\mathrm{Co}\left(\mathrm{NO}_{3}\right)_{2} \bullet 6 \mathrm{H}_{2} \mathrm{O}$ & [151] \\
\hline MWCNTs $/ \mathrm{Co}_{9} \mathrm{~S}_{8}$ composites & Solvothermal \& heat treatment & $\mathrm{Co}\left(\mathrm{NO}_{3}\right)_{2} \bullet 6 \mathrm{H}_{2} \mathrm{O}$ & [152] \\
\hline $\mathrm{Co}_{9} \mathrm{~S}_{8} / \mathrm{Co}$ & Ball-milling & Co powder & [153] \\
\hline $\mathrm{Co}_{9} \mathrm{~S}_{8} @ \mathrm{CNNs}$ & Freeze-drying \& heat treatment & $\mathrm{CoCl}_{2} \bullet 6 \mathrm{H}_{2} \mathrm{O}$ & [154] \\
\hline $\mathrm{CoS}_{2} / \mathrm{NCNTF}$ & Heat treatment \& sulfur & $\mathrm{Co}\left(\mathrm{NO}_{3}\right)_{2} \bullet 6 \mathrm{H}_{2} \mathrm{O}$ & {$[155]$} \\
\hline $\mathrm{Co}_{9} \mathrm{~S}_{8} / \mathrm{N}-\mathrm{C}$ hollow nanospheres & Heat treatment \& sulfur & $\mathrm{CoSO}_{4} \bullet 7 \mathrm{H}_{2} \mathrm{O}$ & {$[28]$} \\
\hline $\mathrm{Co}_{9} \mathrm{~S}_{8} / \mathrm{RGO}$ & Hydrothermal $\left(180^{\circ} \mathrm{C}, 12 \mathrm{~h}\right)$ & $\left(\mathrm{C}_{2} \mathrm{H}_{3} \mathrm{O}_{2}\right)_{2} \mathrm{Co} \bullet 4 \mathrm{H}_{2} \mathrm{O}$ & [97] \\
\hline $\mathrm{CoS}_{2} / \mathrm{G}$ composite & Hydrothermal $\left(200^{\circ} \mathrm{C}, 12 \mathrm{~h}\right)$ & $\left(\mathrm{C}_{2} \mathrm{H}_{3} \mathrm{O}_{2}\right)_{2} \mathrm{Co} \bullet 4 \mathrm{H}_{2} \mathrm{O}$ & [156] \\
\hline $\mathrm{CoS}_{2} @ \mathrm{MCNF}$ & Hydrothermal \& heat treatment & $\mathrm{CoCl}_{2} \bullet 6 \mathrm{H}_{2} \mathrm{O}$ & {$[157]$} \\
\hline $\begin{array}{c}\mathrm{Ni}_{3} \mathrm{~S}_{2} / \mathrm{Co}_{9} \mathrm{~S}_{8} / \mathrm{N} \text {-doped carbon } \\
\text { composite }\end{array}$ & Hydrothermal \& heat treatment & $\mathrm{Co}\left(\mathrm{NO}_{3}\right)_{2} \bullet 6 \mathrm{H}_{2} \mathrm{O}$ & [158] \\
\hline CoSe/Co@NC & Heat treatment $\left(800^{\circ} \mathrm{C}, \mathrm{Ar}\right)$ & $\mathrm{Co}\left(\mathrm{NO}_{3}\right)_{2} \bullet 6 \mathrm{H}_{2} \mathrm{O}$ & [159] \\
\hline $\mathrm{Co}_{0.85} \mathrm{Se} \mathrm{NSs} / \mathrm{G}$ & Hydrothermal $\left(180^{\circ} \mathrm{C}, 16 \mathrm{~h}\right)$ & $\left(\mathrm{C}_{2} \mathrm{H}_{3} \mathrm{O}_{2}\right)_{2} \mathrm{Co} \bullet 4 \mathrm{H}_{2} \mathrm{O}$ & {$[160]$} \\
\hline Co-Zn-Se@C & Hydrothermal \& heat treatment & $\mathrm{Co}\left(\mathrm{NO}_{3}\right)_{2} \bullet 6 \mathrm{H}_{2} \mathrm{O}$ & {$[161]$} \\
\hline $\mathrm{CoSe}_{2} @ \mathrm{NC}-\mathrm{NR} / \mathrm{CNT}$ & Selenization & $\mathrm{Co}\left(\mathrm{NO}_{3}\right)_{2} \bullet 6 \mathrm{H}_{2} \mathrm{O}$ & [162] \\
\hline $\begin{array}{c}\text { cobblestone-like CoSe } e_{2} @ \mathrm{C} \\
\text { nanospheres }\end{array}$ & Heat treatment $\left(500{ }^{\circ} \mathrm{C}, \mathrm{Ar} / \mathrm{H}_{2}\right)$ & $\mathrm{Co}\left(\mathrm{NO}_{3}\right)_{2} \bullet 6 \mathrm{H}_{2} \mathrm{O}$ & [32] \\
\hline Cu-doped $\mathrm{CoSe}_{2}$ microboxes & Hydrothermal $\left(160^{\circ} \mathrm{C}, 8 \mathrm{~h}\right)$ & $\left(\mathrm{C}_{2} \mathrm{H}_{3} \mathrm{O}_{2}\right)_{2} \mathrm{Co} \bullet 4 \mathrm{H}_{2} \mathrm{O}$ & [163] \\
\hline $\mathrm{CoSe}_{2} / \mathrm{N}-\mathrm{CNFs}$ & Electrospinning \& heat treatment & $\left(\mathrm{C}_{2} \mathrm{H}_{3} \mathrm{O}_{2}\right)_{2} \mathrm{Co} \bullet 4 \mathrm{H}_{2} \mathrm{O}$ & [24] \\
\hline $\mathrm{CoSe}_{2} / \mathrm{C}-\mathrm{ND} @ \mathrm{RGO}$ & Heat treatment $\left(600{ }^{\circ} \mathrm{C}\right.$, Air $)$ & $\mathrm{Co}\left(\mathrm{NO}_{3}\right)_{2} \bullet 6 \mathrm{H}_{2} \mathrm{O}$ & [164] \\
\hline CoSe@CSs & Hydrothermal \& heat treatment & $\mathrm{Co}\left(\mathrm{NO}_{3}\right)_{2} \bullet 6 \mathrm{H}_{2} \mathrm{O}$ & [60] \\
\hline CoSe quasi-microspheres & Hydrothermal $\left(180^{\circ} \mathrm{C}, 2 \mathrm{~h}\right)$ & $\mathrm{Co}\left(\mathrm{NO}_{3}\right)_{2} \bullet 6 \mathrm{H}_{2} \mathrm{O}$ & {$[165]$} \\
\hline yolk-shell structured CoSe/C & Heat treatment $\left(800^{\circ} \mathrm{C}, \mathrm{Ar}\right)$ & $\mathrm{Co}\left(\mathrm{NO}_{3}\right)_{2} \bullet 6 \mathrm{H}_{2} \mathrm{O}$ & {$[166]$} \\
\hline $\mathrm{Co}_{9} \mathrm{Se}_{8} / \mathrm{RGO}$ hybrid nanosheet & Hydrothermal $\left(180^{\circ} \mathrm{C}, 12 \mathrm{~h}\right)$ & $\left(\mathrm{C}_{2} \mathrm{H}_{3} \mathrm{O}_{2}\right)_{2} \mathrm{Co} \bullet 4 \mathrm{H}_{2} \mathrm{O}$ & [97] \\
\hline urchin-like $\mathrm{CoSe}_{2}$ nanorods & Electrospinning \& heat treatment & $\left(\mathrm{C}_{2} \mathrm{H}_{3} \mathrm{O}_{2}\right)_{2} \mathrm{Co} \bullet 4 \mathrm{H}_{2} \mathrm{O}$ & [93] \\
\hline $\mathrm{CoSe}_{2} @ \mathrm{C} / \mathrm{CNTs}$ & Heat treatment \& selenization & $\mathrm{Co}\left(\mathrm{NO}_{3}\right)_{2} \bullet 6 \mathrm{H}_{2} \mathrm{O}$ & [48] \\
\hline $\mathrm{CoSe}_{2} @ \mathrm{~N}-\mathrm{PGC} / \mathrm{CNTs}$ & Heat treatment \& selenization & $\mathrm{Co}\left(\mathrm{NO}_{3}\right)_{2} \bullet 6 \mathrm{H}_{2} \mathrm{O}$ & [167] \\
\hline $\mathrm{Co} /(\mathrm{NiCo}) \mathrm{Se}_{2}$ box in box structure & Selenization $\left(270{ }^{\circ} \mathrm{C} 6 \mathrm{~h}\right)$ & $\mathrm{Co}\left(\mathrm{NO}_{3}\right)_{2} \bullet 6 \mathrm{H}_{2} \mathrm{O}$ & {$[71]$} \\
\hline $\mathrm{CoSe}_{2}$ powders & Hydrothermal $\left(180^{\circ} \mathrm{C}, 18 \mathrm{~h}\right)$ & $\mathrm{Co}\left(\mathrm{NO}_{3}\right)_{2} \bullet 6 \mathrm{H}_{2} \mathrm{O}$ & [168] \\
\hline $\mathrm{CoSe}_{2}$ nanoparticles & Hydrothermal $\left(180^{\circ} \mathrm{C}, 24 \mathrm{~h}\right)$ & $\mathrm{CoCl}_{2} \bullet 6 \mathrm{H}_{2} \mathrm{O}$ & [169] \\
\hline $\mathrm{CoSe}_{2}$ microspheres & Heat treatment & $\mathrm{Co}\left(\mathrm{NO}_{3}\right)_{2} \bullet 6 \mathrm{H}_{2} \mathrm{O}$ & [170] \\
\hline CoSe@PCP & Heat treatment \& selenization & $\mathrm{Co}\left(\mathrm{NO}_{3}\right)_{2} \bullet 6 \mathrm{H}_{2} \mathrm{O}$ & {$[68]$} \\
\hline
\end{tabular}

\section{Application of Cobalt-Based Anode Materials in LIBs/SIBs}

\subsection{Cobalt-Based Alloys and Its Composites}

As mentioned above, the electrochemical properties of cobalt-based alloys are mostly decided by metal components alloyed with cobalt, owing to the inactive nature with $\mathrm{Li} / \mathrm{Na}$ of the cobalt ion. 
As an inactive component of cobalt-based alloys, the presence of phase cobalt can alleviate the volume fluctuation as a protective matrix, thereby improving the electrochemical performances of cobalt-based alloys. Despite these features, the electrochemical properties of cobalt-based alloys are still disturbed by the pulverization and shedding of active materials during periodical cycles. Many cobalt-based alloys have been reported as anode materials for LIBs or SIBs with outstanding electrochemical properties owing to their unique structures and suitable compositions [40,55,67,81,83,92,104,105,171].

Shi et al. reported a novel structure with a Sn-Co alloy embedded in porous N-doped carbon microboxes that exhibited a superior electrochemical performance when used as anode materials for LIBs [64]. In Figure 9a, the Sn-Co@C-2 electrode exhibited the highest specific capacity at various current densities, from 0.1 to $2 \mathrm{~A} \mathrm{~g} \mathrm{~g}^{-1}$, indicating a superior rate performance. Furthermore, the Sn-Co@C-2 electrode delivered outstanding capacity retention when the current density went back to $0.1 \mathrm{~A} \mathrm{~g}^{-1}$. Ex-situ XRD of the Sn-Co@C-2 electrode before and after cycles are shown in Figure 9b. CoSn2 disappeared after the first cycle, and the CoSn phase maintained well even after 100 cycles, suggesting a good reversibility of the Sn-Co@C-2 electrode.
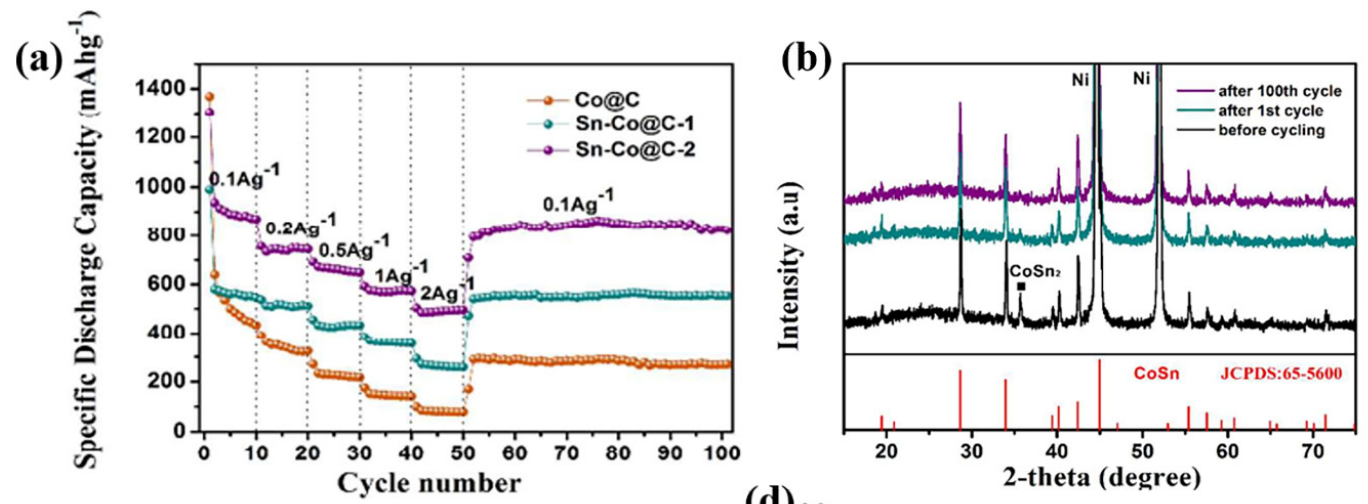

(c)
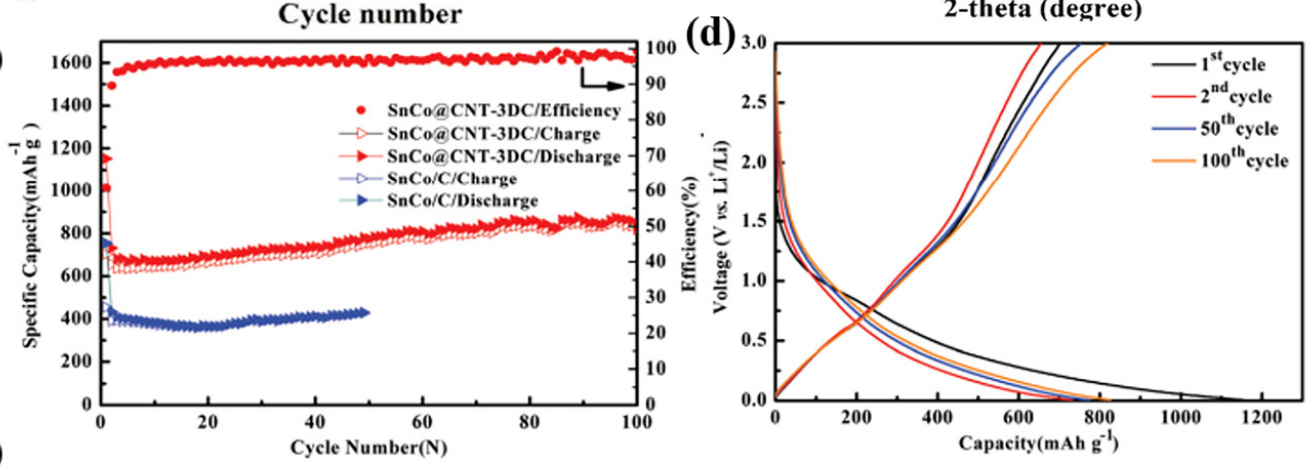

(e)
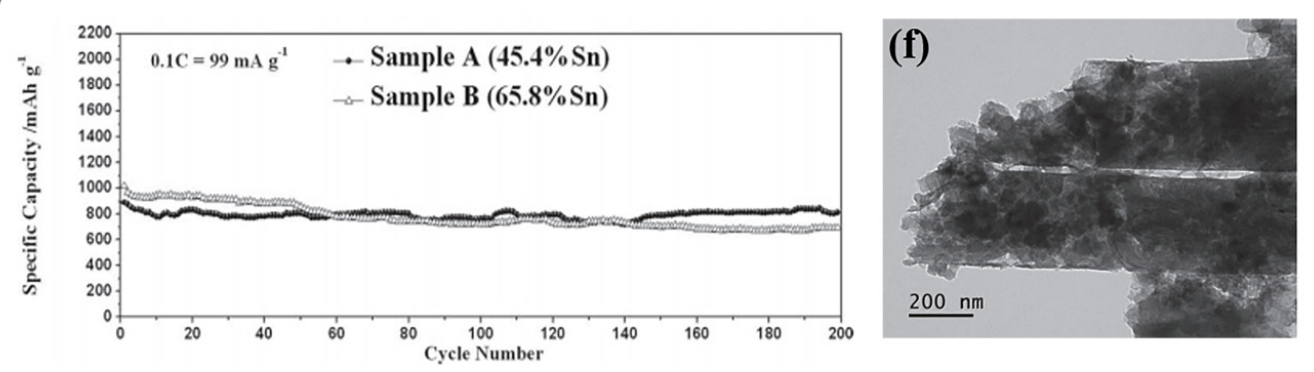

Figure 9. Electrochemical performances of cobalt-based anode materials. (a) Rate performances of Co@C, Sn-Co@C-1 and Sn-Co@C-2 as anode materials of LIBs. (b) XRD patterns of the Sn-Co@C-2 electrode before and after the charge-discharge cycles [64]. (c) Cycle performance and (d) charge/discharge profiles of the SnCo@CNT-3DC electrode at $50 \mathrm{~mA} \mathrm{~g}^{-1}$ in the voltage range of $0.005-3 \mathrm{~V} \mathrm{vs.} \mathrm{Li}^{-\mathrm{Li}^{+}}$[44]. (e) Cycle performance of the Sn-Co-CNT@CNT nanostructure tested at $0.1 \mathrm{C}\left(1 \mathrm{C}=990 \mathrm{~mA} \mathrm{~g}^{-1}\right)$. (f) TEM image of the Sn-Co-CNT@CNT anode materials after 200 cycles at 1C in LIBs [67]. 
He's group reported a novel method for the one-step synthesis of hierarchical SnCo@CNT-3DC [44]. When used as anode materials for LIBs, this unique structure can provide numerous channels for electron transfer, shorten the diffusion pathway of $\mathrm{Li}^{+}$and ensure the permeation of electrolytes. As shown in Figure 9c, the SnCo@CNT-3DC electrode displayed a high capacity of $826 \mathrm{~mA} \mathrm{~h} \mathrm{~g}{ }^{-1}$ after 100 cycles at $0.1 \mathrm{~A} \mathrm{~g}^{-1}$. Compared with the first discharge capacity, the capacity retention rate is $113 \%$. During the cycles, there is a slight increase in capacity owing to the increasing electrochemical reaction of the SnCo nanoparticles caused by the activation of the electrode materials. As for the $\mathrm{SnCo} / \mathrm{C}$ electrode, the relatively low capacity of $429.6 \mathrm{~mA} \mathrm{~h} \mathrm{~g}^{-1}$ was retained after 50 cycles at $0.1 \mathrm{~A} \mathrm{~g}^{-1}$. These results can be probably ascribed to the better transformation of lithium in the SnCo@CNT-3DC electrode. Figure 9d shows that the SnCo@CNT-3DC electrode delivered a high discharge capacity of $1154.4 \mathrm{~mA} \mathrm{~h} \mathrm{~g}^{-1}$, with an initial coulombic efficiency (CE) of $61 \%$. As the number of cycle increases, the capacity decreases slightly, suggesting good capacity retention.

Wang's group demonstrated an in-situ template technique for the preparation of the Sn-Co-CNT@CNT ternary tube-in-tube nanostructure [67]. When utilized as anode materials for LIBs, superior electrochemical properties were obtained owing to the confined volume change in the nanotube cavities and ensured a permanent electrical connectivity of the immobilized Sn-Co anodes. As shown in Figure 9e, the Sn-Co-CNT@CNT electrode with 45.4\% Sn delivered a discharge capacity of $811 \mathrm{~mA} \mathrm{~h} \mathrm{~g}^{-1}$ after 200 cycles, with $91.1 \%$ of the initial discharge capacity retained. The TEM image of the Sn-Co-CNT@CNT anode after 200 cycles demonstrates that the 1D nanotube-like structure was retained, indicating its structural stability during the cycles (Figure 9f).

\subsection{Cobalt Oxides and Its Composites}

Cobalt oxides have been identified as hopeful anode materials for LIBs and SIBs due to their high theoretical capacities, natural abundances, and low costs. For instance, $\mathrm{Co}_{3} \mathrm{O}_{4}$, as the most common cobalt oxide, has a theoretical capacity of $890 \mathrm{~mA} \mathrm{~h} \mathrm{~g}^{-1}$ attributed to its eight-electron transfer reaction during cycling. However, the intrinsic defects of cobalt oxides, including low structural stability and poor electrical conductivity, lead to inferior electrochemical properties $[57,63,77,78,111,113,116,118,120]$. Yu's group reported hierarchically structured $\mathrm{Co}_{3} \mathrm{O}_{4} @$ carbon porous fibers to circumvent the volume fluctuation issue during lithiation/delithiation [11]. As shown in Figure 10a, the initial discharge and charge specific capacity of ES- $\mathrm{CNCO}_{3} \mathrm{O}_{4}$ was 1824 and $1003 \mathrm{~mA} \mathrm{~h} \mathrm{~g}^{-1}$, with an initial columbic efficiency of $55 \%$. After five cycles, the charge/discharge profiles were almost overlapped, indicating the high stability of the composite. 
(a)

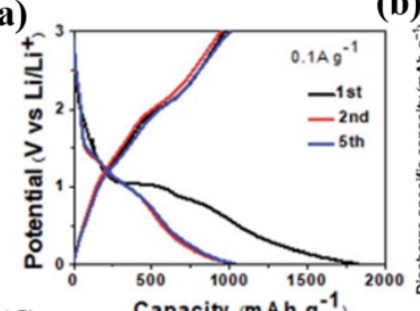

(d)
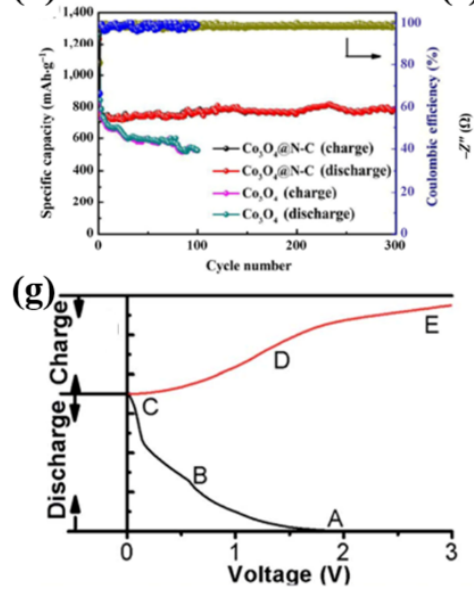

(b)

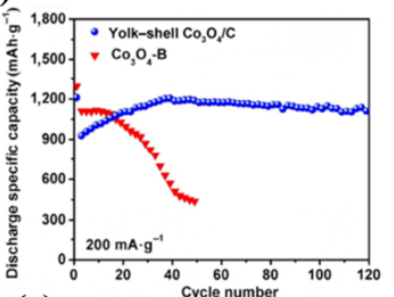

(e)

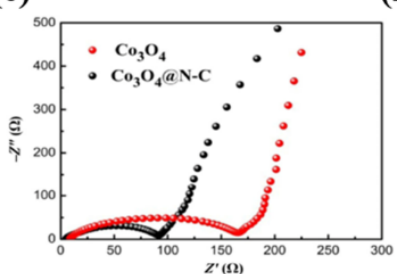

(h) (c)

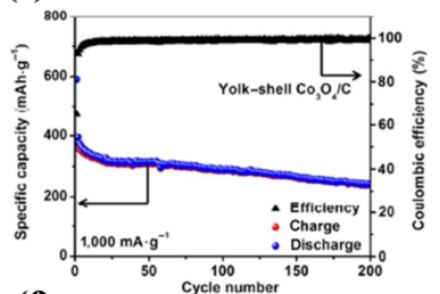

(f)
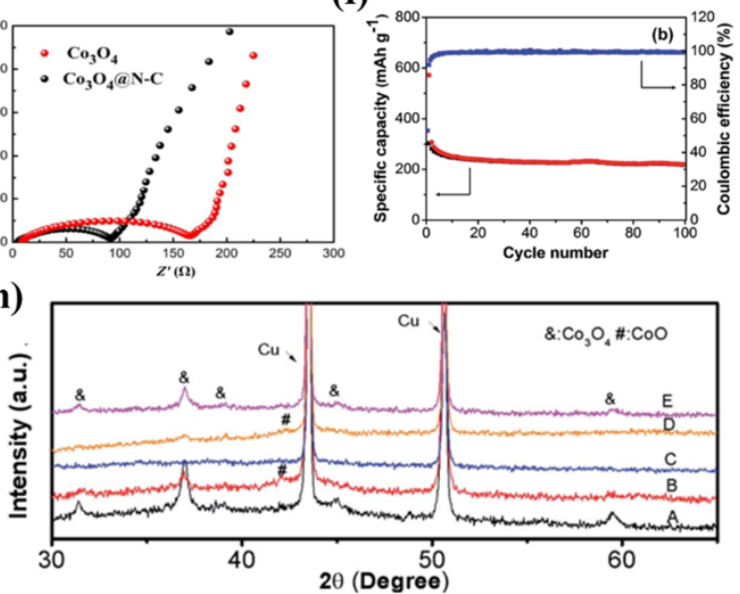

Figure 10. Electrochemical performances of cobalt-based anode materials. (a) Galvanostatic discharge-charge profiles of ES- $\mathrm{CNCO}_{3} \mathrm{O}_{4}$ at $0.1 \mathrm{~A} \mathrm{~g}^{-1}$ [11]. (b) Cycling performance of the yolk-shell $\mathrm{Co}_{3} \mathrm{O}_{4}$ and $\mathrm{Co}_{3} \mathrm{O}_{4}$-B at $0.2 \mathrm{~A} \mathrm{~g}^{-1}$ for LIBs. (c) Cycling properties of the yolk-shell $\mathrm{Co}_{3} \mathrm{O}_{4}$ at $1 \mathrm{~A} \mathrm{~g}^{-1}$ for SIBs [129]. (d) Cycling performance of $\mathrm{Co}_{3} \mathrm{O}_{4}$ and $\mathrm{Co}_{3} \mathrm{O}_{4} @ \mathrm{~N}-\mathrm{C}$ at $0.5 \mathrm{~A} \mathrm{~g}^{-1}$. (e) Electrochemical impedance spectra for $\mathrm{Co}_{3} \mathrm{O}_{4}$ and $\mathrm{Co}_{3} \mathrm{O}_{4} @ \mathrm{~N}-\mathrm{C}$ [128]. (f) Cycling performance of the Ni-doped $\mathrm{Co} / \mathrm{CoO} / \mathrm{NC}$ electrode and the corresponding columbic efficiencies at $0.5 \mathrm{~A} \mathrm{~g}^{-1}$ [126]. (g) Galvanostatic charge-discharge profiles. Points A, B, C, D and E represent the pristine state, the discharge state at $0.6 \mathrm{~V}$ and $0.01 \mathrm{~V}$ and the charged state at $1.4 \mathrm{~V}$ and $3 \mathrm{~V}$, respectively. (h) Ex-situ XRD patterns corresponding to these points [124].

Wu et al. synthesized yolk-shell $\mathrm{Co}_{3} \mathrm{O}_{4} / \mathrm{C}$ dodecahedrons to solve the problem of volume change during lithiation or sodiation [129]. When utilized as an anode for LIBs, the yolk-shell $\mathrm{Co}_{3} \mathrm{O}_{4} / \mathrm{C}$ electrode delivered a high discharge capacity of $1100 \mathrm{~mA} \mathrm{~h} \mathrm{~g}{ }^{-1}$ after 120 cycles. Regarding $\mathrm{Co}_{3} \mathrm{O}_{4}-\mathrm{B}$, a lower specific capacity of $400 \mathrm{~mA} \mathrm{~h} \mathrm{~g}^{-1}$ after 50 cycles was obtained, suggesting a better lithium storage performance of yolk-shell $\mathrm{Co}_{3} \mathrm{O}_{4} / \mathrm{C}$. Figure 10c shows the sodium storage performance of yolk-shell $\mathrm{Co}_{3} \mathrm{O}_{4} / \mathrm{C}$ at a current density of $1 \mathrm{~A} \mathrm{~g}^{-1}$. The second discharge capacity of the sample was $395 \mathrm{~mA} \mathrm{~h} \mathrm{~g}^{-1}$, and the specific capacity of $240 \mathrm{~mA} \mathrm{~h} \mathrm{~g}^{-1}$ was obtained after 200 cycles, suggesting the great electrochemical properties of yolk-shell $\mathrm{Co}_{3} \mathrm{O}_{4} / \mathrm{C}$ in SIBs.

Xie's group reported a porous starfish-like nanocomposite of $\mathrm{N}$-doped carbon with $\mathrm{Co}_{3} \mathrm{O}_{4}$ coating [128]. The $\mathrm{Co}_{3} \mathrm{O}_{4} @ \mathrm{~N}-\mathrm{C}$ electrode as a LIB anode delivered an initial specific capacity of $1235 \mathrm{~mA} \mathrm{~h} \mathrm{~g}^{-1}$ and a capacity of $795 \mathrm{~mA} \mathrm{~h} \mathrm{~g}^{-1}$ after 300 cycles, indicating a superior lithium storage performance to pure $\mathrm{Co}_{3} \mathrm{O}_{4}$ (Figure 10d). To better understand the gap between the electrochemical performances of the two samples, Nyquist plots of $\mathrm{Co}_{3} \mathrm{O}_{4} @ \mathrm{~N}-\mathrm{C}$ and pure $\mathrm{Co}_{3} \mathrm{O}_{4}$ electrodes were fitted (Figure 10e). It is clear that the $\mathrm{Co}_{3} \mathrm{O}_{4} @ \mathrm{~N}-\mathrm{C}$ composite exhibited smaller charge-transfer resistance, suggesting that the porous structure accelerates the diffusion of $\mathrm{Li}^{+}$, and the presence of $\mathrm{N}$-doped carbon improves the electrical conductivity of the sample, finally resulting in the superior electrochemical properties of $\mathrm{Co}_{3} \mathrm{O}_{4} @ \mathrm{~N}-\mathrm{C}$. Yang's group reported the preparation of a $\mathrm{Ni}$-doped $\mathrm{Co} / \mathrm{CoO} / \mathrm{N}-\mathrm{doped}$ carbon hybrid, with Ni-Co-ZIF as the precursor [126]. When utilized as an anode material for SIBs, the $\mathrm{Ni}$-doped $\mathrm{Co} / \mathrm{CoO} / \mathrm{NC}$ electrode exhibited a high discharge capacity of $218.7 \mathrm{~mA} \mathrm{~h} \mathrm{~g}^{-1}$ after 100 cycles, with $87.5 \%$ capacity retained (Figure 10f). 
Wang and his collogues reported a nanocomposite of $\mathrm{N}$-doped carbon with $\mathrm{Co}_{3} \mathrm{O}_{4}$ nanoparticles, exhibiting a superior sodium storage performance [124]. To further investigate the electrochemical mechanism of the electrode, five points that appeared at the first charge-discharge profile were tested by ex-situ XRD patterns (Figure 10g,h). Upon the discharge process, the phase of $\mathrm{Co}_{3} \mathrm{O}_{4}$ gradually disappeared along with the sodiation. When the cell discharged to $0.6 \mathrm{~V}(\mathrm{~B})$, a new phase of $\mathrm{CoO}$ came out owing to the reduction of $\mathrm{Co}_{3} \mathrm{O}_{4}$. When fully discharged (C), only one phase of the $\mathrm{Cu}$ collector was retained, attributed to the poor crystallization of the $\mathrm{Na}_{2} \mathrm{O}$ phase. During the following charge process, reverse reactions occurred accompanied by the reappearance of $\mathrm{CoO}$ and $\mathrm{Co}_{3} \mathrm{O}_{4}$ at $1.4 \mathrm{~V}$ and only $\mathrm{Co}_{3} \mathrm{O}_{4}$ when fully charged $(\mathrm{E})$. These results can be attributed to a reversible reaction of $\mathrm{Co}_{3} \mathrm{O}_{4}+8 \mathrm{Na}^{+}+8 \mathrm{e}^{-} \leftrightarrow 3 \mathrm{Co}+4 \mathrm{Na}_{2} \mathrm{O}$ during cycling.

\subsection{Cobalt Sulfide and Its Composites}

Cobalt sulfide has been widely studied as an anode material for LIBs/SIBs owing to its high theoretical capacity, satisfactory electrical conductivities and fair thermal stabilities. Similar to cobalt oxides, the main obstacle to the development of cobalt sulfide is the severe volume fluctuation during periodical cycling $[14,16,17,26,36,47,49,66,172-186]$. He and his colleagues synthesized self-assembled $\mathrm{CoS}_{2}$ nanoparticles wrapped by $\mathrm{CoS}_{2}$-quantum-dots-anchored graphene nanosheets (denoted as $\mathrm{CoS}_{2}$ NP@G-CoS 2 QD) and tested as an anode material for LIBs [187]. As displayed in Figure 11a, the $\mathrm{CoS}_{2}$ NP@G-CoS 2 QD delivered a high specific capacity of $831 \mathrm{~mA} \mathrm{~h} \mathrm{~g}^{-1}$ at $1 \mathrm{~A} \mathrm{~g}^{-1}$ after 300 cycles. Meanwhile, the nearly $100 \%$ coulombic efficiency over all 300 cycles also indicates the excellent cycle performance of this material. The enhanced cycle performance can be ascribed to the presence of few-layer graphene, which can not only improve the electrical conductivity of this composite but also alleviate the volume fluctuation and aggregation of $\operatorname{CoS}_{2}$ (Figure 11b).

Sun's group synthesized a sponge-like composite assembled with $\mathrm{Co}_{9} \mathrm{~S}_{8}$ quantum dots embedded in a carbon matrix, which was wrapped by rGO [172]. When utilized as an anode material for SIBs, the reaction mechanism was studied by an ex-situ XRD pattern in Figure 11c. Upon sodiation, the peaks of $\mathrm{Co}_{9} \mathrm{~S}_{8}$ gradually disappeared until the discharge was completed. At the same time, the peaks of the $\mathrm{Na}_{2} \mathrm{~S}$ and $\mathrm{Co}$ phases increasingly appeared owing to the reduction reaction from $\mathrm{Co}_{9} \mathrm{~S}_{8}$ to $\mathrm{Co}$ and $\mathrm{Na}_{2} \mathrm{~S}$. During the subsequent charge process, an inverse reaction, including the disappearance of the $\mathrm{Na}_{2} \mathrm{~S}$ and $\mathrm{Co}$ phases and the reappearance of $\mathrm{Co}_{9} \mathrm{~S}_{8}$, occurred. This situation indicates the high reversibility of $\mathrm{Co}_{9} \mathrm{~S}_{8}$ quantum dots during the initial charge-discharge process. Peng's group built a CoS@rGO composite assembled by CoS nanoplates decorated on rGO and tested as an anode material for SIBs [173]. To investigate the industry application of this composite, a full cell was fabricated with $\mathrm{Na}_{3} \mathrm{VPO}_{4} @ \mathrm{C}$ nanowires as the cathode (Figure 11d). As shown in Figure 11e, the reversible capacity of $290 \mathrm{~mA} \mathrm{~h} \mathrm{~g}^{-1}$ was obtained after 100 cycles, suggesting the superior cycle performance of a CoS@rGO// $\mathrm{Na}_{3} \mathrm{VPO}_{4}$ (NVP)@C full battery. The alight red LED (inset of Figure 11e) fully demonstrates the practical application of a CoS@rGO//NVP@C full battery. This excellent electrochemical performance resulted from the novel structure and the presence of rGO.

Lou's group reported a $\mathrm{CoS}_{2}$ nanobubble hollow prism and tested it as an anode material for LIBs [174]. This sample delivered remarkable specific capacities of 910, 778, 681 and $470 \mathrm{~mA} \mathrm{~h} \mathrm{~g}^{-1}$ at the current densities of 200,500, 1000 and $5000 \mathrm{~mA} \mathrm{~g}^{-1}$, respectively (Figure 11f). It is worth mentioning that the capacity recovered to $864 \mathrm{~mA} \mathrm{~h} \mathrm{~g}^{-1}$ when the current density was returned to $200 \mathrm{~mA} \mathrm{~g}^{-1}$, indicating the outstanding robustness of the electrode. Qian's group synthesized hollow nanospheres assembled of mesoporous $\mathrm{Cog}_{9} \mathrm{~S}_{8}$ [175]. When utilized as an anode material of LIBs, an excellent specific capacity was maintained as $896 \mathrm{~mA} \mathrm{~h} \mathrm{~g}^{-1}$ even after 800 cycles at $2 \mathrm{~A} \mathrm{~g}^{-1}$ (Figure 11g). The superior cycle performance resulted from the remarkable charge-transfer kinetics. Furthermore, Zhou and coworkers prepared a one-dimensional multiwalled carbon nanotube@a-C@Co $9 \mathrm{~S}_{8}$ nanocomposites (MWCNT@a-C@Co $9 \mathrm{~S}_{8}$ ) as an anode material for advanced LIBs [5]. In Figure 11h, a high reversible capacity of $1065 \mathrm{~mA} \mathrm{~h} \mathrm{~g}^{-1}$ was obtained after 700 cycles at a current density of $2 \mathrm{~A} \mathrm{~g}^{-1}$. It is worth 
mentioning that a slight fluctuation was observed during the cycling, ascribed to the enhanced surface lithium storage, the electrode reactivation and so on.
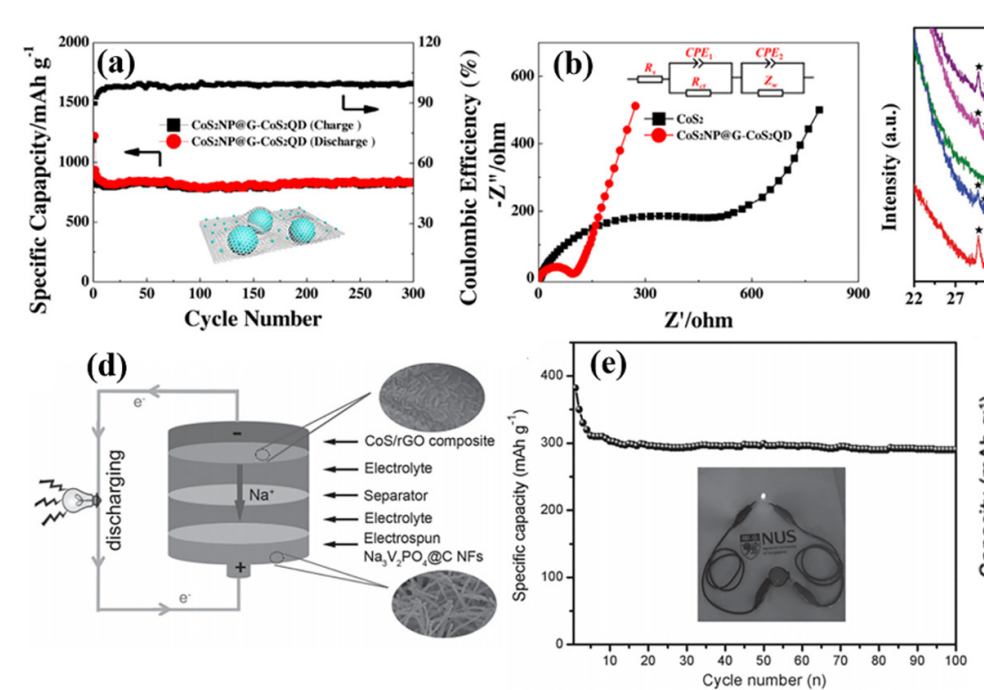
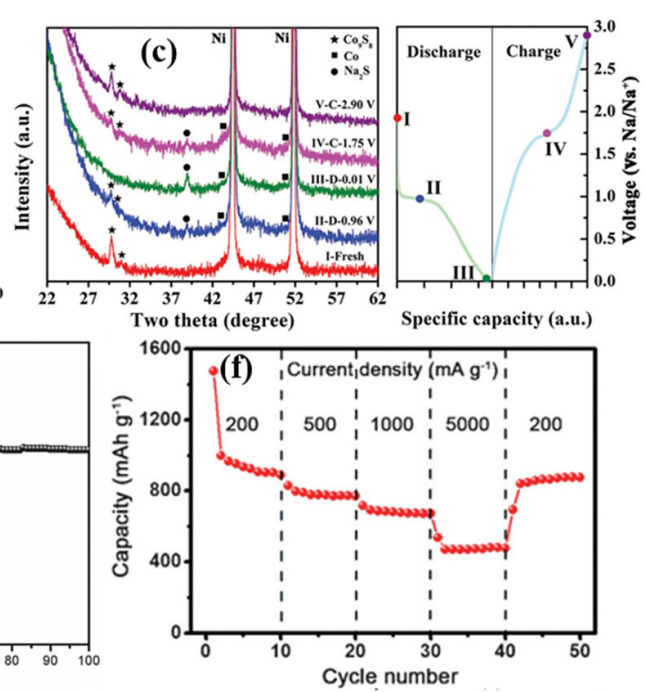
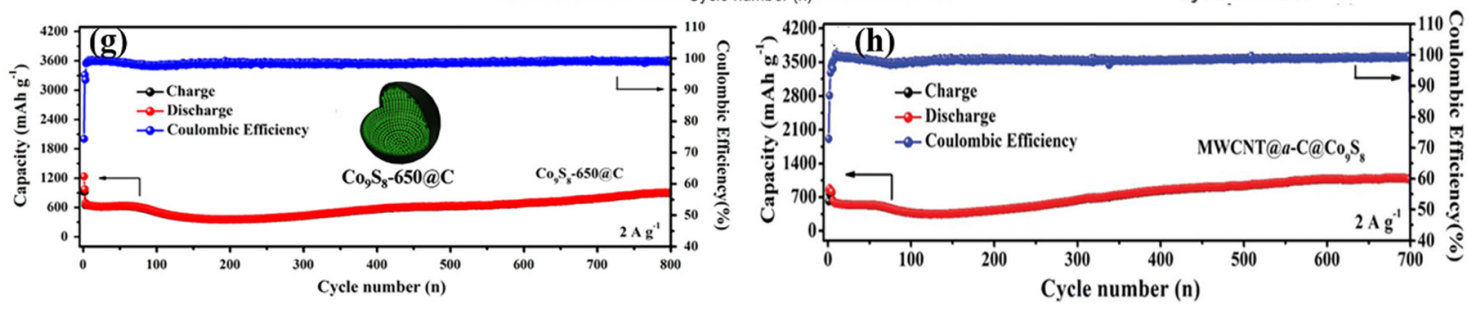

Figure 11. Electrochemical performances of cobalt-based anode materials. (a) Cycle performance of $\mathrm{CoS}_{2}$-quantum-dots-anchored graphene nanosheets $\left(\mathrm{CoS}_{2} \mathrm{NP@G-CoS} 2 \mathrm{QD}\right)$ at $1 \mathrm{~A} \mathrm{~g}^{-1}$. (b) Nyquist plots of $\mathrm{CoS}_{2}$ and $\mathrm{CoS}_{2}$ NP@G-CoS 2 QD electrodes after 300 cycles [187]. (c) Ex-situ XRD of the cobalt sulfides quantum dots@mesoporous hollow carbon polyhedral@reduced graphene oxide $\left(\mathrm{Co}_{9} \mathrm{~S}_{8}\right.$ QD@HCP)@rGO electrode tested at different voltage states [172]. (d) Schematic illustration and (e) cycle performance of CoS@rGO// $\mathrm{Na}_{3} \mathrm{VPO}_{4}$ (NVP)@C sodium ion full cell [173]. (f) Rate performance of $\mathrm{CoS}_{2}$ bubble-like hollow prisms [174]. (g) Cycle performance of $\mathrm{Co}_{9} \mathrm{~S}_{8}-650 @ \mathrm{C}$ in LIBs [175]. (h) Cycle performance of Multiwalled carbon nanotube@a-C@Cog $\mathrm{S}_{8}$ nanocomposites (MWCNT@a-C@Co $9 \mathrm{~S}_{8}$ ) anode for LIBs [5].

\subsection{Cobalt Phosphide and Its Composites}

Cobalt phosphides, as promising electrode materials, have been studied as anode materials for LIBs/SIBs. Compared with cobalt oxides and sulfides, cobalt phosphides possess higher gravimetric/volumetric specific capacities. The main drawbacks that restrict their applications are the severe volume expansion during lithiation or sodiation and poor electrical conductivities. Many efforts have been made to circumvent these issues $[18,31,46,82,134,136,137,188,189]$. Sun's group hybridized the $\mathrm{Co}_{2} \mathrm{P}$ nanoparticles with $\mathrm{N}$-doped carbon matrices and tested the composite as an anode material for SIBs [143]. Except for the initial discharge profile, the profiles of the galvanostatic charge-discharge process overlapped well with each other, indicating a good reversibility of $\mathrm{Co}_{2} \mathrm{P} @ \mathrm{~N}-\mathrm{C} @ \mathrm{rGO}$ (Figure 12a). Yan's group successfully fabricated $\mathrm{Co}_{x} \mathrm{P}$ nanostructures with controlled phases, sizes and shapes. When applied as the anode material of LIBs, the hollow CoP particles delivered a capacity of $630 \mathrm{~mA}$ $\mathrm{h} \mathrm{g}^{-1}$ after 100 cycles, as shown in Figure 12b. With regard to solid CoP nanoparticles, a capacity of $480 \mathrm{~mA} \mathrm{~h} \mathrm{~g}^{-1}$ was retained after 100 cycles. Better cycling performance of the hollow CoP electrode can be ascribed to the hollow structure, which ensures effective lithiation/delithiation and alleviates the volume change during periodical cycling. 

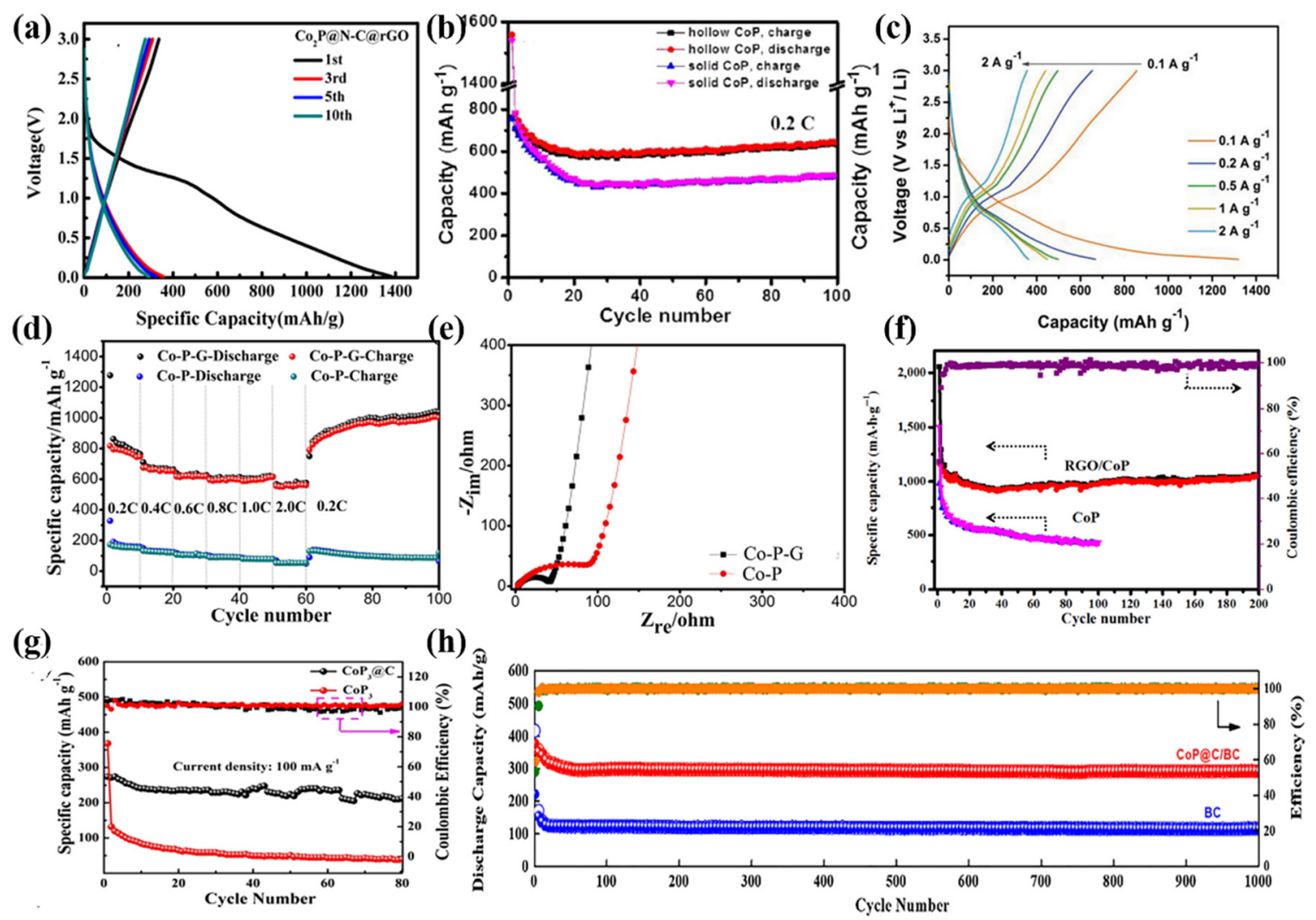

Figure 12. Electrochemical performances of cobalt-based anode materials. (a) Charge-discharge profiles of $\mathrm{Co}_{2} \mathrm{P} @ \mathrm{~N}$-doped $\mathrm{C}(\mathrm{N}-\mathrm{C}) @$ reduced graphene oxide (rGO) in various cycles [143]. (b) Cycle performances of CoP hollow and solid nanoparticles [79]. (c) Charge-discharge profiles of N, P co-doped

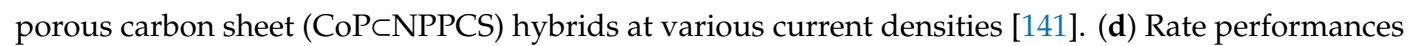
and (e) EIS spectra of $\mathrm{Co}_{2} \mathrm{P}-\mathrm{Co} /$ graphene and $\mathrm{Co}_{2} \mathrm{P}-\mathrm{Co}$ composites [139]. (f) Cycle performances of $\mathrm{CoP} / \mathrm{RGO}$ and $\mathrm{CoP}$ [135]. (g) Cycle performances of $\mathrm{CoP}_{3} @ \mathrm{C}$ and $\mathrm{CoP}_{3}$ [80]. (h) Cycling performance of CoP@C/biomass-derived carbon (BC) and pure BC [142].

Xiong's group firstly synthesized a novel composite with core-like CoP embedded in N, P co-doped

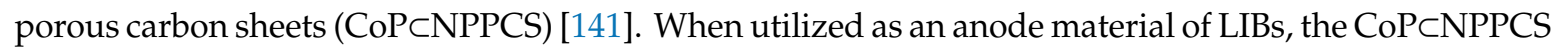
electrode delivered reversible capacities of about 709,500,501, 410 and $357 \mathrm{~mA} \mathrm{~h} \mathrm{~g}^{-1}$ at the current densities of $0.1,0.2,0.5$ and $1.0 \mathrm{~A} \mathrm{~g}^{-1}$, respectively. It is worth noting that the capacity retention was over $50 \%$ when the current density was expanded by 20 times, indicating the excellent rate capability. Peng's group prepared $\mathrm{Co}_{2} \mathrm{P}-\mathrm{Co}(\mathrm{Co}-\mathrm{P})$ hollow nanospheres decorated with graphene sheets and tested them as anode materials for LIBs [139]. When the current densities increased from $0.2 \mathrm{C}, 0.4 \mathrm{C}$, $0.6 \mathrm{C}, 0.8 \mathrm{C}$ and $1.0 \mathrm{C}$ to $2.0 \mathrm{C}\left(1 \mathrm{C}=540 \mathrm{~mA} \mathrm{~g}^{-1}\right)$, the capacity of Co-P/graphene correspondingly decreased from $855,674,628,608$ and 610 to $567 \mathrm{~mA} \mathrm{~h} \mathrm{~g}^{-1}$. When the current density returned to $0.2 \mathrm{C}$, a high capacity of $1039 \mathrm{~mA} \mathrm{~h} \mathrm{~g}^{-1}$ was retained even after 100 cycles (Figure 12d). Clearly, the rate performance of $\mathrm{Co}-\mathrm{P} /$ graphene was better than their $\mathrm{Co}_{2} \mathrm{P}-\mathrm{Co}$ counterparts, attributed to the presence of graphene, which improved the electrical conductivity of the electrode (Figure 12e).

Yang and co-workers synthesized a composite with cobalt phosphide nanowires and reduced the graphene oxide (CoP/RGO) [135]. As shown in Figure 12f, the cycle performances of CoP and $\mathrm{CoP} / \mathrm{RGO}$ as anode materials of LIBs were displayed. The CoP/RGO electrode exhibited a higher capacity of $967 \mathrm{~mA} \mathrm{~h} \mathrm{~g}^{-1}$ after 200 cycles. With regard to the CoP electrode, a capacity of only $429 \mathrm{~mA}$ $\mathrm{h} \mathrm{g}^{-1}$ was retained after 100 cycles. This situation can be ascribed to the framework of CoP/RGO, which possesses more free space in favor of the volume change during cycling. Zhang's group synthesized a carbon-coated $\mathrm{CoP}_{3}$ nanocomposite and tested it as an anode material for SIBs [80]. The cycling performances of $\mathrm{CoP}_{3} @ \mathrm{C}$ and $\mathrm{CoP}_{3}$ at $100 \mathrm{~mA} \mathrm{~g}^{-1}$ are displayed in Figure 12g. The $\mathrm{CoP}_{3} @ \mathrm{C}$ 
electrode exhibited a higher capacity than $\mathrm{CoP}_{3}$ after 80 cycles, suggesting that the presence of the carbon layer contributed to the electrochemical performance of the electrode. Furthermore, Zhu's group successfully embedded ultrafine CoP nanoparticles into the carbon nanorod, which delivered a superior cycling performance when tested as an anode material of LIBs at $1 \mathrm{Ag}^{-1}$ (Figure 12h) [142]. Well-utilized $\mathrm{CoP}$ was the main reason for this result.

\subsection{Cobalt Selenide and Its Composites}

Cobalt selenide, one of the transition metal chalcogenides, has attracted enormous attention in the field of anode materials for LIBs or SIBs owing to its good electrical conductivity and high theoretical capacity. Nevertheless, its poor structure stability during cycling is the main drawback of cobalt selenide $[20,37,71,168-170,190-195]$. As an anode material of LIBs, the $\mathrm{Co}_{0.85} \mathrm{Se}$ NSs/G composite synthesized by Zhou's group delivered a high specific capacity of $730 \mathrm{~mA} \mathrm{~h} \mathrm{~g}{ }^{-1}$ after 300 cycles, as shown in Figure 13a [160]. With regard to $\mathrm{Co}_{0.85} \mathrm{Se}$ NSs and $\mathrm{Co}_{0.85} \mathrm{Se}$ NSs without a binder, the specific capabilities rapidly decreased to $50 \mathrm{~mA} \mathrm{~h} \mathrm{~g}^{-1}$ after 50 cycles at $0.5 \mathrm{~A} \mathrm{~g}^{-1}$. It should be noticed that the capacity of $\mathrm{Co}_{0.85} \mathrm{Se} \mathrm{NSs} / \mathrm{G}$ has a slight increase during cycling, ascribed to the presence of graphene and the formation of a polymetric gel-like film. Moreover, the Na-ion storage cyclic performances of $\mathrm{Co}_{0.85} \mathrm{Se} \mathrm{NSs} / \mathrm{G}, \mathrm{Co}_{0.85}$ Se NSs and $\mathrm{Co}_{0.85}$ Se NSs without additives are displayed in Figure 13b [160]. Similar to lithium storage performances, the $\mathrm{Co}_{0.85} \mathrm{Se} \mathrm{NSs} / \mathrm{G}$ electrode exhibited a higher reversible capacity of $193.8 \mathrm{~mA} \mathrm{~h} \mathrm{~g}^{-1}$ after 100 cycles. The better lithium/sodium storage performance of the $\mathrm{Co}_{0.85} \mathrm{Se} \mathrm{NSs} / \mathrm{G}$ composite can be attributed to its unique morphology and composition.

Pan's group successfully fabricated a composite with CoSe nanoparticles uniformly distributed in porous carbon polyhedral (CoSe@PCP) and tested as an anode material for LIBs and SIBs [68]. In Figure 13c, the CoSe@PCP electrode exhibited reversible capabilities of 701.2, 645.6, 590 and $457.5 \mathrm{~mA} \mathrm{~h} \mathrm{~g}^{-1}$ when tested at $0.1,0.5,1$ and $2 \mathrm{~A} \mathrm{~g}^{-1}$, respectively. Moreover, the capacity could recover to $441.8,452.7$ and $524.1 \mathrm{~mA} \mathrm{~h} \mathrm{~g}^{-1}$ when the current density was back to $1,0.5$ and $0.1 \mathrm{~A} \mathrm{~g}^{-1}$, respectively. When utilized as an anode material for SIBs, the electrode delivered the capacities of 360.3, $315.6,278.9,247.1$ and $207.7 \mathrm{~mA} \mathrm{~h} \mathrm{~g}^{-1}$ tested at $0.05,0.25,1,2$ and $4 \mathrm{~A} \mathrm{~g}^{-1}$, respectively. Furthermore, the capacities could recover to the original levels when the current densities returned to $0.5,0.25$ and $0.05 \mathrm{~A} \mathrm{~g}^{-1}$ (Figure 13d). Clearly, the CoSe@PCP electrode shows excellent rate performances when applied as anode materials for LIBs and SIBs, attributed to the novel structure of CoSe@PCP.

Chen's group synthesized a novel urchin-like $\mathrm{CoSe}_{2}$ and firstly used it as an anode material for SIBs [190]. As shown in Figure 13e, the $\mathrm{CoSe}_{2}$ electrode delivered a high capacity of $0.410 \mathrm{~A} \mathrm{~h} \mathrm{~g}^{-1}$ after 1800 cycles at $1 \mathrm{~A} \mathrm{~g}^{-1}$, suggesting a superior cyclic performance. This result can be ascribed to the unique structure of the sample. 

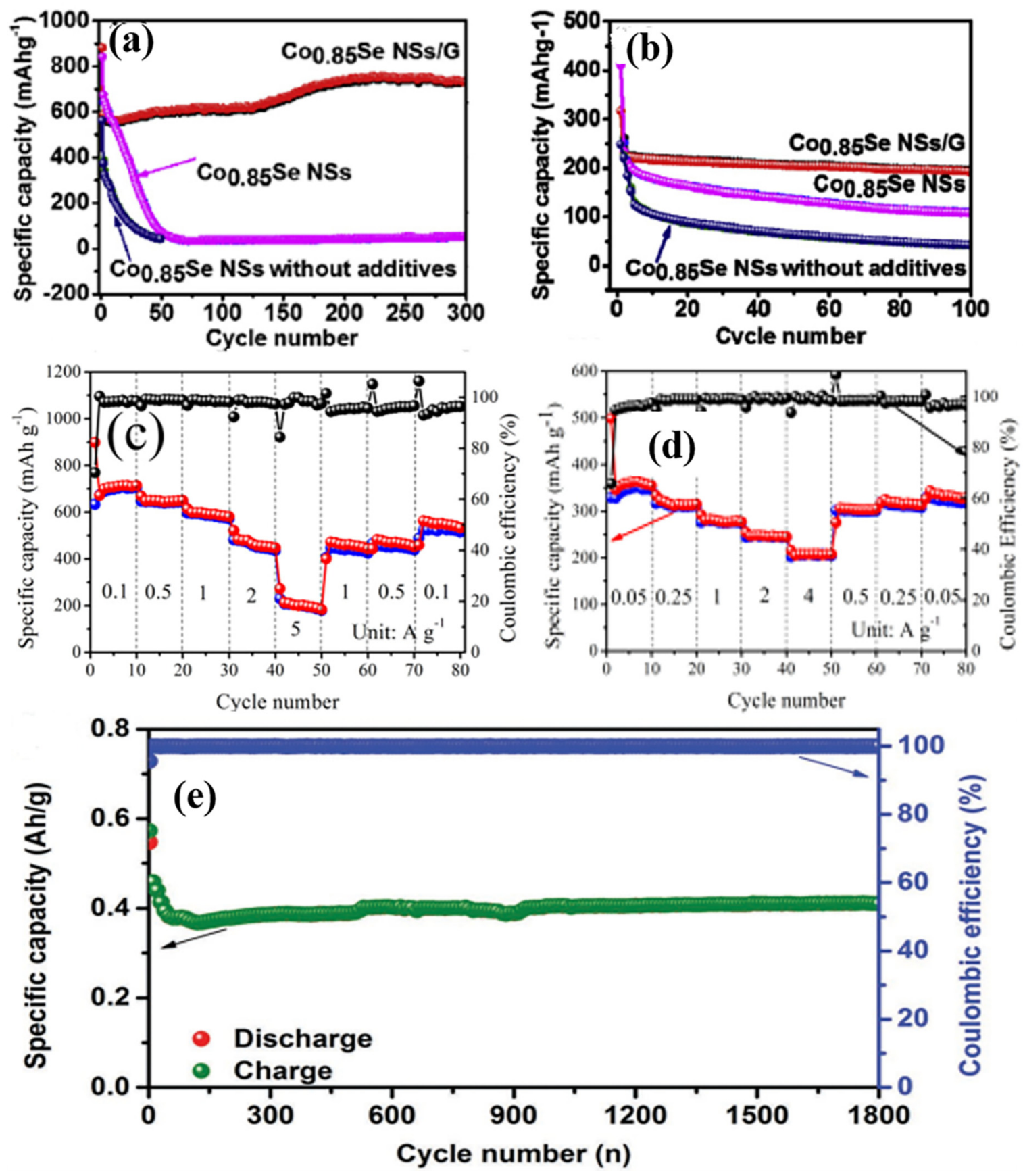

Figure 13. Electrochemical performances of cobalt-based anode materials. (a) Li-ion storage and (b) Na-ion storage cyclic performance of the $\mathrm{Co}_{0.85}$ Se nanosheets/graphene (NSs/G) at $0.5 \mathrm{~A} \mathrm{~g}^{-1}$, in comparison with $\mathrm{Co}_{0.85}$ Se NSs and $\mathrm{Co}_{0.85}$ Se NSs without additives [160]. Rate performance of CoSe@PCP for (c) LIBs and (d) SIBs [68]. (e) Cyclic properties of CoSe 2 at $1 \mathrm{~A} \mathrm{~g}^{-1}$ [190].

\subsection{Other Cobalt-Based Anode Materials}

In addition to cobalt alloys, cobalt oxides, cobalt phosphide and cobalt chalcogenides, other cobalt-based anode materials like $\mathrm{CoMn}_{2} \mathrm{O}_{4}$ have also been utilized in LIBs and SIBs $[39,50-52,72,96,108,109]$. Zhang's group synthesized $\mathrm{CoMn}_{2} \mathrm{O}_{4}$ microspheres and evaluated them as anode materials for LIBs [51]. As displayed in Figure 14a, the $\mathrm{CoMn}_{2} \mathrm{O}_{4}$ electrode exhibited high initial charge and discharge capacities of 1860 and $2296 \mathrm{~mA} \mathrm{~h} \mathrm{~g}^{-1}$ at $100 \mathrm{~mA} \mathrm{~g}^{-1}$, respectively. During the first 200 cycles, the capacity gradually declined owing to a severe structure degradation caused by the conversion reaction. 

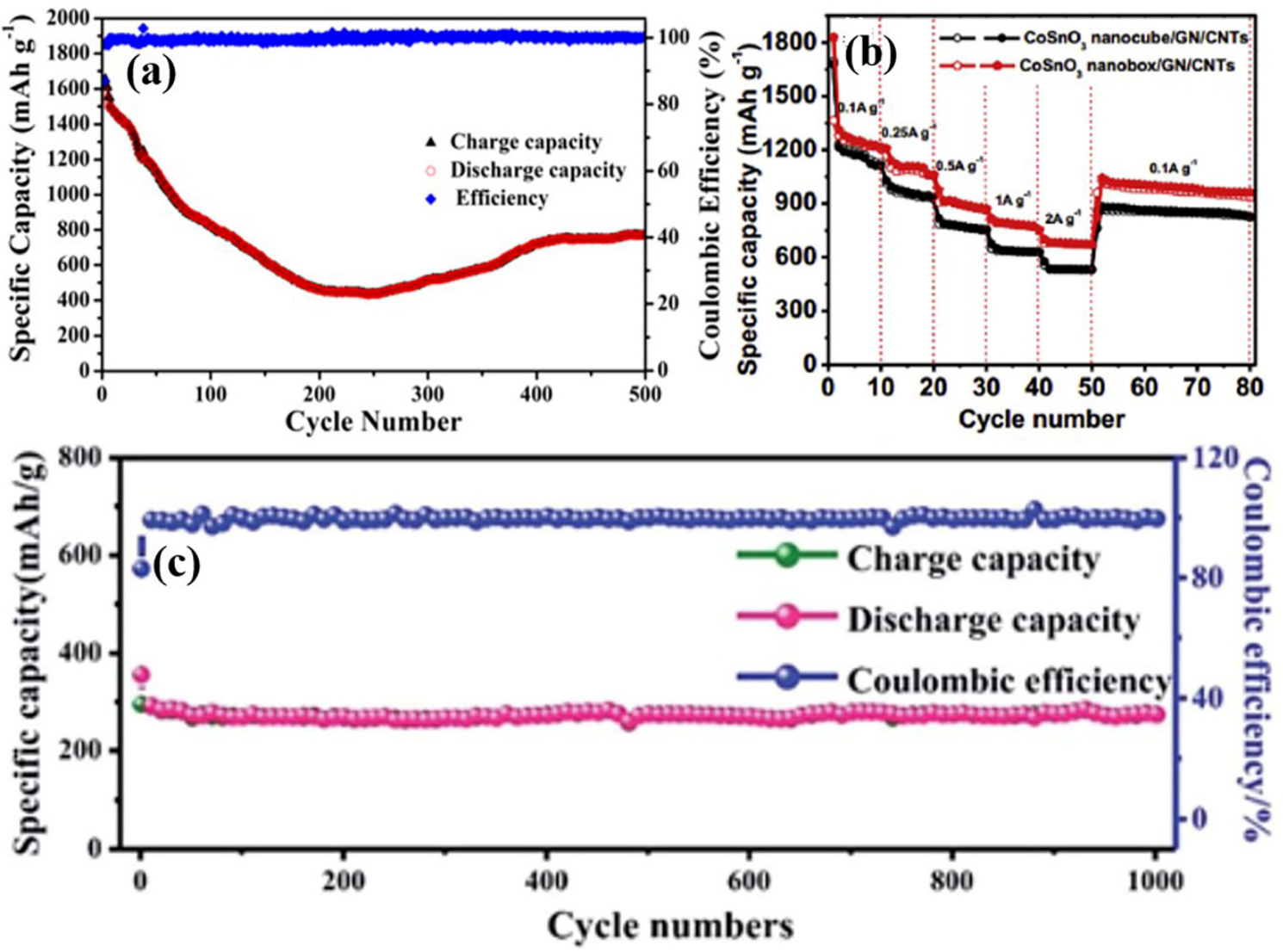

Figure 14. Electrochemical performances of cobalt-based anode materials. (a) Cyclic performance of $\mathrm{CoMn}_{2} \mathrm{O}_{4}$ microspheres at $100 \mathrm{~mA} \mathrm{~g}^{-1}$ in LIBs [51]. (b) Rate performance of $\mathrm{CoSnO}_{3}$ nanobox/graphene (GN)/carbon nanotubes (CNTs) and $\mathrm{CoSnO}_{3}$ nanocube/GN/CNTs as anode materials of LIBs [109]. (c) Cyclic properties of $\mathrm{CoSnO}_{3}-\mathrm{N}$ doped $\mathrm{C}$ (NC)-120 at $1 \mathrm{~A} \mathrm{~g}^{-1}$ [52].

After 250 cycles, the reversible capacities increased, which can be ascribed to the gradual formation of the SEI and the degradation of the electrolyte. After 500 cycles, the $\mathrm{CoMn}_{2} \mathrm{O}_{4}$ electrode delivered a capacity of $722 \mathrm{~mA} \mathrm{~h} \mathrm{~g}$, indicating the enhanced performance of the $\mathrm{CoMn}_{2} \mathrm{O}_{4}$ electrode as an anode for LIBs. Wang et al. prepared $\mathrm{CoSnO}_{3} / \mathrm{GN} / \mathrm{CNTs}$ composite papers that delivered excellent electrochemical properties [109]. The rate performances of the $\mathrm{CoSnO}_{3} / \mathrm{GN} / \mathrm{CNTs}$ composite with different structures are displayed in Figure 14b. The $\mathrm{CoSnO}_{3}$ nanobox/GN/CNTs composite delivered the capacities of $1233.8,1106.4,894.7,780.3$ and $676.7 \mathrm{~mA} \mathrm{~h} \mathrm{~g}^{-1}$ at the current densities of $0.1,0.25,0.5$, 1 and $2 \mathrm{~A} \mathrm{~g}^{-1}$, respectively. Moreover, a high reversible capability of $992.8 \mathrm{~mA} \mathrm{~h} \mathrm{~g}^{-1}$ could be retained when the current density returned to $0.1 \mathrm{~A} \mathrm{~g}^{-1}$. The rate properties of $\mathrm{CoSnO}_{3}$ nanocube/GN/CNTs were poorer than $\mathrm{CoSnO}_{3}$ nanobox/GN/CNTs. Ji's group synthesized $\mathrm{CoSnO}_{3}$-NCs nanoboxes and employed them as anode materials for SIBs [52]. The cyclic performance of this sample was tested at a current density of $1 \mathrm{~A} \mathrm{~g}^{-1}$, as shown in Figure 14c. The $\mathrm{CoSnO}_{3}-\mathrm{NC}$ electrode exhibited a high capacity of $273.8 \mathrm{~mA} \mathrm{~h} \mathrm{~g}^{-1}$ after 1000 cycles owing to the stable structure derived from the presence of the carbon layer.

The electrochemical properties of typical cobalt-based anode materials for LIBs and SIBs are summarized in Table 3. Clearly, this cobalt-based active material delivered different levels of rate performances and cyclic stabilities owing to the differences between morphology and composition. Hence, a rational structure is vitally important for the electrochemical properties of cobalt-based anode materials. Furthermore, the combination with carbonaceous materials is also an effective method to enhance the electrochemical properties of cobalt-based anode materials for LIBs and SIBs. 
Table 3. Summary of the Li/Na-storage performances of typical cobalt-based anode materials. LIBs: lithium ion batteries and SIBs: sodium ion batteries.

\begin{tabular}{|c|c|c|c|c|c|}
\hline Types of Materials & $\begin{array}{l}\text { Current Density } \\
\left(\mathrm{A} \mathrm{g}^{-1}\right)\end{array}$ & $\begin{array}{c}\text { Cut-off } \\
\text { Voltage (V) }\end{array}$ & $\begin{array}{c}\text { Cycle } \\
\text { Number }\end{array}$ & $\begin{array}{c}\text { Specific Capacity } \\
\left(\mathrm{mA} \mathrm{h} \mathrm{g}^{-1}\right)\end{array}$ & Reference \\
\hline \multicolumn{6}{|l|}{ For LIBs } \\
\hline Sn-Co@C-2 & 0.1 & $0.01-3$ & 100 & 818 & [64] \\
\hline SnCo@CNT-3DC & 0.1 & $0.005-3$ & 100 & 826 & [44] \\
\hline Sn-Co@PMMA & 0.1 & $0.001-2$ & 100 & 594 & [40] \\
\hline $\mathrm{Co}_{3} \mathrm{Sn}_{2} @ \mathrm{Co}-\mathrm{NG}$ & 0.25 & $0.005-3$ & 100 & 1615 & [53] \\
\hline SnCo/PAN-CNFs & 0.267 & $0.005-2.5$ & 100 & 548 & [92] \\
\hline Co-Sn/CNF-800 & 0.161 & $0.02-2.8$ & 80 & 560 & [90] \\
\hline Sn-Co-CNT@CNT & 0.099 & $0.005-3$ & 200 & 811 & [67] \\
\hline GNS-SnCo & 0.072 & $0.005-3$ & 60 & 571 & [106] \\
\hline $\mathrm{Sb}-\mathrm{Co}-\mathrm{P}$ & 0.1 & $0.02-1.5$ & 50 & 539 & {$[50]$} \\
\hline CoSnC & 0.1 & $0-2$ & 50 & 450 & [108] \\
\hline $\mathrm{Co}_{2} \mathrm{SnO}_{4}$ & 0.1 & $0.01-2.5$ & 100 & 1000 & [39] \\
\hline $\mathrm{CoSnO}_{3} / \mathrm{GN} / \mathrm{CNTs}$ & 0.1 & $0-3$ & 150 & 1098.7 & [109] \\
\hline $\mathrm{CoMn}_{2} \mathrm{O}_{4}$ & 0.1 & $0.001-3$ & 500 & 772 & [51] \\
\hline $\mathrm{CoO}_{\mathrm{x}} / \mathrm{MCS}$ & 0.07 & $0.01-3$ & 30 & 703 & [100] \\
\hline $\mathrm{Co}_{3} \mathrm{O}_{4} /$ Graphene & 0.2 & $0.001-3$ & 42 & 800 & [110] \\
\hline $\mathrm{Co}_{3} \mathrm{O}_{4}$ nanobelts & 1 & $0-3$ & 60 & 614 & [57] \\
\hline $\mathrm{Co}_{3} \mathrm{O}_{4}$ nanorods/GNS & 1 & $0.01-3$ & 40 & 1090 & [113] \\
\hline Shale-like $\mathrm{Co}_{3} \mathrm{O}_{4}$ & 0.2 & $0.005-2.9$ & 100 & 1045.3 & {$[35]$} \\
\hline $\mathrm{Co}_{3} \mathrm{O}_{4}$ nanoflakes & 0.089 & $0.01-3$ & 300 & 806 & [118] \\
\hline $\begin{array}{l}\text { Peapod-like } \\
\mathrm{Co}_{3} \mathrm{O}_{4} @ \mathrm{CNT}\end{array}$ & 0.1 & $0-3$ & 60 & 318 & [121] \\
\hline $\mathrm{CoP} / \mathrm{C}$ & 0.1 & $0-2$ & 200 & 407 & [134] \\
\hline CoP microflake & 1 & $0.01-3$ & 800 & 619.2 & [136] \\
\hline $\mathrm{CoP} / \mathrm{Co}_{2} \mathrm{P}$ & 0.2 & $0.01-3$ & 450 & 851.2 & [137] \\
\hline CoP nanorods & 0.5 & $0.01-3$ & 300 & 894 & [138] \\
\hline $\mathrm{Co}_{2} \mathrm{P}-\mathrm{Co}$ & 0.1 & $0.01-3$ & 200 & 929 & [139] \\
\hline CoP nanorod arrays & 0.4 & $0.01-3$ & 900 & 390 & [61] \\
\hline CoP HR@rGO & 0.1 & $0.005-3$ & 100 & 714.7 & {$[25]$} \\
\hline CoP@C/BC & 1 & $0.01-3$ & 1000 & 351 & [142] \\
\hline $\mathrm{CoP}_{3} @ \mathrm{PPy}$ & 0.5 & $0-2$ & 220 & 650 & [144] \\
\hline $\mathrm{CoS}_{2} \mathrm{NP} @ \mathrm{G}-\mathrm{CoS}_{2} \mathrm{QD}$ & 1 & $0.05-3$ & 300 & 831 & [187] \\
\hline Worm-like $\mathrm{CoS}_{2}$ & 0.1 & $0.01-3$ & 100 & 883 & [180] \\
\hline $\mathrm{CoS}_{2} @ \mathrm{NG}$ & 0.1 & $0.01-3$ & 150 & 882 & {$[26]$} \\
\hline $\mathrm{NC} / \mathrm{CoS}_{2}-650$ & 0.1 & $0.1-3$ & 50 & 560 & {$[66]$} \\
\hline Mesoporous $\mathrm{Co}_{9} \mathrm{~S}_{8}$ & 2 & $0.01-3$ & 800 & 896 & [175] \\
\hline MWCNT@a-C@Cog $S_{8}$ & 2 & $0.01-3$ & 700 & 1065 & {$[5]$} \\
\hline $\mathrm{CoS}_{2}$ nanobubble & 1 & $0.05-3$ & 200 & 737 & [174] \\
\hline $\mathrm{rGO} / \mathrm{CoSe}_{2}$ & 0.2 & $0.01-3$ & 200 & 1577 & [20] \\
\hline $\mathrm{Co}_{0.85}$ Se nanosheets & 0.2 & $0.01-3$ & 50 & 516 & [194] \\
\hline $\mathrm{Cu}$-doped $\mathrm{CoSe}_{2}$ & 1 & $0.01-3$ & 200 & 807 & [195] \\
\hline CoSe@PCP & 1 & $0.005-3$ & 500 & 708.2 & {$[68]$} \\
\hline $\mathrm{CoSe}_{2} @ \mathrm{C} / \mathrm{CNTs}$ & 1 & $0.5-2.9$ & 1000 & 390 & [48] \\
\hline $\mathrm{CoSe}_{2} @ \mathrm{CNFs}$ & 0.2 & $0.01-3$ & 300 & 1405 & [93] \\
\hline \multicolumn{6}{|l|}{ For SIBs } \\
\hline SnCo@C & 0.1 & $0.1-2$ & 120 & 276.2 & [41] \\
\hline CoSnO3-NCs & 1 & $0.01-3$ & 1000 & 273.8 & [52] \\
\hline Shale-like $\mathrm{Co}_{3} \mathrm{O}_{4}$ & 0.05 & $0.005-2.9$ & 50 & 380 & {$[35]$} \\
\hline $\mathrm{Co}_{3} \mathrm{O}_{4} @ \mathrm{CNT}$ & 0.05 & $0.01-3$ & 100 & 403 & [122] \\
\hline $\mathrm{Co}_{3} \mathrm{O}_{4} @ \mathrm{NC}$ & 1 & $0.01-3$ & 1100 & 175 & {$[124]$} \\
\hline $\mathrm{Ni}$-doped $\mathrm{Co} / \mathrm{CoO} / \mathrm{NC}$ & 0.5 & $0.01-3$ & 100 & 218.7 & {$[126]$} \\
\hline Yolk-shell $\mathrm{Co}_{3} \mathrm{O}_{4} / \mathrm{C}$ & 1 & $0.01-3$ & 200 & 240 & [129] \\
\hline $\mathrm{Co}_{3} \mathrm{O}_{4} / \mathrm{MnO}_{2} @ \mathrm{C}$ & 0.8 & $0.01-3$ & 1000 & 126 & [91] \\
\hline CoP@C-RGO-NF & 0.05 & $0.01-3$ & 100 & 473.1 & [27] \\
\hline RGO@CoP@C-FeP & 0.1 & $0.01-3$ & 200 & 456.2 & {$[70]$} \\
\hline $\mathrm{A}-\mathrm{Co}_{2} \mathrm{P} / \mathrm{C}_{\mathrm{x}} \mathrm{N}_{\mathrm{y}} \mathrm{B}_{2}-650$ & 0.2 & $0.005-2.5$ & 100 & 251.2 & [140] \\
\hline CoP nanorod arrays & 0.2 & $0.01-3$ & 550 & 297 & [61] \\
\hline $\mathrm{CoP}-\mathrm{O}$ & 1 & $0.01-2.5$ & 900 & 386 & {$[13]$} \\
\hline $\mathrm{Co}_{2} \mathrm{P} @ \mathrm{~N}-\mathrm{C} @ \mathrm{rGO}$ & 0.05 & $0.01-3$ & 100 & 225 & [143] \\
\hline $\mathrm{CoP}_{3} @ \mathrm{C}$ & 0.1 & $0-2.5$ & 80 & 212 & {$[80]$} \\
\hline
\end{tabular}


Table 3. Cont.

\begin{tabular}{|c|c|c|c|c|c|}
\hline Types of Materials & $\begin{array}{l}\text { Current Density } \\
\left(\mathrm{A} \mathrm{g}^{-1}\right)\end{array}$ & $\begin{array}{c}\text { Cut-off } \\
\text { Voltage (V) }\end{array}$ & $\begin{array}{c}\text { Cycle } \\
\text { Number }\end{array}$ & $\begin{array}{l}\text { Specific Capacity } \\
\left(\mathrm{mA} \mathrm{h} \mathrm{g}^{-1}\right)\end{array}$ & Reference \\
\hline $\mathrm{CoS}_{2}-\mathrm{MWCNT}$ & 0.1 & $1-2.9$ & 100 & 568 & [47] \\
\hline $\mathrm{cs}-\mathrm{Co}_{\mathrm{x}} \mathrm{S}_{\mathrm{y}} / \mathrm{DPC}$ & 0.5 & $0.01-3$ & 50 & 300 & [181] \\
\hline $\mathrm{CoS}_{2} / \mathrm{rGO}$ & 1 & $0.01-3$ & 1000 & 192 & [49] \\
\hline CoS@rGO & 1 & $0.1-2.9$ & 1000 & 420 & [173] \\
\hline$\left(\mathrm{Co}_{9} \mathrm{~S}_{8} \mathrm{QD} @ \mathrm{HCP}\right) @ \mathrm{rGO}$ & 0.3 & $0.01-2.9$ & 500 & 628 & [172] \\
\hline CoS@rGO & 1 & $0.01-2.9$ & 1000 & 420 & [173] \\
\hline $\mathrm{CoS}_{\mathrm{x}} @ \mathrm{NSC}$ & 1 & $0.01-3$ & 200 & 606 & [186] \\
\hline $\mathrm{Co}_{9} \mathrm{~S}_{8} @ \mathrm{C}$ & 5 & $0.01-3$ & 1000 & 305 & [23] \\
\hline Urchin-like $\mathrm{CoSe}_{2}$ & 1 & $0.5-3$ & 1800 & 410 & [190] \\
\hline CoSe@PCP & 0.1 & $0.005-3$ & 100 & 341 & [68] \\
\hline $\mathrm{CoSe}_{2}$ nanorods & 5 & $0.4-3$ & 2000 & 386 & [168] \\
\hline $\mathrm{CoSe}_{2} @ \mathrm{~N}-\mathrm{PGC} / \mathrm{CNTs}$ & 0.2 & $0.001-3$ & 100 & 424 & {$[48]$} \\
\hline $\mathrm{Co}_{9} \mathrm{Se}_{8} / \mathrm{rGO}$ & 0.05 & $0.01-3$ & 100 & 406 & [97] \\
\hline CoSe/C & 0.5 & $0.01-3$ & 50 & 552.5 & [166] \\
\hline CoSe@100CSs & 4 & $0.5-2.8$ & 10,000 & 260 & {$[60]$} \\
\hline $\mathrm{CoSe}_{2} / \mathrm{N}-\mathrm{CNF}$ & 2 & $0.5-3$ & 1000 & 308.4 & [24] \\
\hline
\end{tabular}

\section{Conclusions and Perspectives}

The increasing consumption of fossil energy makes the development of clean energy necessary. Due to time and geographical constraints, high-performance RBs are urgent for the development of clean energy. Designing and synthesizing suitable electrode materials is critical for the development of RBs. This review systematically demonstrates that cobalt-based electrode materials are expected to be a new generation of anode materials for LIBs/SIBs.

In this review, we sequentially summarized the $\mathrm{Li}^{+} / \mathrm{Na}^{+}$storage mechanism, typical preparation methods and applications of various cobalt-based anode materials, including cobalt-based alloy, cobalt oxide, cobalt sulfides and cobalt phosphides. For cobalt-based anode materials, their large volume changes and poor conductivity during charges and discharges are the main obstacles to the further development in LIBs/SIBs. In this regard, future research can focus on the following aspects:

(1) Developing a simple and feasible synthetic process for the preparation of cobalt-based anode materials with specific morphology and sizes, which can ensure satisfactory contact between the electrolyte and active material, and cycle performances of cobalt-based anode materials. In addition, the combination with the conductive material can improve the conductivity of the electrode material and improve the rate performances of the cobalt-based anode materials.

(2) More advanced characterization and calculation methods should be used to further study the $\mathrm{Li}^{+} / \mathrm{Na}^{+}$storage mechanisms of cobalt-based anode materials, which will be meaningful for designing a suitable morphology.

(3) Considering commercial applications, the whole battery system, including cathode materials, binders, conductive agents, electrolytes and additives, should also be optimized. Appropriate matching materials can fully exploit the advantages of the high storage capacities of cobalt-based anode materials in LIBs/SIBs.

Author Contributions: Y.Z. and N.W. wrote the manuscript. Z.B. revised the manuscript. All authors commented on the manuscript and have contributed equally to this work. All authors have read and agreed to the published version of the manuscript.

Funding: This work was supported by the Australian Research Council (ARC; DE200101384) for the support provided through a Discovery Project (DP160102627) and a Linkage Project (LP160100273) and the Shanxi Province Science Foundation for Youths (No. 201901D211257).

Acknowledgments: We acknowledge the support from Australian Research Council for the support provided through a Discovery Project and a Linkage Project and the Shanxi Province Science Foundation for Youths.

Conflicts of Interest: The authors declare no conflict of interest. 


\section{References}

1. Xue, P.; Wang, N.; Wang, Y.; Zhang, Y.; Liu, Y.; Tang, B.; Bai, Z.; Dou, S. Nanoconfined SnS in 3D interconnected macroporous carbon as durable anodes for lithium/sodium ion batteries. Carbon 2018, 134, 222-231. [CrossRef]

2. Lu, Z.; Wang, N.; Zhang, Y.; Xue, P.; Guo, M.; Tang, B.; Bai, Z.; Dou, S. Pyrite FeS ${ }_{2} @$ nanorods as smart cathode for sodium ion battery with ultra-long lifespan and notable rate performance from tunable pseudocapacitance. Electrochim. Acta 2018, 260, 755-761. [CrossRef]

3. Bai, Z.; Fan, N.; Ju, Z.; Guo, C.; Qian, Y.; Tang, B.; Xiong, S. Facile synthesis of mesoporous $\mathrm{Mn}_{3} \mathrm{O}_{4}$ nanotubes and their excellent performance for lithium-ion batteries. J. Mater. Chem. A 2013, 1, 10985. [CrossRef]

4. Bai, Z.; Ju, Z.; Guo, C.; Qian, Y.; Tang, B.; Xiong, S. Direct large-scale synthesis of 3D hierarchical mesoporous $\mathrm{NiO}$ microspheres as high-performance anode materials for lithium ion batteries. Nanoscale 2014, 6, 3268-3273. [CrossRef] [PubMed]

5. Zhou, Y.; Yan, D.; Xu, H.; Liu, S.; Yang, J.; Qian, Y. Multiwalled carbon nanotube@a-C@Co $\mathrm{S}_{8}$ nanocomposites: A high-capacity and long-life anode material for advanced lithium ion batteries. Nanoscale 2015, 7, 3520-3525. [CrossRef] [PubMed]

6. Bai, Z.; Zhang, Y.; Zhang, Y.; Guo, C.; Tang, B. Hierarchical MoS $2 @$ Carbon Microspheres as Advanced Anodes for Li-Ion Batteries. Chem-Eur. J. 2015, 21, 18187-18191. [CrossRef]

7. Bai, Z.; Zhang, Y.; Zhang, Y.; Guo, C.; Tang, B.; Sun, D. MOFs-derived porous $\mathrm{Mn}_{2} \mathrm{O}_{3}$ as high-performance anode material for Li-ion battery. J. Mater. Chem. A 2015, 3, 5266-5269. [CrossRef]

8. Wang, N.; Xu, Z.; Xu, X.; Liao, T.; Tang, B.; Bai, Z.; Dou, S. Synergistically Enhanced Interfacial Interaction to Polysulfide via N,O Dual-Doped Highly Porous Carbon Microrods for Advanced Lithium-Sulfur Batteries. ACS Appl. Mater. Interfaces 2018, 10, 13573-13580. [CrossRef]

9. Wang, N.; Wang, Y.; Xu, X.; Liao, T.; Du, Y.; Bai, Z.; Dou, S. Defect Sites-Rich Porous Carbon with Pseudocapacitive Behaviors as an Ultrafast and Long-Term Cycling Anode for Sodium-Ion Batteries. ACS Appl. Mater. Interfaces 2018, 10, 9353-9361. [CrossRef]

10. Zhang, Y.; Zhang, Y.; Guo, C.; Tang, B.; Wang, X.; Bai, Z. Porous $\mathrm{ZnMn}_{2} \mathrm{O}_{4}$ nanowires as an advanced anode material for lithium ion battery. Electrochim. Acta 2015, 182, 1140-1144. [CrossRef]

11. Zhang, C.-L.; Lu, B.-R.; Cao, F.-H.; Yu, Z.-L.; Cong, H.P.; Yu, S.H. Hierarchically structured $\mathrm{Co}_{3} \mathrm{O}_{4} @{ }_{\text {carbon }}$ porous fibers derived from electrospun ZIF-67/PAN nanofibers as anodes for lithium ion batteries. J. Mater. Chem. A 2018, 6, 12962-12968. [CrossRef]

12. Zhang, Y.; Wang, N.; Sun, C.; Lu, Z.; Xue, P.; Tang, B.; Bai, Z.; Dou, S. 3D spongy CoS $_{2}$ nanoparticles/carbon composite as high-performance anode material for lithium/sodium ion batteries. Chem. Eng. J. 2018, 332, 370-376. [CrossRef]

13. Zhang, K.; Park, M.; Zhang, J.; Lee, G.-H.; Shin, J.; Kang, Y.-M. Cobalt phosphide nanoparticles embedded in nitrogen-doped carbon nanosheets: Promising anode material with high rate capability and long cycle life for sodium-ion batteries. Nano Res. 2017, 10, 4337-4350. [CrossRef]

14. Wang, Q.; Jiao, L.; Han, Y.; Du, H.; Peng, W.; Huan, Q.; Song, D.; Si, Y.; Wang, Y.; Yuan, H. CoS 2 Hollow Spheres: Fabrication and Their Application in Lithium-Ion Batteries. J. Phy. Chem. C 2011, 115, 8300-8304. [CrossRef]

15. Kang, Y.-M.; Song, M.-S.; Kim, J.-H.; Kim, H.-S.; Park, M.-S.; Lee, J.-Y.; Liu, H.K.; Dou, S.X. A study on the charge-discharge mechanism of $\mathrm{Co} 3 \mathrm{O} 4$ as an anode for the Li ion secondary battery. Electrochim. Acta 2005, 50, 3667-3673. [CrossRef]

16. Wang, Y.; Wu, J.; Tang, Y.; Lu, X.; Yang, C.; Qin, M.; Huang, F.; Li, X.; Zhang, X. Phase-controlled synthesis of cobalt sulfides for lithium ion batteries. ACS Appl. Mater. Interfaces 2012, 4, 4246-4250. [CrossRef]

17. Gu, Y.; Xu, Y.; Wang, Y. Graphene-wrapped CoS nanoparticles for high-capacity lithium-ion storage. ACS Appl. Mater. Interfaces 2013, 5, 801-806. [CrossRef]

18. DeMattei, R.C.; Watcharapasorn, A.; Feigelson, R.S. Conditions for the Electrochemical Synthesis of the CoPn (Pn=P, As, Sb) Skutterudites. J. Electrochem. Soc. 2001, 148, D109. [CrossRef]

19. Ferguson, P.P.; Todd, A.D.W.; Dahn, J.R. Comparison of mechanically alloyed and sputtered tin-cobalt-carbon as an anode material for lithium-ion batteries. Electrochem. Commun. 2008, 10, 25-31. [CrossRef]

20. Li, Z.; Xue, H.; Wang, J.; Tang, Y.; Lee, C.-S.; Yang, S. Reduced Graphene Oxide/Marcasite-Type Cobalt Selenide Nanocrystals as an Anode for Lithium-Ion Batteries with Excellent Cyclic Performance. ChemElectroChem 2015, 2, 1682-1686. [CrossRef] 
21. Liu, Y.; Mi, C.; Su, L.; Zhang, X. Hydrothermal synthesis of $\mathrm{Co}_{3} \mathrm{O}_{4}$ microspheres as anode material for lithium-ion batteries. Electrochim. Acta 2008, 53, 2507-2513. [CrossRef]

22. Guo, H.; Zhao, H.; Jia, X.; Li, X.; Qiu, W. A novel micro-spherical $\mathrm{CoSn}_{2} / \mathrm{Sn}$ alloy composite as high capacity anode materials for Li-ion rechargeable batteries. Electrochim. Acta 2007, 52, 4853-4857. [CrossRef]

23. Zhang, Y.; Wang, N.; Xue, P.; Liu, Y.; Tang, B.; Bai, Z.; Dou, S. Cog $\mathrm{S}_{8} @$ carbon nanospheres as high-performance anodes for sodium ion battery. Chem. Eng. J. 2018, 343, 512-519. [CrossRef]

24. Cui, C.; Wei, Z.; Zhou, G.; Wei, W.; Ma, J.; Chen, L.; Li, C. Quasi-reversible conversion reaction of $\mathrm{CoSe}_{2} /$ nitrogen-doped carbon nanofibers towards long-lifetime anode materials for sodium-ion batteries. $J$. Mater. Chem. A 2018, 6, 7088-7098. [CrossRef]

25. Han, Z.; Wang, B.; Liu, X.; Wang, G.; Wang, H.; Bai, J. Peapod-like one-dimensional (1D) CoP hollow nanorods embedded into graphene networks as an anode material for lithium-ion batteries. J. Mater. Sci. 2018, 53, 8445-8459. [CrossRef]

26. Qiu, W.; Jiao, J.; Xia, J.; Zhong, H.; Chen, L. A self-standing and flexible electrode of yolk-shell $\mathrm{CoS}_{2}$ spheres encapsulated with nitrogen-doped graphene for high-performance lithium-ion batteries. Chem-Eur. J. 2015, 21, 4359-4367. [CrossRef]

27. Ge, X.; Li, Z.; Yin, L. Metal-organic frameworks derived porous core/shellCoP@C polyhedrons anchored on 3D reduced graphene oxide networks as anode for sodium-ion battery. Nano Energy 2017, 32, 117-124. [CrossRef]

28. Zeng, P.; Li, J.; Ye, M.; Zhuo, K.; Fang, Z. In Situ Formation of $\mathrm{Co}_{9} \mathrm{~S}_{8} / \mathrm{N}-\mathrm{C}$ Hollow Nanospheres by Pyrolysis and Sulfurization of ZIF-67 for High-Performance Lithium-Ion Batteries. Chem-Eur. J. 2017, 23, 9517-9524. [CrossRef]

29. Dong, W.; Shen, D.; Yang, S.; Liang, B.; Wang, X.; Liu, Y.; Li, S. First-principles Study of Mechanical and Electronic Properties of Co-Sn Intermetallics for Lithium Ion Battery Anode. Chem. Res. Chin. Univ. 2018, 34, 235-240. [CrossRef]

30. Hu, X.; Jia, J.; Wang, G.; Chen, J.; Zhan, H.; Wen, Z. Reliable and General Route to Inverse Opal Structured Nanohybrids of Carbon-Confined Transition Metal Sulfides Quantum Dots for High-Performance Sodium Storage. Adv. Energy Mater. 2018, 1801452. [CrossRef]

31. Liu, Z.; Yang, S.; Sun, B.; Chang, X.; Zheng, J.; Li, X. A Peapod-like CoP@C Nanostructure from Phosphorization in a Low-Temperature Molten Salt for High-Performance Lithium-Ion Batteries. Angew. Chem. Int. Ed. Engl. 2018, 130, 10344-10348. [CrossRef]

32. Ge, P.; Hou, H.; Li, S.; Huang, L.; Ji, X. Three-Dimensional Hierarchical Framework Assembled by Cobblestone-Like CoSe2@C Nanospheres for Ultrastable Sodium-Ion Storage. ACS Appl. Mater. Interfaces 2018, 10, 14716-14726. [CrossRef] [PubMed]

33. Ma, Y.; Ma, Y.; Bresser, D.; Ji, Y.; Geiger, D.; Kaiser, U.; Streb, C.; Varzi, A.; Passerini, S. Cobalt Disulfide Nanoparticles Embedded in Porous Carbonaceous Micro-Polyhedrons Interlinked by Carbon Nanotubes for Superior Lithium and Sodium Storage. ACS Nano 2018, 12, 7220-7231. [CrossRef] [PubMed]

34. Hassoun, J.; Mulas, G.; Panero, S.; Scrosati, B. Ternary Sn-Co-C Li-ion battery electrode material prepared by high energy ball milling. Electrochem. Commun. 2007, 9, 2075-2081. [CrossRef]

35. Li, H.-H.; Li, Z.-Y.; Wu, X.L.; Zhang, L.-L.; Fan, C.-Y.; Wang, H.-F.; Li., X.Y.; Wang, K.; Sun, H.-Z.; Zhang, J.-P. Shale-like $\mathrm{CO}_{3} \mathrm{O}_{4}$ for high performance lithium/sodium ion batteries. J. Mater. Chem. 2013, 4, 8242-8248.

36. Juan Xiang, T.S. One-Pot Synthesis of Multicomponent (Mo, Co) Metal Sulfide/Carbon Nanoboxes as anode materials for Improving Na-ion Strorage. Chem. Commun. 2013, 53, 10820-10823. [CrossRef]

37. Dong, S.; Wang, S.; Guan, J.; Li, S.; Lan, Z.; Chen, C.; Shang, C.; Zhang, L.; Wang, X.; Gu, L.; et al. Insight into Enhanced Cycling Performance of $\mathrm{Li}_{-} \mathrm{O}_{2}$ Batteries Based on Binary $\mathrm{CoSe}_{2} / \mathrm{CoO}$ Nanocomposite Electrodes. J. Phys. Chem. Lett. 2014, 5, 615-621. [CrossRef]

38. Du, Z.; Zhang, S. Enhanced Electrochemical Performance of Sn-Co Nanoarchitectured Electrode for Lithium Ion Batteries. J. Phys. Chem. C 2011, 115, 23603-23609. [CrossRef]

39. Zhang, J.; Liang, J.; Zhu, Y.; Wei, D.; Fan, L.; Qian, Y. Synthesis of $\mathrm{Co}_{2} \mathrm{SnO}_{4}$ hollow cubes encapsulated in graphene as high capacity anode materials for lithium-ion batteries. J. Mater. Chem. A 2014, 2, 2728. [CrossRef]

40. Yu, X.; Jiang, A.; Yang, H.; Meng, H.; Dou, P.; Ma, D.; Xu, X. Facile synthesis of hollow Sn-Co@PMMA nanospheres as high performance anodes for lithium-ion batteries via galvanic replacement reaction and in situ polymerization. Appl. Surf. Sci. 2015, 347, 624-631. [CrossRef] 
41. Huang, B.; Yang, J.; Li, Y.; Xiao, S.; Chen, Q. Carbon encapsulated Sn-Co alloy: A stabilized tin-based material for sodium storage. Mater. Lett. 2018, 210, 321-324. [CrossRef]

42. Lee, S.-I.; Yoon, S.; Park, C.-M.; Lee, J.-M.; Kim, H.; Im, D.; Doo, S.-G.; Sohn, H.-J. Reaction mechanism and electrochemical characterization of a $\mathrm{Sn}-\mathrm{Co}-\mathrm{C}$ composite anode for Li-ion batteries. Electrochim. Acta 2008, 54, 364-369. [CrossRef]

43. Fan, X.-Y.; Ke, F.-S.; Wei, G.-Z.; Huang, L.; Sun, S.-G. Sn-Co alloy anode using porous Cu as current collector for lithium ion battery. J. Alloy. Compd. 2009, 476, 70-73. [CrossRef]

44. Qin, J.; Liu, D.; Zhang, X.; Zhao, N.; Shi, C.; Liu, E.Z.; He, F.; Ma, L.; Li, Q.; Li, J.; et al. One-step synthesis of SnCo nanoconfined in hierarchical carbon nanostructures for lithium ion battery anode. Nanoscale 2017, 9 , 15856-15864. [CrossRef] [PubMed]

45. Hassoun, J.; Ochal, P.; Panero, S.; Mulas, G.; Bonatto Minella, C.; Scrosati, B. The effect of CoSn/CoSn 2 phase ratio on the electrochemical behaviour of $\mathrm{Sn}_{40} \mathrm{Co}_{40} \mathrm{C}_{20}$ ternary alloy electrodes in lithium cells. J. Power Sources 2008, 180, 568-575. [CrossRef]

46. López, M.C.; Ortiz, G.F.; Tirado, J.L. A Functionalized $\mathrm{Co}_{2} \mathrm{P}$ Negative Electrode for Batteries Demanding High Li-Potential Reaction. J. Electrochem. Soc. 2012, 159, A1253-A1261. [CrossRef]

47. Shadike, Z.; Cao, M.H.; Ding, F.; Sang, L.; Fu, Z.W. Improved electrochemical performance of $\mathrm{CoS}_{2}-\mathrm{MWCNT}$ nanocomposites for sodium-ion batteries. Chem. Commun. 2015, 51, 10486-10489. [CrossRef]

48. Tang, Y.; Zhao, Z.; Hao, X.; Wang, Y.; Liu, Y.; Hou, Y.; Yang, Q.; Wang, X.; Qiu, J. Engineering hollow polyhedrons structured from carbon-coated $\mathrm{CoSe}_{2}$ nanospheres bridged by CNTs with boosted sodium storage performance. J. Mater. Chem. A 2017, 5, 13591-13600. [CrossRef]

49. Li, Z.; Feng, W.; Lin, Y.; Liu, X.; Fei, H. Flaky $\mathrm{CoS}_{2}$ and graphene nanocomposite anode materials for sodium-ion batteries with improved performance. RSC Adv. 2016, 6, 70632-70637. [CrossRef]

50. Zheng, X.-M.; Xiao, Y.; Huang, L.; Ke, F.-S.; He, Y.; Li, J.-T.; Wei, G.-Z.; Sun, S.-G. Fabrication and electrochemical properties of novel ternary $\mathrm{Sb}-\mathrm{Co}-\mathrm{P}$ alloy electrodes for lithium-ion batteries. Electrochem. Commun. 2009, 11, 1803-1806. [CrossRef]

51. Jiu, H.; Ren, N.; Jiang, L.; Zhang, Q.; Gao, Y.; Meng, Y.; Zhang, L. Hierarchical porous $\mathrm{CoMn}_{2} \mathrm{O}_{4}$ microspheres with sub-nanoparticles as advanced anode for high-performance lithium-ion batteries. J. Solid State Electr. 2018, 22, 2747-2755. [CrossRef]

52. Zou, G.; Hou, H.; Zhao, G.; Ge, P.; Yin, D.; Ji, X. N-rich carbon coated $\mathrm{CoSnO}_{3}$ derived from in situ construction of a Co-MOF with enhanced sodium storage performance. J. Mater. Chem. A 2018, 6, 4839-4847. [CrossRef]

53. Nasir Mahmood, C.Z.; Fei, L.; Jinghan, Z.; Yanglong, H. Hybrid of $\mathrm{Co}_{3} \mathrm{Sn}_{2} @ \mathrm{Co}$ Nanoparticles and Nitrogen-Doped Graphene as a Lithium Ion Battery Anode. ACS Nano 2013, 7, 10307-10318. [CrossRef] [PubMed]

54. Zhan, F.; Geng, B.; Guo, Y. Porous $\mathrm{Co}_{3} \mathrm{O}_{4}$ nanosheets with extraordinarily high discharge capacity for lithium batteries. Chem-Eur. J. 2009, 15, 6169-6174. [CrossRef] [PubMed]

55. He, J.; Zhao, H.; Wang, J.; Wang, J.; Chen, J. Hydrothermal synthesis and electrochemical properties of nano-sized Co-Sn alloy anodes for lithium ion batteries. J. Alloy. Compd. 2010, 508, 629-635. [CrossRef]

56. Wang, X.L.; Han, W.Q.; Chen, J.; Graetz, J. Single-crystal intermetallic M-Sn (M = Fe, Cu, Co, Ni) nanospheres as negative electrodes for lithium-ion batteries. ACS Appl. Mater. Interfaces 2010, 2, 1548-1551. [CrossRef]

57. Huang, H.; Zhu, W.; Tao, X.; Xia, Y.; Yu, Z.; Fang, J.; Gan, Y.; Zhang, W. Nanocrystal-constructed mesoporous single-crystalline $\mathrm{Co}_{3} \mathrm{O}_{4}$ nanobelts with superior rate capability for advanced lithium-ion batteries. ACS Appl. Mater. Interfaces 2012, 4, 5974-5980. [CrossRef]

58. Xia, H.; Li, K.; Guo, Y.; Guo, J.; Xu, Q.; Zhang, J. CoS 2 nanodots trapped within graphitic structured N-doped carbon spheres with efficient performances for lithium storage. J. Mater. Chem. A 2018, 6, 7148-7154. [CrossRef]

59. Jin, R.; Cui, Y.; Gao, S.; Zhang, S.; Yang, L.; Li, G. CNTs@NC@CuCo $\mathrm{S}_{4}$ nanocomposites: An advanced electrode for high performance lithium-ion batteries and supercapacitors. Electrochim. Acta 2018, 273, 43-52. [CrossRef]

60. Ali, Z.; Tang, T.; Huang, X.; Wang, Y.; Asif, M.; Hou, Y. Cobalt selenide decorated carbon spheres for excellent cycling performance of sodium ion batteries. Energy Storage Mater. 2018, 13, 19-28. [CrossRef]

61. Xu, X.; Liu, J.; Hu, R.; Liu, J.; Ouyang, L.; Zhu, M. Self-Supported CoP Nanorod Arrays Grafted on Stainless Steel as an Advanced Integrated Anode for Stable and Long-Life Lithium-Ion Batteries. Chem-Eur. J. 2017, 23, 5198-5204. [CrossRef] [PubMed] 
62. Li, Q.; Wu, J.; Xu, J.; Dravid, V.P. Synergistic sodiation of cobalt oxide nanoparticles and conductive carbon nanotubes (CNTs) for sodium-ion batteries. J. Mater. Chem. A 2016, 4, 8669-8675. [CrossRef]

63. Jian, Z.; Liu, P.; Li, F.; Chen, M.; Zhou, H. Monodispersed hierarchical Co3O4spheres intertwined with carbon nanotubes for use as anode materials in sodium-ion batteries. J. Mater. Chem. A 2014, 2, 13805. [CrossRef]

64. Xiao, S.; Li, A.; Chen, X.H.; Zhou, J.S.; Ma, Z.K. Sn-Co nano alloy embedded in porous N-doped carbon microboxes as a stable anode material for lithium-ion batteries. J. Mater. Chem. A 2017, 5, 5873-5879.

65. Lou, X.W.; Deng, D.; Lee, J.Y.; Feng, J.; Archer, L.A. Self-Supported Formation of Needlelike $\mathrm{Co}_{3} \mathrm{O}_{4} \mathrm{Nannotubes}$ and Their Application as Lithium-Ion Battery Electrodes. Adv. Mater. 2008, 20, 258-262. [CrossRef]

66. Wang, Q.; Zou, R.; Xia, W.; Ma, J.; Qiu, B.; Mahmood, A.; Zhao, R.; Yang, Y.; Xia, D.; Xu, Q. Facile Synthesis of Ultrasmall $\mathrm{CoS}_{2}$ Nanoparticles within Thin N-Doped Porous Carbon Shell for High Performance Lithium-Ion Batteries. Small 2015, 11, 2511-2517. [CrossRef]

67. Gu, Y.; Wu, F.; Wang, Y. Confined Volume Change in Sn-Co-C Ternary Tube-in-Tube Composites for High-Capacity and Long-Life Lithium Storage. Adv. Funct. Mater. 2013, 23, 893-899. [CrossRef]

68. Li, J.; Yan, D.; Lu, T.; Yao, Y.; Pan, L. An advanced CoSe embedded within porous carbon polyhedra hybrid for high performance lithium-ion and sodium-ion batteries. Chem. Eng. J. 2017, 325, 14-24. [CrossRef]

69. Wang, J.; Yang, N.; Tang, H.; Dong, Z.; Jin, Q.; Yang, M.; Kisailus, D.; Zhao, H.; Tang, Z.; Wang, D. Accurate control of multishelled $\mathrm{Co}_{3} \mathrm{O}_{4}$ hollow microspheres as high-performance anode materials in lithium-ion batteries. Angew. Chem. Int. Ed. Engl. 2013, 52, 6417-6420. [CrossRef]

70. Li, Z.; Zhang, L.; Ge, X.; Li, C.; Dong, S.; Wang, C.; Yin, L. Core-shell structured CoP/FeP porous microcubes interconnected by reduced graphene oxide as high performance anodes for sodium ion batteries. Nano Energy 2017, 32, 494-502. [CrossRef]

71. Park, S.-K.; Kim, J.K.; Chan Kang, Y. Metal-organic framework-derived $\mathrm{CoSe}_{2} /(\mathrm{NiCo}) \mathrm{Se}_{2}$ box-in-box hollow nanocubes with enhanced electrochemical properties for sodium-ion storage and hydrogen evolution. J. Mater. Chem. A 2017, 5, 18823-18830. [CrossRef]

72. Alcantara, R.; Jaraba, M.; Lavela, P.; Tirado, J.L. $\mathrm{NiCo}_{2} \mathrm{O}_{4}$ Spinel: First Report on a Transition Metal Oxide for the Negative Electrode of Sodium-Ion Batteries. Chem. Mater. 2002, 14, 7. [CrossRef]

73. Du, N.; Zhang, H.; Chen, B.D.; Wu, J.B.; Ma, X.Y.; Liu, Z.H.; Zhang, Y.Q.; Yang, D.R.; Huang, X.H.; Tu, J.P. Porous $\mathrm{Co}_{3} \mathrm{O}_{4}$ Nanotubes Derived From Co4(CO)12 Clusters on Carbon Nanotube Templates: A Highly Efficient Material For Li-Battery Applications. Adv. Mater. 2007, 19, 4505-4509. [CrossRef]

74. Liu, B.; Zhang, X.; Shioyama, H.; Mukai, T.; Sakai, T.; Xu, Q. Converting cobalt oxide subunits in cobalt metal-organic framework into agglomerated $\mathrm{Co}_{3} \mathrm{O}_{4}$ nanoparticles as an electrode material for lithium ion battery. J. Power Sources 2010, 195, 857-861. [CrossRef]

75. Lu, Y.; Wang, Y.; Zou, Y.; Jiao, Z.; Zhao, B.; He, Y.; Wu, M. Macroporous $\mathrm{Co}_{3} \mathrm{O}_{4}$ platelets with excellent rate capability as anodes for lithium ion batteries. Electrochem. Commun. 2010, 12, 101-105. [CrossRef]

76. Hu, L.; Yan, N.; Chen, Q.; Zhang, P.; Zhong, H.; Zheng, X.; Li, Y.; Hu, X. Fabrication based on the Kirkendall effect of $\mathrm{Co}_{3} \mathrm{O}_{4}$ porous nanocages with extraordinarily high capacity for lithium storage. Chem-Eur. J. 2012, 18, 8971-8977. [CrossRef]

77. Yan, N.; Hu, L.; Li, Y.; Wang, Y.; Zhong, H.; Hu, X.; Kong, X.; Chen, Q. $\mathrm{Co}_{3} \mathrm{O}_{4}$ Nanocages for High-Performance Anode Material in Lithium-Ion Batteries. J. Phy. Chem. C 2012, 116, 7227-7235. [CrossRef]

78. Huang, X.L.; Wang, R.Z.; Xu, D.; Wang, Z.L.; Wang, H.G.; Xu, J.J.; Wu, Z.; Liu, Q.C.; Zhang, Y.; Zhang, X.B. Homogeneous $\mathrm{CoO}$ on Graphene for Binder-Free and Ultralong-Life Lithium Ion Batteries. Adv. Funct. Mater. 2013, 23, 4345-4353. [CrossRef]

79. Yang, D.; Zhu, J.; Rui, X.; Tan, H.; Cai, R.; Hoster, H.E.; Yu, D.Y.; Hng, H.H.; Yan, Q. Synthesis of cobalt phosphides and their application as anodes for lithium ion batteries. ACS Appl. Mater. Interfaces 2013, 5, 1093-1099. [CrossRef]

80. Zhao, W.; Ma, X.; Wang, G.; Long, X.; Li, Y.; Zhang, W.; Zhang, P. Carbon-coated $\mathrm{CoP}_{3}$ nanocomposites as anode materials for high-performance sodium-ion batteries. Appl. Surf. Sci. 2018, 445, 167-174. [CrossRef]

81. Li, J.; Le, D.B.; Ferguson, P.P.; Dahn, J.R. Lithium polyacrylate as a binder for tin-cobalt-carbon negative electrodes in lithium-ion batteries. Electrochim. Acta 2010, 55, 2991-2995. [CrossRef]

82. Zhang, Z.; Yang, J.; Nuli, Y.; Wang, B.; Xu, J. CoP $\mathrm{x}$ synthesis and lithiation by ball-milling for anode materials of lithium ion cells. Solid State Ionics 2005, 176, 693-697. [CrossRef]

83. Lavela, P.; Nacimiento, F.; Ortiz, G.F.; Tirado, J.L. Sn-Co-C composites obtained from resorcinol-formaldehyde gel as anodes in lithium-ion batteries. J. Solid State Electr. 2009, 14, 139-148. [CrossRef] 
84. González, J.R.; Nacimiento, F.; Alcántara, R.; Ortiz, G.F.; Tirado, J.L. Electrodeposited CoSn 2 on nickel open-cell foam: Advancing towards high power lithium ion and sodium ion batteries. CrystEngComm 2013, 15, 9196. [CrossRef]

85. Ke, F.S.; Huang, L.; Solomon, B.C.; Wei, G.Z.; Xue, L.-J.; Zhang, B.; Li, J.T.; Zhou, X.D.; Sun, S.G. Three-dimensional nanoarchitecture of $\mathrm{Sn}-\mathrm{Sb}-\mathrm{Co}$ alloy as an anode of lithium-ion batteries with excellent lithium storage performance. J. Mater. Chem. 2012, 22, 17511. [CrossRef]

86. Xue, L.J.; Xu, Y.F.; Huang, L.; Ke, F.S.; He, Y.; Wang, Y.X.; Wei, G.Z.; Li, J.T.; Sun, S.G. Lithium storage performance and interfacial processes of three dimensional porous $\mathrm{Sn}-\mathrm{Co}$ alloy electrodes for lithium-ion batteries. Electrochim. Acta 2011, 56, 5979-5987. [CrossRef]

87. Chou, S.L.; Wang, J.Z.; Liu, H.K.; Dou, S.X. Electrochemical deposition of porous Co3O4 nanostructured thin film for lithium-ion battery. J. Power Sources 2008, 182, 359-364. [CrossRef]

88. Liu, H.C.; Yen, S.K. Characterization of electrolytic $\mathrm{Co}_{3} \mathrm{O}_{4}$ thin films as anodes for lithium-ion batteries. J. Power Sources 2007, 166, 478-484. [CrossRef]

89. Groult, H.; El Ghallali, H.; Barhoun, A.; Briot, E.; Julien, C.M.; Lantelme, F.; Borensztjan, S. Study of Co-Sn and $\mathrm{Ni}-\mathrm{Sn}$ alloys prepared in molten chlorides and used as negative electrode in rechargeable lithium battery. Electrochim. Acta 2011, 56, 2656-2664. [CrossRef]

90. Jang, B.O.; Park, S.H.; Lee, W.J. Electrospun Co-Sn alloy/carbon nanofibers composite anode for lithium ion batteries. J. Alloy. Compd. 2013, 574, 325-330. [CrossRef]

91. Zhang, W.; Yue, Z.; Miao, W.; Liu, S.; Fu, C.; Li, L.; Zhang, Z.; Wang, H. Carbon-Encapsulated Tube-Wire $\mathrm{Co}_{3} \mathrm{O}_{4} / \mathrm{MnO}_{2}$ Heterostructure Nanofibers as Anode Material for Sodium-Ion Batteries. Part. Part. Syst. Char. 2018, 35, 1800138. [CrossRef]

92. Shin, J.; Ryu, W.-H.; Park, K.-S.; Kim, I.-D. Morphological Evolution of Carbon Nanofibers Encapsulating SnCo Alloys and Its Effect on Growth of the Solid Electrolyte Interphase Layer. ACS Nano 2013, 7, 7330-7341. [CrossRef] [PubMed]

93. Wang, J.; Wang, H.; Cao, D.; Lu, X.; Han, X.; Niu, C. Epitaxial Growth of Urchin-Like CoSe 2 Nanorods from Electrospun Co-Embedded Porous Carbon Nanofibers and Their Superior Lithium Storage Properties. Part. Part. Syst. Char. 2017, 34, 1700185. [CrossRef]

94. Qiu, H.; Wang, Y.; Liu, Y.; Li, D.; Zhu, X.; Ji, Q.; Quan, F.; Xia, Y. Synthesis of $\mathrm{Co} / \mathrm{Co}_{3} \mathrm{O}_{4}$ nanoparticles embedded in porous carbon nanofibers for high performance lithium-ion battery anodes. J. Porous. Mat. 2016, 24, 551-557. [CrossRef]

95. Chen, J.; Xia, X.H.; Tu, J.P.; Xiong, Q.Q.; Yu, Y.X.; Wang, X.L.; Gu, C.D. $\mathrm{Co}_{3} \mathrm{O}_{4}-\mathrm{C}$ core-shell nanowire array as an advanced anode material for lithium ion batteries. J. Mater. Chem. 2012, 22, 15056. [CrossRef]

96. Park, J.H.; Jeong, D.H.; Cha, S.M.; Sun, Y.K.; Yoon, C.S. Electrochemical behaviour of Heusler alloy $\mathrm{Co}_{2} \mathrm{MnSi}$ for secondary lithium batteries. J. Power Sources 2009, 188, 281-285. [CrossRef]

97. Wang, X.; Kong, D.; Huang, Z.X.; Wang, Y.; Yang, H.Y. Nontopotactic Reaction in Highly Reversible Sodium Storage of Ultrathin $\mathrm{Co}_{9} \mathrm{Se}_{8} / \mathrm{rGO}$ Hybrid Nanosheets. Small 2017, 13. [CrossRef]

98. Barreca, D.; Cruz-Yusta, M.; Gasparotto, A.; Maccato, C.; Morales, J.; Pozza, A.; Sada, C.; Sa'nchez, L.; Tondello, E. Cobalt Oxide Nanomaterials by Vapor-Phase Synthesis for Fast and Reversible Lithium Storage. J. Phys. Chem. C 2010, 114, 10054-10060. [CrossRef]

99. Jena, A.; Munichandraiah, N.; Shivashankar, S.A. Metal-organic chemical vapor-deposited cobalt oxide films as negative electrodes for thin film Li-ion battery. J. Power Sources 2015, 277, 198-204. [CrossRef]

100. Liu, H.J.; Bo, S.H.; Cui, W.J.; Li, F.; Wang, C.X.; Xia, Y.Y. Nano-sized cobalt oxide/mesoporous carbon sphere composites as negative electrode material for lithium-ion batteries. Electrochim. Acta 2008, 53, 6497-6503. [CrossRef]

101. Liao, C.L.; Lee, Y.H.; Chang, S.T.; Fung, K.Z. Structural characterization and electrochemical properties of RF-sputtered nanocrystalline $\mathrm{Co}_{3} \mathrm{O}_{4}$ thin-film anode. J. Power Sources 2006, 158, 1379-1385. [CrossRef]

102. Huang, L.; Cai, J.S.; He, Y.; Ke, F.S.; Sun, S.G. Structure and electrochemical performance of nanostructured Sn-Co alloy/carbon nanotube composites as anodes for lithium ion batteries. Electrochem. Commun. 2009, 11, 950-953. [CrossRef]

103. Li, M.Y.; Liu, C.L.; Shi, M.R.; Dong, W.S. Nanostructure Sn-Co-C composite lithium ion battery electrode with unique stability and high electrochemical performance. Electrochim. Acta 2011, 56, 3023-3028. [CrossRef] 
104. Zhai, C.; Du, N.; Zhang, H.; Yu, J.; Wu, P.; Xiao, C.; Yang, D. Assembling $\mathrm{CoSn}_{3}$ nanoparticles on multiwalled carbon nanotubes with enhanced lithium storage properties. Nanoscale 2011, 3, 1798-1801. [CrossRef] [PubMed]

105. Wang, X.L.; Chen, H.; Bai, J.; Han, W.Q. CoSn 5 Phase: Crystal Structure Resolving and Stable High Capacity as Anodes for Li Ion Batteries. J. Phys. Chem. Lett. 2012, 3, 1488-1492. [CrossRef] [PubMed]

106. Chen, P.; Guo, L.; Wang, Y. Graphene wrapped SnCo nanoparticles for high-capacity lithium ion storage. J. Power Sources 2013, 222, 526-532. [CrossRef]

107. Zhu, J.; Deng, D. Amorphous Bimetallic $\mathrm{Co}_{3} \mathrm{Sn}_{2}$ Nanoalloys Are Better Than Crystalline Counterparts for Sodium Storage. J. Phys. Chem. C 2015, 119, 21323-21328. [CrossRef]

108. Cui, W.; Wang, F.; Wang, J.; Wang, C.; Xia, Y. Nanostructural CoSnC anode prepared by $\mathrm{CoSnO}_{3}$ with improved cyclability for high-performance Li-ion batteries. Electrochim. Acta 2011, 56, 4812-4818. [CrossRef]

109. Zhao, X.; Wang, G.; Zhou, Y.; Wang, H. Flexible free-standing ternary $\mathrm{CoSnO}_{3}$ /graphene/carbon nanotubes composite papers as anodes for enhanced performance of lithium-ion batteries. Energy 2017, 118, 172-180. [CrossRef]

110. Kim, H.; Seo, D.-H.; Kim, S.-W.; Kim, J.; Kang, K. Highly reversible $\mathrm{Co}_{3} \mathrm{O}_{4}$ /graphene hybrid anode for lithium rechargeable batteries. Carbon 2011, 49, 326-332. [CrossRef]

111. Li, B.; Cao, H.; Shao, J.; Li, G.; Qu, M.; Yin, G. $\mathrm{Co}_{3} \mathrm{O}_{4} @$ graphene composites as anode materials for high-performance lithium ion batteries. Inorg. Chem. 2011, 50, 1628-1632. [CrossRef] [PubMed]

112. Jayaprakash, N.; Jones, W.D.; Moganty, S.S.; Archer, L.A. Composite lithium battery anodes based on carbon@ $\mathrm{Co}_{3} \mathrm{O}_{4}$ nanostructures: Synthesis and characterization. J. Power Sources 2012, 200, 53-58. [CrossRef]

113. Tao, L.; Zai, J.; Wang, K.; Zhang, H.; Xu, M.; Shen, J.; Su, Y.; Qian, X. $\mathrm{Co}_{3} \mathrm{O}_{4}$ nanorods/graphene nanosheets nanocomposites for lithium ion batteries with improved reversible capacity and cycle stability. J. Power Sources 2012, 202, 230-235. [CrossRef]

114. Wang, R.T.; Kong, L.B.; Lang, J.W.; Wang, X.W.; Fan, S.Q.; Luo, Y.C.; Kang, L. Mesoporous $\mathrm{Co}_{3} \mathrm{O}_{4}$ materials obtained from cobalt-citrate complex and their high capacitance behavior. J. Power Sources 2012, 217, 358-363. [CrossRef]

115. Huang, S.; Jin, Y.; Jia, M. Preparation of graphene/ $\mathrm{Co}_{3} \mathrm{O}_{4}$ composites by hydrothermal method and their electrochemical properties. Electrochim. Acta 2013, 95, 139-145. [CrossRef]

116. Rai, A.K.; Gim, J.; Anh, L.T.; Kim, J. Partially reduced $\mathrm{Co}_{3} \mathrm{O}_{4}$ /graphene nanocomposite as an anode material for secondary lithium ion battery. Electrochim. Acta 2013, 100, 63-71. [CrossRef]

117. Wen, J.W.; Zhang, D.W.; Zang, Y.; Sun, X.; Cheng, B.; Ding, C.X.; Yu, Y.; Chen, C.H. Li and Na storage behavior of bowl-like hollow $\mathrm{Co}_{3} \mathrm{O}_{4}$ microspheres as an anode material for lithium-ion and sodium-ion batteries. Electrochim. Acta 2014, 132, 193-199. [CrossRef]

118. Chen, S.; Zhao, Y.; Sun, B.; Ao, Z.; Xie, X.; Wei, Y.; Wang, G. Microwave-assisted synthesis of mesoporous $\mathrm{Co}_{3} \mathrm{O}_{4}$ nanoflakes for applications in lithium ion batteries and oxygen evolution reactions. ACS Appl. Mater. Interfaces 2015, 7, 3306-3313. [CrossRef]

119. DeliWang, Y.Y.; Huan, H.; Jie, W.; WeiDong, Z.; Hector, D. Abruna Template-Free Synthesis of Hollow-Structured $\mathrm{Co}_{3} \mathrm{O}_{4}$ Nanoparticles as High-Performance Anodes for Lithium-Ion Batteries. ACS Nano 2015, 9, 1775-1781.

120. Deng, Q.; Wang, L.; Li, J. Electrochemical characterization of $\mathrm{Co}_{3} \mathrm{O}_{4} / \mathrm{MCNTs}$ composite anode materials for sodium-ion batteries. J. Mater. Sci. 2015, 50, 4142-4148. [CrossRef]

121. Gu, D.; Li, W.; Wang, F.; Bongard, H.; Spliethoff, B.; Schmidt, W.; Weidenthaler, C.; Xia, Y.; Zhao, D.; Schuth, F. Controllable Synthesis of Mesoporous Peapod-like $\mathrm{Co}_{3} \mathrm{O}_{4} @$ Carbon Nanotube Arrays for High-Performance Lithium-Ion Batteries. Angew. Chem. Int. Ed. Engl. 2015, 54, 7060-7064. [CrossRef] [PubMed]

122. Rahman, M.M.; Sultana, I.; Chen, Z.; Srikanth, M.; Li, L.H.; Dai, X.J.; Chen, Y. Ex situ electrochemical sodiation/desodiation observation of $\mathrm{Co}_{3} \mathrm{O}_{4}$ anchored carbon nanotubes: A high performance sodium-ion battery anode produced by pulsed plasma in a liquid. Nanoscale 2015, 7, 13088-13095. [CrossRef] [PubMed]

123. Dou, Y.; Xu, J.; Ruan, B.; Liu, Q.; Pan, Y.; Sun, Z.; Dou, S.X. Atomic Layer-by-Layer $\mathrm{Co}_{3} \mathrm{O}_{4} / \mathrm{Graphene}$ Composite for High Performance Lithium-Ion Batteries. Adv. Energy Mater. 2016, 6, 1501835. [CrossRef]

124. Wang, Y.; Wang, C.; Wang, Y.; Liu, H.; Huang, Z. Superior sodium-ion storage performance of $\mathrm{Co}_{3} \mathrm{O}_{4} @$ nitrogen-doped carbon: Derived from a metal-organic framework. J. Mater. Chem. A 2016, 4, 5428-5435. [CrossRef] 
125. Yan, C.; Chen, G.; Zhou, X.; Sun, J.; Lv, C. Template-Based Engineering of Carbon-Doped $\mathrm{Co}_{3} \mathrm{O}_{4} \mathrm{Hollow}$ Nanofibers as Anode Materials for Lithium-Ion Batteries. Adv. Funct. Mater. 2016, 26, 1428-1436. [CrossRef]

126. Kaneti, Y.V.; Zhang, J.; He, Y.-B.; Wang, Z.; Tanaka, S.; Hossain, M.S.A.; Pan, Z.Z.; Xiang, B.; Yang, Q.-H.; Yamauchi, Y. Fabrication of an MOF-derived heteroatom-doped $\mathrm{Co} / \mathrm{CoO} /$ carbon hybrid with superior sodium storage performance for sodium-ion batteries. J. Mater. Chem. A 2017, 5, 15356-15366. [CrossRef]

127. Park, S.K.; Kim, J.K.; Kim, J.H.; Kang, Y.C. Metal-organic framework-templated hollow $\mathrm{Co}_{3} \mathrm{O}_{4}$ nanosphere aggregate/N-doped graphitic carbon composite powders showing excellent lithium-ion storage performances. Mater. Charact. 2017, 132, 320-329. [CrossRef]

128. Sun, Y.; Huang, F.; Li, S.; Shen, Y.; Xie, A. Novel porous starfish-like $\mathrm{Co}_{3} \mathrm{O}_{4} @$ nitrogen-doped carbon as an advanced anode for lithium-ion batteries. Nano Res. 2017, 10, 3457-3467. [CrossRef]

129. Wu, Y.; Meng, J.; Li, Q.; Niu, C.; Wang, X.; Yang, W.; Li, W.; Mai, L. Interface-modulated fabrication of hierarchical yolk-shell $\mathrm{Co}_{3} \mathrm{O}_{4} / \mathrm{C}$ dodecahedrons as stable anodes for lithium and sodium storage. Nano Res. 2017, 10, 2364-2376. [CrossRef]

130. Xin, D.; Dai, J.; Liu, J.; Wang, Q.; Li, W. Mesocrystal hexagonal $\mathrm{Co}_{3} \mathrm{O}_{4}$ nanosheets for high performance lithium and sodium-ion batteries. Mater. Lett. 2017, 209, 388-391. [CrossRef]

131. Li, J.; Li, Z.; Ning, F.; Zhou, L.; Zhang, R.; Shao, M.; Wei, M. Ultrathin Mesoporous $\mathrm{Co}_{3} \mathrm{O}_{4}$ Nanosheet Arrays for High-Performance Lithium-Ion Batteries. ACS Omega. 2018, 3, 1675-1683. [CrossRef] [PubMed]

132. Li, J.; Yan, D.; Hou, S.; Lu, T.; Yao, Y.; Pan, L. Metal-organic frameworks converted flower-like hybrid with $\mathrm{Co}_{3} \mathrm{O}_{4}$ nanoparticles decorated on nitrogen-doped carbon sheets for boosted lithium storage performance. Chem. Eng. J. 2018. [CrossRef]

133. Shao, J.; Zhou, H.; Zhu, M.; Feng, J.; Yuan, A. Facile synthesis of metal-organic framework-derived $\mathrm{Co}_{3} \mathrm{O}_{4}$ with different morphologies coated graphene foam as integrated anodes for lithium-ion batteries. J. Alloy. Compd. 2018, 768, 1049-1057. [CrossRef]

134. Kwon, H.T.; Kim, J.H.; Jeon, K.J.; Park, C.M. $\mathrm{Co}_{\mathrm{x}} \mathrm{P}$ compounds: Electrochemical conversion/partial recombination reaction and partially disproportionated nanocomposite for Li-ion battery anodes. RSC Adv. 2014, 4, 43227-43234. [CrossRef]

135. Yang, J.; Zhang, Y.; Sun, C.; Liu, H.; Li, L.; Si, W.; Huang, W.; Yan, Q.; Dong, X. Graphene and cobalt phosphide nanowire composite as an anode material for high performance lithium-ion batteries. Nano Res. 2016, 9, 612-621. [CrossRef]

136. Guo, Q.; Ru, Q.; Wang, B.; Mo, Y.; Wang, Z.; Zhang, P.; Hou, X.; Hu, S. The electrochemical confrontation between $\mathrm{CoP}$ microflake and $\mathrm{Co}_{3} \mathrm{O}_{4}$ microsphere via a similar synthesis process as anodes for lithium ion batteries. J. Alloy. Compd. 2017, 728, 910-916. [CrossRef]

137. Guo, Q.; Ru, Q.; Wang, B.; Zhang, P.; Hou, X.; Ling, F.C.-C. Design and Synthesis of Mesoporous Honeycomb-Like $\mathrm{CoP} / \mathrm{Co}_{2} \mathrm{P}$ Hybrids as Anode with a High Cyclic Stability in Lithium-Ion Batteries. Energy Technol. 2017, 5, 2294-2299. [CrossRef]

138. Wang, B.; Ru, Q.; Guo, Q.; Chen, X.; Wang, Z.; Hou, X.; Hu, S. Fabrication of One-Dimensional Mesoporous CoP Nanorods as Anode Materials for Lithium-Ion Batteries. Eur. J. Inorg. Chem. 2017, 2017, 3729-3735. [CrossRef]

139. Xie, Q.; Zeng, D.; Gong, P.; Huang, J.; Ma, Y.; Wang, L.; Peng, D.-L. One-pot fabrication of graphene sheets decorated $\mathrm{Co}_{2} \mathrm{P}-\mathrm{Co}$ hollow nanospheres for advanced lithium ion battery anodes. Electrochim. Acta 2017, 232, 465-473. [CrossRef]

140. Xing, Y.M.; Zhang, X.H.; Liu, D.H.; Li, W.H.; Sun, L.N.; Geng, H.B.; Zhang, J.P.; Guan, H.Y.; Wu, X.L. Porous Amorphous $\mathrm{Co}_{2} \mathrm{P} / \mathrm{N}, \mathrm{B}-\mathrm{Co}$-doped Carbon Composite as an Improved Anode Material for Sodium-Ion Batteries. ChemElectroChem 2017, 4, 1395-1401. [CrossRef]

141. Bai, J.; Xi, B.; Mao, H.; Lin, Y.; Ma, X.; Feng, J.; Xiong, S. One-Step Construction of N,P-Codoped Porous Carbon Sheets/CoP Hybrids with Enhanced Lithium and Potassium Storage. Adv. Mater. 2018, e1802310. [CrossRef] [PubMed]

142. Jiang, J.; Zhu, K.; Fang, Y.; Wang, H.; Ye, K.; Yan, J.; Wang, G.; Cheng, K.; Zhou, L.; Cao, D. Coralloidal carbon-encapsulated CoP nanoparticles generated on biomass carbon as a high-rate and stable electrode material for lithium-ion batteries. J. Colloid Interface Sci. 2018, 530, 579-585. [CrossRef] [PubMed]

143. Jin, R.; Li, X.; Sun, Y.; Shan, H.; Fan, L.; Li, D.; Sun, X. Metal-Organic Frameworks-Derived $\mathrm{Co}_{2} \mathrm{P} @ \mathrm{~N}-\mathrm{C} @ \mathrm{rGO}$ with Dual Protection Layers for Improved Sodium Storage. ACS Appl. Mater. Interfaces 2018, 10, 14641-14648. [CrossRef] [PubMed] 
144. Liu, Q.; Luo, Y.; Chen, W.; Yan, Y.; Xue, L.; Zhang, W. $\mathrm{CoP}_{3} @ P P y$ microcubes as anode for lithium-ion batteries with improved cycling and rate performance. Chem. Eng. J. 2018, 347, 455-461. [CrossRef]

145. Zhang, L.; Li, H.; Xie, H.; Chen, T.; Yang, C.; Wang, J. MOF-driven ultra-small hollow CogS 8 nanoparticles embedded in porous carbon for lithium-ion batteries. J. Mater. Res. 2018, 33, 1496-1505. [CrossRef]

146. Yu, J.; Li, X.; Sun, Y.; Liu, X. CoS@sulfur doped onion-like carbon nanocapsules with excellent cycling stability and rate capability for sodium-ion batteries. Ceram. Int. 2018, 44, 17113-17117. [CrossRef]

147. Wang, X.; Li, X.; Li, Q.; Li, H.; Xu, J.; Wang, H.; Zhao, G.; Lu, L.; Lin, X.; Li, H.; et al. Improved Electrochemical Performance Based on Nanostructured $\mathrm{SnS}_{2} @ \mathrm{CoS}_{2}-\mathrm{rGO}$ Composite Anode for Sodium-Ion Batteries. Nano-Micro Lett. 2018, 10. [CrossRef]

148. Wang, H.C.; Cui, Z.; Fan, C.Y.; Liu, S.Y.; Shi, Y.H.; Wu, X.L.; Zhang, J.P. 3 D Porous $\mathrm{CoS}_{2}$ Hexadecahedron Derived from MOC toward Ultrafast and Long-Lifespan Lithium Storage. Chem-Eur. J. 2018, 24, 6798-6803. [CrossRef]

149. Liu, X.; Ma, H.; Liu, Q.; Wang, Y.; Lu, Z.; Li, M.; Wang, J.; Wang, D. Ethylenediamine-assisted synthesis of microsized cobalt sulfide as advanced anode materials for sodium ion batteries. J. Alloy. Compd. 2018, 735, 765-772. [CrossRef]

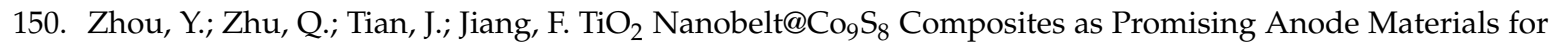
Lithium and Sodium Ion Batteries. Nanomaterials 2017, 7, 252. [CrossRef]

151. Zhou, L.; Zhang, K.; Sheng, J.; An, Q.; Tao, Z.; Kang, Y.M.; Chen, J.; Mai, L. Structural and chemical synergistic effect of $\mathrm{CoS}$ nanoparticles and porous carbon nanorods for high-performance sodium storage. Nano Energy 2017, 35, 281-289. [CrossRef]

152. Zhou, H.; Hu, J. Facile synthesis of multi-walled carbon nanotubes/Cog $\mathrm{S}_{8}$ composites with enhanced performances for sodium-ion battery. Mater. Lett. 2017, 195, 26-30. [CrossRef]

153. Zhao, Y.; Pang, Q.; Wei, Y.; Wei, L.; Ju, Y.; Zou, B.; Gao, Y.; Chen, G. CogS 8 /Co as a High-Performance Anode for Sodium-Ion Batteries with an Ether-Based Electrolyte. ChemSusChem 2017, 10, 4778-4785. [CrossRef] [PubMed]

154. Zhang, X.; Wang, H.; Wang, G. Cobalt sulfide nanoparticles anchored in three-dimensional carbon nanosheet networks for lithium and sodium ion batteries with enhanced electrochemical performance. J. Colloid Interface Sci. 2017, 492, 41-50. [CrossRef] [PubMed]

155. Zhang, J.; Yu, L.; Lou, X.W.D. Embedding $\operatorname{CoS}_{2}$ nanoparticles in N-doped carbon nanotube hollow frameworks for enhanced lithium storage properties. Nano Res. 2017, 10, 4298-4304. [CrossRef]

156. Tao, S.; Huang, W.; Xie, H.; Zhang, J.; Wang, Z.; Chu, W.; Qian, B.; Song, L. Formation of graphene-encapsulated $\mathrm{CoS}_{2}$ hybrid composites with hierarchical structures for high-performance lithium-ion batteries. RSC Adv. 2017, 7, 39427-39433. [CrossRef]

157. Pan, Y.; Cheng, X.; Huang, Y.; Gong, L.; Zhang, H. $\mathrm{CoS}_{2}$ Nanoparticles Wrapping on Flexible Freestanding Multichannel Carbon Nanofibers with High Performance for Na-Ion Batteries. ACS Appl. Mater. Interfaces 2017, 9, 35820-35828. [CrossRef]

158. Liu, X.; Zou, F.; Liu, K.; Qiang, Z.; Taubert, C.J.; Ustriyana, P.; Vogt, B.D.; Zhu, Y. A binary metal organic framework derived hierarchical hollow $\mathrm{Ni}_{3} \mathrm{~S}_{2} / \mathrm{Cog}_{8} \mathrm{~S}_{8} / \mathrm{N}$-doped carbon composite with superior sodium storage performance. J. Mater. Chem. A 2017, 5, 11781-11787. [CrossRef]

159. Zhou, Y.; Tian, R.; Duan, H.; Wang, K.; Guo, Y.; Li, H.; Liu, H. CoSe/Co nanoparticles wrapped by in situ grown N-doped graphitic carbon nanosheets as anode material for advanced lithium ion batteries. J. Power Sources 2018, 399, 223-230. [CrossRef]

160. Zhang, G.; Liu, K.; Liu, S.; Song, H.; Zhou, J. Flexible $\mathrm{Co}_{0.85}$ Se nanosheets/graphene composite film as binder-free anode with high Li- and Na-Ion storage performance. J. Alloy. Compd. 2018, 731, 714-722. [CrossRef]

161. Sun, W.; Cai, C.; Tang, X.; Lv, L.-P.; Wang, Y. Carbon coated mixed-metal selenide microrod: Bimetal-organic-framework derivation approach and applications for lithium-ion batteries. Chem. Eng. J. 2018, 351, 169-176. [CrossRef]

162. Park, S.K.; Kang, Y.C. MOF-Templated N-Doped Carbon-Coated $\mathrm{CoSe}_{2}$ Nanorods Supported on Porous CNT Microspheres with Excellent Sodium-Ion Storage and Electrocatalytic Properties. ACS Appl. Mater. Interfaces 2018, 10, 17203-17213. [CrossRef] [PubMed]

163. Fang, Y.; Yu, X.Y.; Lou, X.W.D. Formation of Hierarchical Cu-Doped $\mathrm{CoSe}_{2}$ Microboxes via Sequential Ion Exchange for High-Performance Sodium-Ion Batteries. Adv. Mater. 2018, 30, e1706668. [CrossRef] [PubMed] 
164. Cai, T.; Zhao, L.; Hu, H.; Li, T.; Li, X.; Guo, S.; Li, Y.; Xue, Q.; Xing, W.; Yan, Z.; et al. Stable CoSe $2 /$ carbon nanodice@reduced graphene oxide composites for high-performance rechargeable aluminum-ion batteries. Energy Environ. Sci. 2018, 11, 2341-2347. [CrossRef]

165. Zhu, Y.; Chen, X.; Chen, H.; Ji, X.; Liu, Y. 3D Nanosheet-Assembled CoSe Quasi-Microspheres as Advanced Electrode Materials for Electrochemical Energy Storage. J. Electrochem. Soc. 2017, 164, A2341-A2347. [CrossRef]

166. Zhang, Y.; Pan, A.; Ding, L.; Zhou, Z.; Wang, Y.; Niu, S.; Liang, S.; Cao, G. Nitrogen-Doped Yolk-Shell-Structured CoSe/C Dodecahedra for High-Performance Sodium Ion Batteries. ACS Appl. Mater. Interfaces 2017, 9, 3624-3633. [CrossRef]

167. Park, S.-K.; Kim, J.K.; Kang, Y.C. Excellent sodium-ion storage performances of $\mathrm{CoSe}_{2}$ nanoparticles embedded within $\mathrm{N}$-doped porous graphitic carbon nanocube/carbon nanotube composite. Chem. Eng. J. 2017, 328, 546-555. [CrossRef]

168. Ou, X.; Liang, X.; Zheng, F.; Wu, P.; Pan, Q.; Xiong, X.; Yang, C.; Liu, M. In situ X-ray diffraction investigation of $\mathrm{CoSe}_{2}$ anode for Na-ion storage: Effect of cut-off voltage on cycling stability. Electrochim. Acta 2017, 258, 1387-1396. [CrossRef]

169. Lu, W. CoSe 2 Nanoparticles as Anode for Lithium Ion Battery. Int. J. Electrochem. Sci. 2017, 12, 1118-1129. [CrossRef]

170. Liu, X.; Liu, Y.; Fan, L.-Z. MOF-derived CoSe 2 microspheres with hollow interiors as high-performance electrocatalysts for the enhanced oxygen evolution reaction. J. Mater. Chem. A 2017, 5, 15310-15314. [CrossRef]

171. Qiu, S.; Huang, J.; Chu, H.; Zou, Y.; Xiang, C.; Yan, E.; Xu, F.; Sun, L. The Co-B Amorphous Alloy: A High Capacity Anode Material for an Alkaline Rechargeable Battery. Metals 2016, 6, 269. [CrossRef]

172. Chen, Z.; Wu, R.; Liu, M.; Wang, H.; Xu, H.; Guo, Y.; Song, Y.; Fang, F.; Yu, X.; Sun, D. General Synthesis of Dual Carbon-Confined Metal Sulfides Quantum Dots Toward High-Performance Anodes for Sodium-Ion Batteries. Adv. Funct. Mater. 2017, 27, 1702046. [CrossRef]

173. Peng, S.; Han, X.; Li, L.; Zhu, Z.; Cheng, F.; Srinivansan, M.; Adams, S.; Ramakrishna, S. Unique Cobalt Sulfide/Reduced Graphene Oxide Composite as an Anode for Sodium-Ion Batteries with Superior Rate Capability and Long Cycling Stability. Small 2016, 12, 1359-1368. [CrossRef] [PubMed]

174. Yu, L.; Yang, J.F.; Lou, X.W. Formation of $\mathrm{CoS}_{2}$ Nanobubble Hollow Prisms for Highly Reversible Lithium Storage. Angew. Chem. Int. Ed. Engl. 2016, 55, 13422-13426. [CrossRef] [PubMed]

175. Zhou, Y.; Yan, D.; Xu, H.; Feng, J.; Jiang, X.; Yue, J.; Yang, J.; Qian, Y. Hollow nanospheres of mesoporous $\mathrm{Co}_{9} \mathrm{~S}_{8}$ as a high-capacity and long-life anode for advanced lithium ion batteries. Nano Energy 2015, 12, 528-537. [CrossRef]

176. Su, Q.; Du, G.; Zhang, J.; Zhong, Y.; Xu, B.; Yang, Y.; Li, W. In Situ Transmission Electron Microscopy Observation of Electrochemical Sodiation of Individual Co9S8-Filled Carbon Nanotubes. ACS Nano 2014, 8, 3620-3627. [CrossRef]

177. Jin, R.; Liu, J.; Xu, Y.; Li, G.; Chen, G. Solvothermal synthesis and excellent electrochemical performance of polycrystalline rose-like $\mathrm{Co}_{9} \mathrm{~S}_{8}$ hierarchical architectures. J. Mater. Chem. A 2013, 1, 7995. [CrossRef]

178. Qiu, B.; Zhao, X.; Xia, D. In situ synthesis of $\mathrm{CoS}_{2} /$ RGO nanocomposites with enhanced electrode performance for lithium-ion batteries. J. Alloy. Compd. 2013, 579, 372-376. [CrossRef]

179. Su, Q.; Xie, J.; Zhang, J.; Zhong, Y.; Du, G.; Xu, B. In situ transmission electron microscopy observation of electrochemical behavior of $\mathrm{CoS}_{2}$ in lithium-ion battery. ACS Appl. Mater. Interfaces 2014, 6, 3016-3022. [CrossRef]

180. Jin, R.; Yang, L.; Li, G.; Chen, G. Hierarchical worm-like $\mathrm{CoS}_{2}$ composed of ultrathin nanosheets as an anode material for lithium-ion batteries. J. Mater. Chem. A 2015, 3, 10677-10680. [CrossRef]

181. Zhang, Z.; Gan, Y.; Lai, Y.; Shi, X.; Chen, W.; Li, J. Cobalt sulfides/dodecahedral porous carbon as anode materials for Na-ion batteries. RSC Adv. 2015, 5, 103410-103413. [CrossRef]

182. Liu, J.; Wu, C.; Xiao, D.; Kopold, P.; Gu, L.; van Aken, P.A.; Maier, J.; Yu, Y. MOF-Derived Hollow Cog $\mathrm{S}_{8}$ Nanoparticles Embedded in Graphitic Carbon Nanocages with Superior Li-Ion Storage. Small 2016, 12, 2354-2364. [CrossRef] [PubMed]

183. Liu, X.; Liu, H.; Zhao, Y.; Dong, Y.; Fan, Q.; Kuang, Q. Synthesis of the Carbon-Coated Nanoparticle Cog $\mathrm{S}_{8}$ and Its Electrochemical Performance as an Anode Material for Sodium-Ion Batteries. Langmuir 2016, 32, 12593-12602. [CrossRef] [PubMed] 
184. Liu, X.; Zhang, K.; Lei, K.; Li, F.; Tao, Z.; Chen, J. Facile synthesis and electrochemical sodium storage of $\mathrm{CoS}_{2}$ micro/nano-structures. Nano Res. 2016, 9, 198-206. [CrossRef]

185. Meng, X.; Deng, D. Trash to Treasure: Waste Eggshells Used as Reactor and Template for Synthesis of Cog $\mathrm{S}_{8}$ Nanorod Arrays on Carbon Fibers for Energy Storage. Chem. Mater. 2016, 28, 3897-3904. [CrossRef]

186. Guo, Q.; Ma, Y.; Chen, T.; Xia, Q.; Yang, M.; Xia, H.; Yu, Y. Cobalt Sulfide Quantum Dot Embedded N/S-Doped Carbon Nanosheets with Superior Reversibility and Rate Capability for Sodium-Ion Batteries. ACS Nano 2017, 11, 12658-12667. [CrossRef]

187. He, J.; Chen, Y.; Li, P.; Fu, F.; Wang, Z.; Zhang, W. Self-assembled $\mathrm{CoS}_{2}$ nanoparticles wrapped by $\mathrm{CoS}_{2}$-quantum-dots-anchored graphene nanosheets as superior-capability anode for lithium-ion batteries. Electrochim. Acta 2015, 182, 424-429. [CrossRef]

188. Khatib, R.; Dalverny, A.L.; Saubanère, M.; Gaberscek, M.; Doublet, M.L. Origin of the Voltage Hysteresis in the CoP Conversion Material for Li-Ion Batteries. J. Phys. Chem. C 2013, 117, 837-849. [CrossRef]

189. Liu, L.; Li, Q.; Wang, Z.; Yan, J.; Chen, Y. Progress of Metal-Phosphide Electrodes for Advanced Sodium-Ion Batteries. Funct. Mater. Lett. 2018, 11, 1830001. [CrossRef]

190. Zhang, K.; Park, M.; Zhou, L.; Lee, G.-H.; Li, W.; Kang, Y.-M.; Chen, J. Urchin-Like CoSe 2 as a High-Performance Anode Material for Sodium-Ion Batteries. Adv. Funct. Mater. 2016, 26, 6728-6735. [CrossRef]

191. Cho, J.S.; Won, J.M.; Lee, J.-K.; Kang, Y.C. Design and synthesis of multiroom-structured metal compounds-carbon hybrid microspheres as anode materials for rechargeable batteries. Nano Energy 2016, 26, 466-478. [CrossRef]

192. Hu, H.; Zhang, J.; Guan, B.; Lou, X.W. Unusual Formation of CoSe@carbon Nanoboxes, which have an Inhomogeneous Shell, for Efficient Lithium Storage. Angew. Chem. Int. Ed. Engl. 2016, 55, 9514-9518. [CrossRef] [PubMed]

193. Ko, Y.N.; Choi, S.H.; Kang, Y.C. Hollow Cobalt Selenide Microspheres: Synthesis and Application as Anode Materials for Na-Ion Batteries. ACS Appl. Mater. Interfaces 2016, 8, 6449-6456. [CrossRef] [PubMed]

194. Zhou, J.; Wang, Y.; Zhang, J.; Chen, T.; Song, H.; Yang, H.Y. Two dimensional layered $\mathrm{Co}_{0.85}$ Se nanosheets as a high-capacity anode for lithium-ion batteries. Nanoscale 2016, 8, 14992-15000. [CrossRef] [PubMed]

195. Chen, D.; Chen, G.; Pei, J.; Hu, Y.; Qin, Z.; Wang, J.; Wu, F. Formation of Porous Cu-Doped CoSe 2 Connected by Nanoparticles for Efficient Lithium Storage. ChemElectroChem 2017, 4, 2158-2163. [CrossRef] 